\section{Population and vital statistics}

This section begins with a summary of population figures for the UK and constituent countries for 1851 to 2031 and for Great Britain from 1801 (Table 5.1). Table 5.2 analyses the components of population change. Table 5.3 gives details of the national sex and age structures for years up to the present date, with projected figures up to the year 2026. Legal marital condition of the population is shown in Table 5.4. The distribution of population at regional and local levels is summarised in Table 5.5.

In the main, historical series relate to census information, while mid-year estimates, which make allowance for underenumeration in the census, are given for the recent past and the present (from 1961 onwards).

\section{Population}

\section{(Tables $5.1-5.3$ )}

Figures shown in these tables relate to the population enumerated at successive censuses (up to 1951), mid-year estimates (from 1961 to 2008) and population projections (up to 2031). Further information can be found on the Office for National Statistics (ONS) website at: www.statistics.gov.uk/popest

Population projections are 2008-based and were published by ONS on 21 October 2009. Further information can be found at: www.statistics.gov.uk/StatBase/Product.asp?vlnk=8519.

\section{Definition of resident population}

The estimated resident population of an area includes all the people who usually live there, whatever their nationality. Members of HM and US Armed Forces in England and Wales are included on a residential basis wherever possible. HM Forces stationed outside England and Wales are not included. Students are taken to be resident at their term-time address.

The projections of the resident population of the UK and constituent countries were prepared by the National Statistics Centre for Demography within ONS, in consultation with the Registrars General, as a common framework for use in national planning in a number of different fields. New projections are made every second year on assumptions regarding future fertility, mortality and migration which seem most appropriate on the basis of the statistical evidence available at the time. The population projections in
Tables $5.1-5.3$ are based on the estimates of the population of the UK at mid-2008 made by the Registrars General.

\section{Marital condition (de jure): estimated population}

\section{(Table 5.4)}

This table shows population estimates by marital status.

\section{Geographical distribution of the population}

\section{(Table 5.5)}

The population enumerated in the censuses for 1911-1951 and the mid-year population estimates for later years are provided for standard regions of the UK, for metropolitan areas, for broad groupings of local authority districts by type within England and Wales and for some of the larger cities. Projections of future sub-national population levels are prepared from time to time by the Registrar General, but are not shown in this publication.

\section{Migration into and out of the UK}

\section{(Tables 5.7, 5.9)}

A migrant is defined as a person who changes his or her country of usual residence for a period of at least a year so that the country of destination effectively becomes the country of usual residence.

The main source of international migration data is the International Passenger Survey (IPS). This is a continuous voluntary sample survey that provides information on passengers entering and leaving the UK by the principal air, sea and tunnel routes. Being a sample survey, the IPS is subject to some uncertainty; therefore, it should be noted that international migration estimates, in particular the difference between inflow and outflow, may be subject to large sampling errors. The IPS excludes routes between the Channel Islands and Isle of Man and the rest of the world.

The IPS data are supplemented with three types of additional information in order to provide a full picture of total international migration:

1. The IPS is based on intentions to migrate and intentions are liable to change. Adjustments are made for visitor switchers (those who intend to stay in the UK or abroad for less than one year but subsequently stay for longer and become migrants) and for migrant switchers (those who intend to stay in the UK or abroad for one year or more but then return earlier so are no longer migrants). These adjustments are primarily based on IPS data but 
for years prior to 2001 Home Office data on short-term visitors who were subsequently granted an extension of stay for a year or longer for other reasons have been incorporated.

2. Home Office data on applications for asylum and dependants of asylum seekers entering the UK are used to estimate inflows of asylum seekers and dependants not already captured by the IPS. In addition, Home Office data on removals and refusals are used to estimate outflows of failed asylum seekers not identified by the IPS.

3. Migration flows between the UK and the Irish Republic are added to these data as the IPS did not cover this route until recently and the quality of these data are still being assessed. Migration flows are obtained mainly from the Quarterly National Household Survey and are agreed between the Irish Central Statistics Office and ONS.

The international migration estimates in Table 5.7 are derived from all these sources and represent total international migration. The estimates in Tables 5.8 and 5.9 are based on the IPS only (without the three adjustments outlined above).

\section{Grants for settlement in the UK}

\section{(Table 5.10)}

This table presents, in geographic regions, the statistics of individual countries of nationality, arranged alphabetically within each region. The figures are on a different basis from those derived from IPS (Tables 5.8 and 5.9) and relate only to people subject to immigration control. Persons granted settlement are allowed to stay indefinitely in the UK. They exclude temporary migrants such as students and generally relate only to non-EEA nationals. Settlement can occur several years after entry to the country.

\section{Applications received for asylum in the United Kingdom, excluding dependants}

\section{(Table 5.11)}

This table shows statistics of applications for asylum in the UK. Figures are shown for the main applicant nationalities by geographic region. The basis of assessing asylum applications, and hence of deciding whether to grant asylum in the UK, is the 1951 United Nations Convention on Refugees.

\section{Marriages}

\section{(Table 5.12)}

The figures in this table relate to marriages solemnised in the constituent countries of the UK. They take no account of the growing trend towards marrying abroad.

\section{Divorces}

(Tables 5.13 and 5.14)

A marriage may be either dissolved, following a petition for divorce and the granting of a decree absolute, or annulled, following a petition for nullity and the awarding of a decree of nullity. The first group of decrees are known as dissolutions of marriage and the second as annulments of marriage. In Table 5.13 the term 'divorce' includes both types of decrees.

\section{Births}

(Tables $5.15-5.17$ )

For Scotland and Northern Ireland the number of births relate to those registered during the year. For England and Wales the figures up to and including the period from 1930 to 1932 are for those registered, while later figures relate to births occurring in each year.

All data for England and Wales and for Scotland include births occurring in those countries to mothers not usually resident in them. Data for Northern Ireland, and hence the UK, prior to 1981 include births occurring in Northern Ireland to nonresident mothers; from 1981 such births are excluded.

\section{Deaths}

(Tables 5.19 and 5.21)

The figures relate to the number of deaths registered during each calendar year.

\section{Infant and maternal mortality}

\section{(Table 5.20)}

On 1 October 1992 the legal definition of a stillbirth was altered from a baby born dead after 28 completed weeks gestation or more to one born after 24 completed weeks of gestation or more. The 258 stillbirths of 24 to 27 weeks gestation that occurred between 1 October and 31 December 1992 are excluded from this table. 


\section{Life tables}

\section{(Table 5.22)}

The current set of interim life tables are constructed from the estimated population in the period from 2004 to 2006 and corresponding data on births, infant deaths and deaths by individual age occurring in those years.

The estimates used in these interim life tables are the estimates, or revised estimates, issued on the following dates:

$\begin{array}{lllll}\begin{array}{l}\text { Mid-year } \\ \text { population } \\ \text { estimates }\end{array} & \text { England } & \text { Wales } & \text { Scotland } & \begin{array}{l}\text { Northern } \\ \text { Ireland }\end{array} \\ 2004 & \text { August } & \text { August } & \text { July } & \text { July } \\ & 2007 & 2007 & 2007 & 2005 \\ 2005 & \text { August } & \text { August } & \text { July } & \text { October } \\ & 2007 & 2007 & 2007 & 2006 \\ 2006 & \text { August } & \text { August } & \text { July } & \text { July } \\ & 2007 & 2007 & 2007 & 2007 \\ 2007 & \text { August } & \text { August } & \text { July } & \text { July } \\ & 2008 & 2008 & 2008 & 2008\end{array}$

\section{Adoptions}

\section{(Tables 5.23)}

The figures shown within these tables relate to the date the adoption was entered in the Adopted Children Register. Figures based on the date of the court order are available for England and Wales in the volume Marriage, divorce and adoption statistics 2007 (no. 35 in the FM2 series) available on the ONS website at: www.ons.gov.uk or from the enquiry point in the ONS shown at the foot of the tables. 
Thousands

Persons Males Females Persons Males Females Persons Persons Males Females Persons Males Females

Enumerated population:

Census figures

1801
1851
1901
1911
$1921^{1}$
$1931^{1}$
1951
1961

Resident population:

mid-year estimates

\begin{tabular}{l}
1973 \\
1974 \\
1975 \\
1976 \\
1977 \\
\\
1978 \\
1979 \\
1980 \\
1981 \\
1982 \\
1983 \\
1984 \\
1985 \\
1986 \\
1987 \\
\\
1988 \\
1989 \\
1990 \\
1991 \\
1992 \\
\\
1993 \\
1994 \\
1995 \\
1996 \\
1997 \\
1998 \\
1999 \\
2000 \\
2001 \\
2002 \\
\\
2003 \\
2004 \\
2005 \\
2006 \\
2007 \\
\\
2008 \\
\hline
\end{tabular}

$\begin{array}{rrrrr}\text { DYAY } & \text { BBAB } & \text { BBAC } & \text { BBAD } & \text { BBAE } \\ 56223 & 27332 & 28891 & 49459 & 24061 \\ 56236 & 27349 & 28887 & 49468 & 24075 \\ 56226 & 27361 & 28865 & 49470 & 24091 \\ 56216 & 27360 & 28856 & 49459 & 24089 \\ 56190 & 27345 & 28845 & 49440 & 24076 \\ & & & & \\ 56178 & 27330 & 28849 & 49443 & 24067 \\ 56240 & 27373 & 28867 & 49508 & 24113 \\ 56330 & 27411 & 28919 & 49603 & 24156 \\ 56357 & 27412 & 28946 & 49634 & 24160 \\ 56291 & 27364 & 28927 & 49582 & 24119 \\ & & & & \\ 56316 & 27371 & 28944 & 49617 & 24133 \\ 56409 & 27421 & 28989 & 49713 & 24185 \\ 56554 & 27489 & 29065 & 49861 & 24254 \\ 56684 & 27542 & 29142 & 49999 & 24311 \\ 56804 & 27599 & 29205 & 50123 & 24371 \\ & & & & \\ 56916 & 27652 & 29265 & 50254 & 24434 \\ 57076 & 27729 & 29348 & 50408 & 24510 \\ 57237 & 27819 & 29419 & 50561 & 24597 \\ 57439 & 27909 & 29530 & 50748 & 24681 \\ 57585 & 27977 & 29608 & 50876 & 24739 \\ & & & & \\ 57714 & 28039 & 29675 & 50986 & 24793 \\ 57862 & 28108 & 29754 & 51116 & 24853 \\ 58025 & 28204 & 29821 & 51272 & 24946 \\ 58164 & 28287 & 29877 & 51410 & 25030 \\ 58314 & 28371 & 29943 & 51560 & 25113 \\ 58475 & 28458 & 30017 & 51720 & 25201 \\ 58684 & 28578 & 30106 & 51933 & 25323 \\ 58886 & 28690 & 30196 & 52140 & 25438 \\ 59113 & 28832 & 30281 & 52360 & 25574 \\ 59323 & 28964 & 30359 & 52572 & 25704 \\ & & & & \\ 59557 & 29109 & 30449 & 52797 & 25841 \\ 59846 & 29278 & 30568 & 53057 & 25995 \\ 60238 & 29497 & 30741 & 53419 & 26197 \\ 60587 & 29694 & 30893 & 53729 & 26371 \\ 60975 & 29916 & 31059 & 54072 & 26569 \\ & & & & \\ 61383 & 30151 & 31232 & 54440 & 26780\end{array}$

BBAF

$25393 \quad 2785$

25378

25370

25364

25375

25395

25448

25474

25462

25484

25528

25606

25687

25752

25820

25898

25964

26067

26136

26193

26263

26326

26381

26446

26519

26610

26702

26786

26868

26956

27062

27223

27358

27503

27659

2008

$61383 \quad 30151$

2803

2841

2887

2889

2891

$\begin{array}{rrr}587 & 1608 & 739 \\ 1163 & 2889 & 1376 \\ 2013 & 4472 & 2174 \\ 2421 & 4761 & 2309 \\ 2656 & 4882 & 2348 \\ & & \\ 2593 & 4843 & 2326 \\ 2599 & 5096 & 2434 \\ 2644 & 5179 & 2483\end{array}$

869

$1513 \quad 1442 \quad 698$

$2298 \quad 1237$

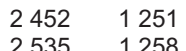

$2517 \quad 1243$

$2662 \quad 1371$

$2697 \quad 1425$
590

603
610

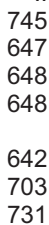

745

648

648

601

668
694

$\begin{array}{lll}2799 & 5233 & 2517\end{array}$

$2801 \quad 5226 \quad 2515$

$2804 \quad 5212 \quad 2509$

$2810 \quad 5204 \quad 2505$

$2816 \quad 5194 \quad 2501$

$2813 \quad 5180 \quad 2495$

$2804 \quad 5165 \quad 2487$

$\begin{array}{lll}5148 & 2479\end{array}$

$2801 \quad 5139 \quad 2475$

$5128 \quad 2470$

$\begin{array}{lll}2823 & 5099 & 2455\end{array}$

$5077 \quad 2444$

$2855 \quad 5078 \quad 2443$

$2862 \quad 5081 \quad 2444$

$2873 \quad 5083 \quad 2445$

$2878 \quad 5086 \quad 2445$

$2884 \quad 5092 \quad 2448$

$5102 \quad 2453$

$\begin{array}{lll}5104 & 2453\end{array}$

$\begin{array}{ll}5 & 092 \\ 5 & 447\end{array}$

BBAI

BBAJ BBAK BBAL

$\begin{array}{lll}1530 & 756 & 774\end{array}$

$\begin{array}{llll}2722 & 1527 & 755 & 772\end{array}$

$\begin{array}{llll}2716 & 1524 & 753 & 770\end{array}$

$\begin{array}{llll}2716 & 1524 & 754 & 770 \\ 2711 & 1523 & 754 & 769\end{array}$

$\begin{array}{llll}2704 & 1523 & 754 & 770\end{array}$

$\begin{array}{llll}2699 & 1528 & 755 & 773\end{array}$

$\begin{array}{llll}2693 & 1533 & 755 & 778\end{array}$

$2685 \quad 1543 \quad 757 \quad 786$

$2677 \quad 1545 \quad 757 \quad 788$

$\begin{array}{llll}2669 & 1551 & 759 & 792\end{array}$

$2664 \quad 1557 \quad 761 \quad 796$

$2658 \quad 1565 \quad 765 \quad 800$

$\begin{array}{llll}2649 & 1574 & 768 & 805\end{array}$

$2644 \quad 1582 \quad 773 \quad 809$

$2633 \quad 1585 \quad 774 \quad 812$

$2635 \quad 1590 \quad 776 \quad 814$

$\begin{array}{llll}2637 & 1596 & 778 & 818\end{array}$

$2639 \quad 1607 \quad 783 \quad 824$

$2640 \quad 1623 \quad 792 \quad 831$

$\begin{array}{llll}2644 & 1636 & 798 & 837\end{array}$

$2649 \quad 1644 \quad 802 \quad 842$

$\begin{array}{llll}2650 & 1649 & 804 & 845\end{array}$

$2645 \quad 1662 \quad 810 \quad 851$

$2641-1671 \quad 816 \quad 856$

$2900 \quad 5077 \quad 2439$

$2901 \quad 5072 \quad 2437$

$\begin{array}{llll}2638 & 1678 & 819 & 859\end{array}$

$\begin{array}{llllll}2910 & 5064 & 2434 & 2630 & 1689 & 824\end{array}$

$2920 \quad 5055 \quad 2432$

2623

$1697 \quad 829$

$2931 \quad 5057 \quad 2435$

$2946 \quad 5078 \quad 2446$

$2954 \quad 5095 \quad 2456$

$2966 \quad 5117 \quad 2469$

$2980 \quad 5144 \quad 2486$

2623

2632

2639
2647

2647
2659

$1703 \quad 833 \quad 870$

$1710 \quad 836 \quad 874$

$\begin{array}{lll}1724 & 844 & 880\end{array}$

$\begin{array}{lll}1742 & 853 \quad 888\end{array}$

$2993 \quad 5169 \quad 2500$

2668

$1775 \quad 871$

904

Resident population:

projections (mid-year)

\begin{tabular}{rrrrrrrrrrrrrr} 
& C59J & C59K & C59L & C59M & C59N & C59O & C59P & C59Q & C59R & C59S & C59T & C59U & C59V \\
2011 & 62649 & 30842 & 31807 & 55601 & 27412 & 28189 & 3024 & 5233 & 2537 & 2695 & 1815 & 892 & 923 \\
2016 & 64773 & 31986 & 32787 & 57576 & 28474 & 29102 & 3104 & 5324 & 2589 & 2736 & 1874 & 924 & 950 \\
2021 & 66958 & 33134 & 33824 & 59620 & 29547 & 30073 & 3187 & 5411 & 2635 & 2776 & 1927 & 952 & 975 \\
2026 & 69051 & 34210 & 34841 & 61597 & 30563 & 31035 & 3263 & 5483 & 2672 & 2811 & 1971 & 975 & 996 \\
2031 & 70933 & 35162 & 35772 & 63397 & 31473 & 31924 & 3326 & 5532 & 2696 & 2835 & 2005 & 992 & 1012 \\
\hline
\end{tabular}

1 Figures for Northern Ireland are estimated. The population at the Census of

1926 was 1257 thousand (608 thousand males and 649 thousand fe-

males).

2 These projections are 2008-based. See chapter text for more detail.

(t) National Statics: 01329 444661; General Register Office for Scotland Northern Ireland Statistics and Research Agency 


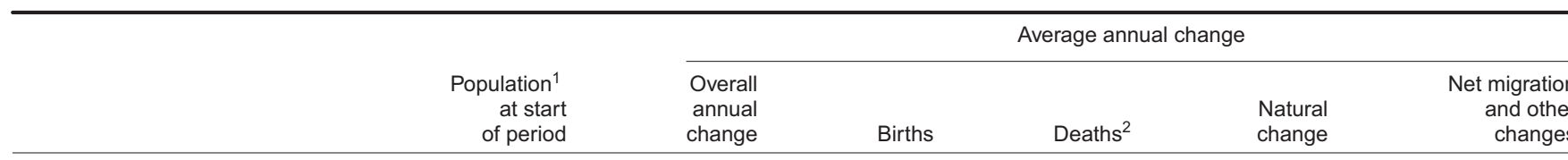

\section{United Kingdom}

$\begin{array}{ll}1901-1911 & 38237 \\ 1911-1921 & 42082 \\ 1921-1931 & 44027 \\ 1931-1951 & 46038 \\ 1951-1961 & 50225 \\ 1961-1971 & \\ 1971-1981 & 52807 \\ 1981-1991 & 55928 \\ 1991-2001 & 56357 \\ 2001-2007 & 57439 \\ 2001-2008 & 59113 \\ 2011-2021 & 59113 \\ \end{array}$

$\begin{array}{rr}385 & 1091 \\ 195 & 975 \\ 201 & 824 \\ 213 & 793 \\ 258 & 839 \\ & \\ 312 & 962 \\ 42 & 736 \\ 108 & 757 \\ 161 & 731 \\ 310 & 710 \\ 324 & 722 \\ 431 & 791\end{array}$

091
975
824
793
839
962
736
757
731
710
722
791

$\begin{array}{rrr}624 & 467 & -82 \\ 689 & 286 & -92 \\ 555 & 268 & -67 \\ 603 & 190 & 22 \\ 593 & 246 & 12 \\ & & \\ 638 & 324 & -12 \\ 666 & 69 & -27 \\ 655 & 103 & 5 \\ 631 & 100 & 61 \\ 591 & 119 & 191 \\ 588 & 134 & 191 \\ & & \\ 544 & 248 & 183\end{array}$

England and Wales

$1901-1911$

$1911-1921$

$1921-1931$

$1931-1951$

$1951-1961$

32528

36070

37887

39952

43758

354
182
207

$1961-1971$

$1971-1981$

$1981-1991$

$1991-2001$

$2001-2007$

$2001-2008$

46196

49152

49634

50748

52360

52360

55601

$2011-2021$

$\begin{array}{rrr}525 & 404 & -50 \\ 584 & 244 & -62 \\ 469 & 224 & -17 \\ 518 & 155 & 38 \\ 516 & 197 & 47 \\ & & \\ 560 & 272 & 23 \\ 585 & 53 & -5 \\ 576 & 89 & 23 \\ 556 & 92 & 63 \\ 520 & 114 & 171 \\ 517 & 127 & 170 \\ & & \end{array}$

Scotland

1901 - 1911

1911 - 1921

$1921-1931$

4472

4761

$1931-1951$

4882

$1951-1961$

4843

$1961-1971$

5096

832

638

664

647

644

477

232

170

1971 - 1981

1981 - 1991

$1991-2001$

2001 - 2007

$2001-2008$

5184

5236

5180

5083

5064

5064

5233

$2011-2021$

Northern Ireland

\begin{tabular}{ll}
$1901-1911$ & 1237 \\
$1911-1921$ & 1251 \\
$1921-1931$ & 1258 \\
$1931-1951$ & 1243 \\
$1951-1961$ & 1371 \\
$1961-1971$ & 1427 \\
$1971-1981$ & 1540 \\
$1981-1991$ & 1543 \\
$1991-2001$ & 1607 \\
$2001-2008$ & 1689 \\
$2011-2021$ & 1815 \\
\hline
\end{tabular}

1237

1251

243

371

1427

543

1815
1

$\begin{array}{rrrr}31 & 23 & 8 & -6 \\ 29 & 22 & 7 & -6 \\ 30 & 21 & 9 & -11 \\ 28 & 18 & 10 & -4 \\ 30 & 15 & 15 & -9\end{array}$

33

28
27

24

23

25
$-82$

22

12

-12
-27
5 (91 91 .

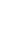

170

1 Census enumerated population up to 1951; mid-year estimates of resident population from 1961 to 2008 and mid-2008-based projections of resident population thereafter.

2 Including deaths of non-civilians and merchant seamen who died outside the country. These numbered 577000 in 1911-1921 and 240000 in 1931-1951 for England and Wales; 74000 in 1911-1921 and 34000 in 1931-1951 for Scotland; and 10000 in 1911-1926 for Northern Ireland. 


\begin{tabular}{|c|c|c|c|c|c|c|c|c|c|c|c|c|c|c|c|c|c|}
\hline & & \multicolumn{16}{|c|}{ United Kingdom } \\
\hline & & \multicolumn{3}{|c|}{$\begin{array}{c}\text { Population } \\
\text { enumerated in Census }\end{array}$} & \multicolumn{9}{|c|}{$\begin{array}{l}\text { Estimated mid-year } \\
\text { resident population }\end{array}$} & \multicolumn{4}{|c|}{$\begin{array}{l}\text { Projected mid-year } \\
\text { resident population }{ }^{1}\end{array}$} \\
\hline Persons: All ages & KGUA & 38237 & 46038 & 50225 & 56357 & 57439 & 59114 & 59557 & 59846 & 60238 & 60587 & 60975 & 61383 & 62649 & 64773 & 66958 & 69051 \\
\hline $\begin{array}{l}\text { Under } 1 \\
1-4 \\
5-9 \\
10-14 \\
15-19\end{array}$ & $\begin{array}{l}\text { KGUK } \\
\text { KABA } \\
\text { KGUN } \\
\text { KGUO } \\
\text { KGUP }\end{array}$ & $\begin{array}{r}938 \\
3443 \\
4106 \\
3934 \\
3826\end{array}$ & $\begin{array}{r}712 \\
2818 \\
3897 \\
3746 \\
3989\end{array}$ & 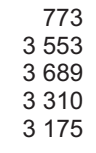 & $\begin{array}{l}730 \\
2726 \\
3677 \\
4470 \\
4735\end{array}$ & $\begin{array}{r}790 \\
3077 \\
3657 \\
3485 \\
3719\end{array}$ & $\begin{array}{r}663 \\
2819 \\
3735 \\
3890 \\
3678\end{array}$ & $\begin{array}{r}680 \\
2706 \\
3650 \\
3896 \\
3856\end{array}$ & $\begin{array}{r}705 \\
2686 \\
3608 \\
3867 \\
3921\end{array}$ & $\begin{array}{l}716 \\
2713 \\
3554 \\
3819 \\
3957\end{array}$ & $\begin{array}{r}732 \\
2765 \\
3490 \\
3751 \\
3996\end{array}$ & $\begin{array}{r}756 \\
2837 \\
3424 \\
3704 \\
4016\end{array}$ & $\begin{array}{r}788 \\
2912 \\
3395 \\
3659 \\
3988\end{array}$ & $\begin{array}{r}775 \\
3108 \\
3508 \\
3500 \\
3832\end{array}$ & 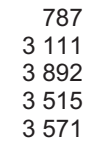 & $\begin{array}{l}801 \\
33190 \\
3906 \\
3898 \\
3585\end{array}$ & $\begin{array}{r}795 \\
3199 \\
3999 \\
3912 \\
3968\end{array}$ \\
\hline $\begin{array}{l}20-29 \\
30-44 \\
45-59 \\
60-64 \\
65-74\end{array}$ & $\begin{array}{l}\text { KABB } \\
\text { KABC } \\
\text { KABD } \\
\text { KGUY } \\
\text { KBCP }\end{array}$ & $\begin{array}{l}6982 \\
7493 \\
4639 \\
1067 \\
1278\end{array}$ & $\begin{array}{l}7865 \\
9717 \\
7979 \\
1897 \\
2461\end{array}$ & $\begin{array}{r}7154 \\
11125 \\
9558 \\
2422 \\
3689\end{array}$ & $\begin{array}{r}8113 \\
10956 \\
9540 \\
2935 \\
5195\end{array}$ & $\begin{array}{r}9138 \\
12125 \\
9500 \\
2888 \\
5067\end{array}$ & $\begin{array}{r}7499 \\
13405 \\
11168 \\
2884 \\
4947\end{array}$ & $\begin{array}{r}7400 \\
13506 \\
11412 \\
2949 \\
5001\end{array}$ & $\begin{array}{r}7496 \\
13460 \\
11507 \\
3027 \\
5028\end{array}$ & $\begin{array}{r}7691 \\
13419 \\
11616 \\
3114 \\
5046\end{array}$ & $\begin{array}{r}7880 \\
13302 \\
11744 \\
3240 \\
5029\end{array}$ & $\begin{array}{r}8107 \\
13141 \\
11728 \\
3483 \\
5058\end{array}$ & $\begin{array}{r}8302 \\
12978 \\
11792 \\
3639 \\
5155\end{array}$ & $\begin{array}{r}8758 \\
12646 \\
12288 \\
3746 \\
5501\end{array}$ & $\begin{array}{r}8920 \\
12600 \\
13080 \\
3442 \\
6344\end{array}$ & $\begin{array}{r}8471 \\
13408 \\
12951 \\
3840 \\
6551\end{array}$ & $\begin{array}{r}8223 \\
14085 \\
12323 \\
4300 \\
6703\end{array}$ \\
\hline $\begin{array}{l}\text { School ages (5-15) } \\
\text { Under } 18 \\
\text { Pensionable ages }{ }^{5}\end{array}$ & $\begin{array}{l}\text { KBWU } \\
\text { KGUD } \\
\text { KFIA }\end{array}$ & $\begin{array}{r}. . \\
2387\end{array}$ & $\begin{array}{r}13120 \\
10557 \\
4421\end{array}$ & $\begin{array}{r}7649 \\
13248 \\
6828\end{array}$ & $\begin{array}{r}9086 \\
14472 \\
10035\end{array}$ & $\begin{array}{r}7818 \\
13120 \\
10557\end{array}$ & $\begin{array}{r}8381 \\
13357 \\
10845\end{array}$ & $\begin{array}{r}8334 \\
13259 \\
11012\end{array}$ & $\begin{array}{r}8254 \\
13219 \\
11117\end{array}$ & $\begin{array}{r}8159 \\
13176 \\
11232\end{array}$ & $\begin{array}{r}8041 \\
13120 \\
11344\end{array}$ & $\begin{array}{r}7917 \\
13111 \\
11562\end{array}$ & $\begin{array}{r}7817 \\
13121 \\
11791\end{array}$ & $\begin{array}{r}7739 \\
13120 \\
12178\end{array}$ & $\begin{array}{r}8077 \\
13375 \\
12493\end{array}$ & $\begin{array}{r}8543 \\
13978 \\
12906\end{array}$ & $\begin{array}{r}8692 \\
14269 \\
13457\end{array}$ \\
\hline Males: All ages & KGWA & 18492 & 22060 & 24118 & 27412 & 27909 & 28832 & 29109 & 29278 & 29497 & 29694 & 29916 & 30151 & 30842 & 31986 & 33134 & 34210 \\
\hline $\begin{array}{l}\text { Under } 1 \\
1-4 \\
5-9 \\
10-14 \\
15-19\end{array}$ & $\begin{array}{l}\text { KGWK } \\
\text { KBCV } \\
\text { KGWN } \\
\text { KGWO } \\
\text { KGWP }\end{array}$ & $\begin{array}{l}471 \\
1719 \\
2052 \\
1972 \\
1898\end{array}$ & $\begin{array}{r}361 \\
1423 \\
1967 \\
1892 \\
1987\end{array}$ & $\begin{array}{r}397 \\
1818 \\
1885 \\
1681 \\
1564\end{array}$ & 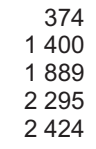 & $\begin{array}{r}403 \\
1572 \\
1871 \\
1784 \\
1905\end{array}$ & $\begin{array}{r}338 \\
1445 \\
1913 \\
1993 \\
1879\end{array}$ & $\begin{array}{r}349 \\
1384 \\
1870 \\
1998 \\
1989\end{array}$ & $\begin{array}{r}362 \\
1376 \\
1847 \\
1985 \\
2018\end{array}$ & $\begin{array}{r}367 \\
1389 \\
1819 \\
1962 \\
2030\end{array}$ & $\begin{array}{r}374 \\
1416 \\
1785 \\
1924 \\
2060\end{array}$ & $\begin{array}{r}387 \\
1453 \\
1750 \\
1898 \\
2069\end{array}$ & $\begin{array}{r}404 \\
1492 \\
1737 \\
1873 \\
2049\end{array}$ & $\begin{array}{r}397 \\
1591 \\
1795 \\
1791 \\
1966\end{array}$ & $\begin{array}{r}403 \\
1592 \\
1989 \\
1802 \\
1831\end{array}$ & $\begin{array}{r}410 \\
1632 \\
1995 \\
1995 \\
1841\end{array}$ & $\begin{array}{r}407 \\
1637 \\
2043 \\
2002 \\
2034\end{array}$ \\
\hline $\begin{array}{l}20-29 \\
30-44 \\
45-59 \\
60-64 \\
65-74\end{array}$ & $\begin{array}{l}\text { KBCW } \\
\text { KBCX } \\
\text { KBUU } \\
\text { KGWY } \\
\text { KBWL }\end{array}$ & $\begin{array}{r}3293 \\
3597 \\
2215 \\
490 \\
565\end{array}$ & $\begin{array}{r}3818 \\
4495 \\
3753 \\
894 \\
1099\end{array}$ & $\begin{array}{l}3509 \\
5461 \\
4493 \\
1061 \\
1560\end{array}$ & $\begin{array}{l}4103 \\
5513 \\
4711 \\
1376 \\
2264\end{array}$ & $\begin{array}{l}4578 \\
6045 \\
4732 \\
1390 \\
2272\end{array}$ & $\begin{array}{l}3744 \\
6645 \\
5534 \\
1412 \\
2308\end{array}$ & $\begin{array}{l}3709 \\
6695 \\
5646 \\
1440 \\
2347\end{array}$ & $\begin{array}{l}3773 \\
6669 \\
5691 \\
1479 \\
2365\end{array}$ & $\begin{array}{l}3878 \\
6655 \\
5745 \\
1522 \\
2380\end{array}$ & $\begin{array}{l}3978 \\
6597 \\
5804 \\
1584 \\
2379\end{array}$ & $\begin{array}{l}4116 \\
6522 \\
5786 \\
1701 \\
2398\end{array}$ & $\begin{array}{l}4235 \\
6449 \\
5815 \\
1778 \\
2447\end{array}$ & $\begin{array}{l}4479 \\
6311 \\
6048 \\
1824 \\
2623\end{array}$ & $\begin{array}{l}4555 \\
6353 \\
6431 \\
1673 \\
3032\end{array}$ & $\begin{array}{l}4318 \\
6812 \\
6373 \\
11864 \\
3127\end{array}$ & $\begin{array}{l}4193 \\
7180 \\
6099 \\
2078 \\
32201\end{array}$ \\
\hline $\begin{array}{l}\text { School ages (5-15) } \\
\text { Under } 18 \\
\text { Pensionable ages }{ }^{5}\end{array}$ & $\begin{array}{l}\text { KBWV } \\
\text { KGWD } \\
\text { KFIB }\end{array}$ & $\begin{array}{r}. . \\
. . \\
785\end{array}$ & $\begin{array}{l}3630 \\
1471\end{array}$ & $\begin{array}{l}3895 \\
6753 \\
2247\end{array}$ & $\begin{array}{l}4666 \\
7430 \\
3327\end{array}$ & $\begin{array}{l}4001 \\
6711 \\
3630\end{array}$ & $\begin{array}{l}4294 \\
6845 \\
3928\end{array}$ & $\begin{array}{l}4273 \\
6799 \\
4028\end{array}$ & $\begin{array}{l}4233 \\
6780 \\
4078\end{array}$ & $\begin{array}{l}4185 \\
6756 \\
4130\end{array}$ & $\begin{array}{l}4122 \\
6727 \\
4171\end{array}$ & $\begin{array}{l}4054 \\
6721 \\
4233\end{array}$ & $\begin{array}{l}4002 \\
6723 \\
4321\end{array}$ & $\begin{array}{l}3961 \\
6719 \\
4639\end{array}$ & $\begin{array}{l}4134 \\
6848 \\
5358\end{array}$ & $\begin{array}{l}4369 \\
7154 \\
5893\end{array}$ & $\begin{array}{l}4445 \\
7301 \\
6158\end{array}$ \\
\hline Females: All ages & KGYA & 19745 & 23978 & 26107 & 28946 & 29530 & 30281 & 30449 & 30568 & 30741 & 30893 & 31059 & 31232 & 31807 & 32787 & 33824 & 34841 \\
\hline $\begin{array}{l}\text { Under } 1 \\
1-4 \\
5-9 \\
10-14 \\
15-19\end{array}$ & $\begin{array}{l}\text { KGYK } \\
\text { KBWN } \\
\text { KGYN } \\
\text { KGYO } \\
\text { KGYP }\end{array}$ & $\begin{array}{l}466 \\
1724 \\
2054 \\
1962 \\
1928\end{array}$ & $\begin{array}{l}1397 \\
1930 \\
1854 \\
2002\end{array}$ & $\begin{array}{r}376 \\
1735 \\
1804 \\
1629 \\
1611\end{array}$ & $\begin{array}{l}356 \\
1327 \\
1788 \\
2175 \\
2311\end{array}$ & $\begin{array}{r}387 \\
1505 \\
1786 \\
1701 \\
1815\end{array}$ & $\begin{array}{r}324 \\
1375 \\
1822 \\
1897 \\
1799\end{array}$ & $\begin{array}{r}331 \\
1322 \\
1781 \\
1897 \\
1867\end{array}$ & $\begin{array}{r}343 \\
1310 \\
1761 \\
1882 \\
1903\end{array}$ & $\begin{array}{r}349 \\
1324 \\
1735 \\
1857 \\
1927\end{array}$ & $\begin{array}{l}1349 \\
1705 \\
1827 \\
1936\end{array}$ & $\begin{array}{r}368 \\
1383 \\
1674 \\
1806 \\
1947\end{array}$ & $\begin{array}{r}385 \\
1420 \\
1658 \\
1785 \\
1939\end{array}$ & $\begin{array}{r}378 \\
1517 \\
1713 \\
1709 \\
1866\end{array}$ & $\begin{array}{r}384 \\
1519 \\
1903 \\
1714 \\
1740\end{array}$ & $\begin{array}{l}391 \\
1558 \\
1911 \\
1903 \\
1745\end{array}$ & $\begin{array}{r}388 \\
1562 \\
1956 \\
1911 \\
1934\end{array}$ \\
\hline $\begin{array}{l}20-29 \\
30-44 \\
45-59 \\
60-64 \\
65-74\end{array}$ & $\begin{array}{l}\text { KBWO } \\
\text { KBWP } \\
\text { KBWR } \\
\text { KGYY } \\
\text { KBWS }\end{array}$ & $\begin{array}{r}3690 \\
3895 \\
2424 \\
577 \\
713\end{array}$ & $\begin{array}{l}5222 \\
4226 \\
1003 \\
1361\end{array}$ & $\begin{array}{ll}3 & 644 \\
5 & 663 \\
5 & 065 \\
1 & 361 \\
2 & 127\end{array}$ & $\begin{array}{l}4009 \\
5442 \\
4829 \\
1559 \\
2931\end{array}$ & $\begin{array}{l}4560 \\
6080 \\
4769 \\
1498 \\
2795\end{array}$ & $\begin{array}{l}3755 \\
6760 \\
5634 \\
1473 \\
2640\end{array}$ & $\begin{array}{l}3691 \\
6811 \\
5766 \\
1509 \\
2654\end{array}$ & $\begin{array}{l}3723 \\
6792 \\
5816 \\
1548 \\
2662\end{array}$ & $\begin{array}{ll}3 & 813 \\
6 & 764 \\
5 & 871 \\
1 & 591 \\
2 & 666\end{array}$ & $\begin{array}{l}3902 \\
6706 \\
5940 \\
1656 \\
2650\end{array}$ & $\begin{array}{l}3990 \\
6620 \\
5942 \\
1782 \\
2660\end{array}$ & $\begin{array}{l}4067 \\
6529 \\
5978 \\
1861 \\
2708\end{array}$ & $\begin{array}{l}4278 \\
6335 \\
6240 \\
1923 \\
2878\end{array}$ & $\begin{array}{l}4366 \\
6247 \\
6649 \\
1769 \\
3312\end{array}$ & $\begin{array}{l}4153 \\
6596 \\
6579 \\
1976 \\
3424\end{array}$ & 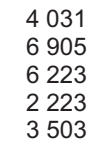 \\
\hline $\begin{array}{l}75-84 \\
85 \text { and over }\end{array}$ & $\begin{array}{l}\text { KBWT } \\
\text { KGZD }\end{array}$ & $\begin{array}{r}274 \\
38\end{array}$ & 77 & $\begin{array}{l}937 \\
154\end{array}$ & $\begin{array}{r}1756 \\
462\end{array}$ & $\begin{array}{r}1972 \\
661\end{array}$ & $\begin{array}{r}1987 \\
817\end{array}$ & $\begin{array}{r}2029 \\
792\end{array}$ & $\begin{array}{r}2040 \\
789\end{array}$ & $\begin{array}{r}020 \\
825\end{array}$ & $\begin{array}{r}2002 \\
864\end{array}$ & $\begin{array}{r}1992 \\
895\end{array}$ & $\begin{array}{r}1988 \\
914\end{array}$ & $\begin{array}{r}2005 \\
965\end{array}$ & $\begin{array}{l}2113 \\
1072\end{array}$ & $\begin{array}{l}2371 \\
1218\end{array}$ & $\begin{array}{l}2775 \\
1431\end{array}$ \\
\hline $\begin{array}{l}\text { School ages }(5-15) \\
\text { Under } 18 \\
\text { Pensionable ages }{ }^{5}\end{array}$ & $\begin{array}{l}\text { KBWW } \\
\text { KGYD } \\
\text { KFIC }\end{array}$ & $\begin{array}{r}. . \\
1601\end{array}$ & $\begin{array}{l}6409 \\
6927 \\
2950\end{array}$ & $\begin{array}{l}3753 \\
6495 \\
4580\end{array}$ & $\begin{array}{l}4421 \\
7042 \\
6708\end{array}$ & $\begin{array}{l}3817 \\
6409 \\
6927\end{array}$ & $\begin{array}{l}4087 \\
6512 \\
6917\end{array}$ & $\begin{array}{l}4061 \\
6460 \\
6984\end{array}$ & $\begin{array}{l}4022 \\
6439 \\
7039\end{array}$ & $\begin{array}{l}3974 \\
6419 \\
7102\end{array}$ & $\begin{array}{l}3919 \\
6393 \\
7172\end{array}$ & $\begin{array}{l}3863 \\
6390 \\
7329\end{array}$ & $\begin{array}{l}3815 \\
6398 \\
7471\end{array}$ & $\begin{array}{l}3778 \\
6402 \\
7539\end{array}$ & $\begin{array}{l}3943 \\
6527 \\
7135\end{array}$ & $\begin{array}{l}4174 \\
6824 \\
7013\end{array}$ & $\begin{array}{l}4247 \\
6969 \\
72299\end{array}$ \\
\hline
\end{tabular}




\section{3 Age distribution of the resident population: by sex and country

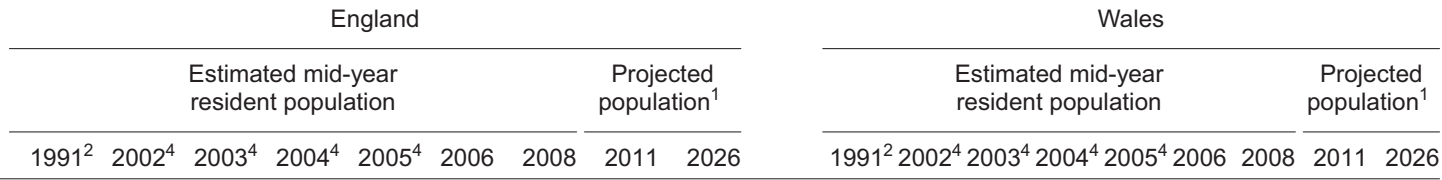

Persons: All ages KCCI 478754965249866501115046650763514465257758334 KERY 287329202931294629542966299330243263

\begin{tabular}{|c|c|c|c|c|c|c|c|c|c|c|c|c|c|c|c|c|c|c|c|}
\hline Under 1 & $\mathrm{KCCJ}$ & 660 & 559 & 576 & 597 & 606 & 620 & 668 & 657 & $682 \mathrm{KFAC}$ & 38 & 30 & 31 & 32 & 32 & 33 & 35 & 35 & 35 \\
\hline $1-4$ & $\mathrm{KCCK}$ & 2560 & 2313 & 2275 & 2262 & 2289 & 2335 & 2462 & 2632 & 2735 кFBX & 153 & 132 & 129 & 127 & 126 & 127 & 133 & 140 & 143 \\
\hline $5-9$ & $\mathrm{KCCL}$ & 3019 & 3084 & 3055 & 3020 & 2976 & 2922 & 2849 & 2959 & $3402 \mathrm{KFCA}$ & 186 & 183 & 180 & 178 & 175 & 172 & 163 & 163 & 183 \\
\hline $10-$ & $\mathrm{KCCM}$ & 2865 & 3264 & 3250 & 3225 & 3185 & 3130 & 3055 & 2926 & $3313 \mathrm{KFCB}$ & 177 & 197 & 197 & 195 & 193 & 189 & 3 & 174 & 82 \\
\hline $15-1$ & $\mathrm{KCCN}$ & 3067 & 3115 & 3203 & 3261 & 3297 & 3334 & 3331 & 3199 & $3354 \mathrm{KFCC}$ & 187 & 190 & 196 & 199 & 200 & 202 & 203 & 194 & 186 \\
\hline & CEG & 1 & 244 & 6232 & 315 & 483 & 6633 & 974 & 7385 & 7009 KFCD & 415 & 333 & 334 & 340 & 348 & 359 & 4 & 401 & \\
\hline & $\mathrm{KCEH}$ & 10147 & 11347 & 11369 & 11337 & 11318 & 11230 & 10975 & 10711 & $3 \mathrm{KFCE}$ & 583 & 610 & 608 & 606 & 599 & 590 & I & 544 & \\
\hline 45 & $\mathrm{KCEQ}$ & 7920 & 9439 & 9522 & 9591 & 9675 & 9777 & 9809 & 10235 & $2 \mathrm{KFCF}$ & 486 & 578 & 582 & 586 & 589 & 592 & 9 & 602 & \\
\hline $60-$ & KCEW & 2399 & 2399 & 2445 & 2509 & 2586 & 2697 & 3039 & 3118 & 3583 KFCG & 154 & 156 & 161 & 166 & 171 & 177 & 197 & 203 & \\
\hline $5-74$ & KCGD & 4222 & 4129 & 4155 & 4175 & 4189 & 4171 & 4274 & 4572 & $11 \mathrm{KFCH}$ & 284 & 265 & 268 & 270 & 271 & 273 & 282 & 303 & \\
\hline 政 & KCJG & 2626 & 2000 & 2850 & 20. & 2000 & 2860 & & $2 s$ & & דות & 185 & 187 & 188 & 186 & 186 & 185 & 100 & \\
\hline 85 and ov & KCKJ & 739 & 95 & 936 & 942 & 996 & 1055 & 1135 & 1226 & $2032 \mathrm{KFCK}$ & 45 & 59 & 59 & 60 & 63 & 67 & 72 & 79 & \\
\hline $\mathrm{ch}$ & KCWX & & 34 & 960 & 95 & 17 & 19 & & & FCL & & 419 & 417 & 413 & 407 & 401 & & 37 & \\
\hline Under 18 & KCWY & 10840 & 11119 & 11089 & 11064 & 11036 & 10997 & 11008 & 1103 & $12130 \mathrm{KFCM}$ & 662 & 659 & 654 & 651 & 646 & 641 & 634 & 623 & \\
\hline Pensionable ages ${ }^{5}$ & KEAA & 8827 & 911 & 9188 & 9273 & 9370 & 9462 & 9839 & 10164 & 11207 KFEB & 573 & 589 & 595 & 602 & 608 & 615 & 639 & 661 & \\
\hline
\end{tabular}

Males: All ages $\quad$ KEAB 232912429024419245632475824926253192593228952 KFEI 139114141423143214391445146114801610

\begin{tabular}{|c|c|c|c|c|c|c|c|c|c|c|c|c|c|c|c|c|c|c|c|}
\hline Under 1 & KEAC & 336 & 286 & 296 & 306 & 310 & 317 & 342 & 337 & $349 \mathrm{KFEJ}$ & 20 & 16 & 16 & 16 & 17 & 17 & 18 & 18 & \\
\hline $1-4$ & KEAD & 307 & 1182 & 1163 & 1159 & 1172 & 1196 & 1260 & 1347 & $1400 \mathrm{KFEK}$ & 78 & 68 & 66 & 65 & 65 & 65 & 68 & 72 & \\
\hline $5-9$ & KEAE & 545 & 1581 & 1564 & 1546 & 1522 & 1493 & 1457 & 1513 & $1738 \mathrm{KFEL}$ & 95 & 94 & 92 & 91 & 90 & 88 & 84 & 84 & \\
\hline $10-14$ & KEAF & 467 & 1672 & 1667 & 1657 & 1638 & 1606 & 1564 & 1497 & $1695 \mathrm{KFFA}$ & 91 & 101 & 101 & 100 & 99 & 97 & 94 & 89 & \\
\hline $15-19$ & KECA & 572 & 1607 & 1654 & 1679 & 1691 & 1720 & 1712 & 1642 & $1720 \mathrm{KFFN}$ & 95 & 97 & 100 & 102 & 103 & 104 & 104 & 99 & \\
\hline - & $E C B$ & 835 & 130 & 26 & 3182 & 3270 & 349 & 3561 & 3780 & 3569 KFHA & 07 & 164 & 166 & 70 & 6 & 81 & 195 & & \\
\hline $30-$ & $\mathrm{KECC}$ & 064 & 5644 & 658 & 5639 & 5637 & 5591 & 5475 & 5363 & $6126 \mathrm{KFHB}$ & 289 & 297 & 296 & 295 & 92 & 287 & 276 & 66 & \\
\hline $5-$ & KECD & 3957 & 4673 & 715 & 4748 & 4791 & 4839 & 4845 & 5051 & $5166 \mathrm{KFHW}$ & 242 & 285 & 287 & 288 & 290 & 291 & 288 & 993 & \\
\hline 4 & ECE & 159 & 1176 & 1197 & 1228 & 1267 & 1320 & 1485 & 1518 & $1738 \mathrm{KFQO}$ & 74 & 77 & 79 & 82 & 84 & 87 & 97 & 00 & \\
\hline $65-74$ & $\mathrm{KECF}$ & 1900 & 1942 & 1958 & 1972 & 1984 & 1981 & 2035 & 2183 & $2650 \mathrm{KFQV}$ & 128 & 125 & 127 & 128 & 129 & 130 & 135 & 146 & \\
\hline $5-$ & $\mathrm{ECG}$ & 970 & 1128 & 115 & 1172 & 1179 & 1190 & 1222 & 1290 & 1970 & 60 & 74 & 75 & 76 & 77 & 77 & 78 & 82 & \\
\hline 85 and ov & $\mathrm{KECH}$ & 181 & 269 & 67 & 274 & 298 & 324 & 361 & 411 & 832 KFUL & 11 & 16 & 16 & 17 & 19 & 20 & 23 & 6 & \\
\hline ch & ECI & 295 & 35 & & 35 & 34 & & & 33 & JV & & 21 & 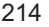 & 21 & & 6 & 98 & 2 & \\
\hline Under $1 \varepsilon$ & KECJ & 5545 & 5699 & 5686 & 5675 & 5658 & 5638 & 5640 & 5650 & $6207 \mathrm{KFVE}$ & 33 & 338 & 336 & 335 & 332 & 329 & 326 & 0 & \\
\hline Pensionable ages ${ }^{5}$ & $\mathrm{KECK}$ & 3050 & 3339 & 3379 & 3419 & 3461 & 3494 & 3617 & 3885 & $5137 \mathrm{KFVF}$ & 198 & 215 & 218 & 221 & 224 & 227 & 236 & 254 & \\
\hline
\end{tabular}

Females: All ages KEJV 245842536225448255482570825837261272664529382 KFVL 148215061508151415151521153215451653

\begin{tabular}{|c|c|c|c|c|c|c|c|c|c|c|c|c|c|c|c|c|c|c|c|}
\hline Under 1 & KEJW & 324 & 273 & 280 & 291 & 296 & 303 & 326 & 321 & 333 KFYW & 19 & 15 & 15 & 15 & 16 & 16 & 17 & 17 & \\
\hline $1-4$ & KEJX & 1253 & 1131 & 1112 & 1103 & 1117 & 1139 & 1201 & 1285 & $1336 \mathrm{kFZJ}$ & 75 & 65 & 63 & 62 & 61 & 62 & 65 & 68 & \\
\hline 0 & KEKP & 474 & 1503 & 490 & 1474 & 1454 & 428 & 1392 & 1445 & 664 KGCK & 91 & 8 & 8 & 87 & 85 & 84 & 9 & 9 & \\
\hline $0-14$ & KEKQ & 399 & 1591 & 583 & 1569 & 1547 & 523 & 1491 & 1429 & $617 \mathrm{KGCM}$ & 8 & & & & 9 & 2 & 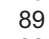 & 4 & \\
\hline $5-$ & KEKR & 495 & 508 & 5 & 1582 & 606 & 1615 & 1619 & 1557 & $4 \mathrm{KGCN}$ & & & & & 9 & 0 & 9 & 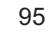 & \\
\hline - & EKS & 816 & 11 & 106 & 3133 & 213 & 328 & 3414 & 3606 & $440 \mathrm{KGCO}$ & 208 & 68 & 67 & 70 & 72 & 78 & 89 & 6 & \\
\hline-4 & KENR & 083 & 5703 & 5711 & 5699 & 5682 & 5638 & 5499 & 5348 & 5877 KGCP & 294 & 313 & 312 & 311 & 307 & 3 & 91 & 8 & \\
\hline - & KEOQ & 3964 & 4767 & 4808 & 4843 & 4885 & 4938 & 4963 & 5184 & 5226 KGGZ & 244 & 293 & 295 & 298 & 299 & 301 & 01 & 08 & \\
\hline - & KEOZ & 1239 & 1223 & 1248 & 1280 & 1319 & 1377 & 1554 & 1600 & 1846 KGIY & 80 & 80 & 82 & 84 & 87 & 90 & 00 & 04 & \\
\hline $5-74$ & KEQJ & 2323 & 2187 & 2197 & 2203 & 2206 & 2190 & 2239 & 2388 & 2891 KGKR & 156 & 140 & 141 & 142 & 142 & 143 & 147 & 157 & \\
\hline $15-$ & KEQK & 1656 & 1676 & 1696 & 1703 & 1686 & 167 & 1655 & 1668 & $T Q$ & 104 & 111 & 112 & 112 & 110 & 108 & 107 & 106 & \\
\hline 85 and ov & KEQL & 558 & 687 & 669 & 667 & 697 & 731 & 774 & 815 & $1200 \mathrm{kGTZ}$ & 34 & 43 & 42 & 42 & 44 & 47 & 50 & 52 & \\
\hline CII & $\mathrm{KEQM}$ & 1 & $34 c$ & & 3359 & . & & & & VG & 19 & 20 & 203 & 201 & & & 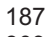 & 18 & \\
\hline Unde & KEQN & 5295 & 5419 & 5403 & 5389 & 5377 & 5358 & 5368 & 5385 & $5924 \mathrm{KGVH}$ & 323 & 321 & 318 & 317 & 31 & 311 & 309 & 303 & \\
\hline Pensionable ages & KEQO & 5777 & 772 & 5809 & 5854 & 5908 & 5968 & 6222 & 6279 & 6070 KGVK & 375 & 374 & 377 & 380 & 383 & 387 & 403 & 407 & \\
\hline
\end{tabular}




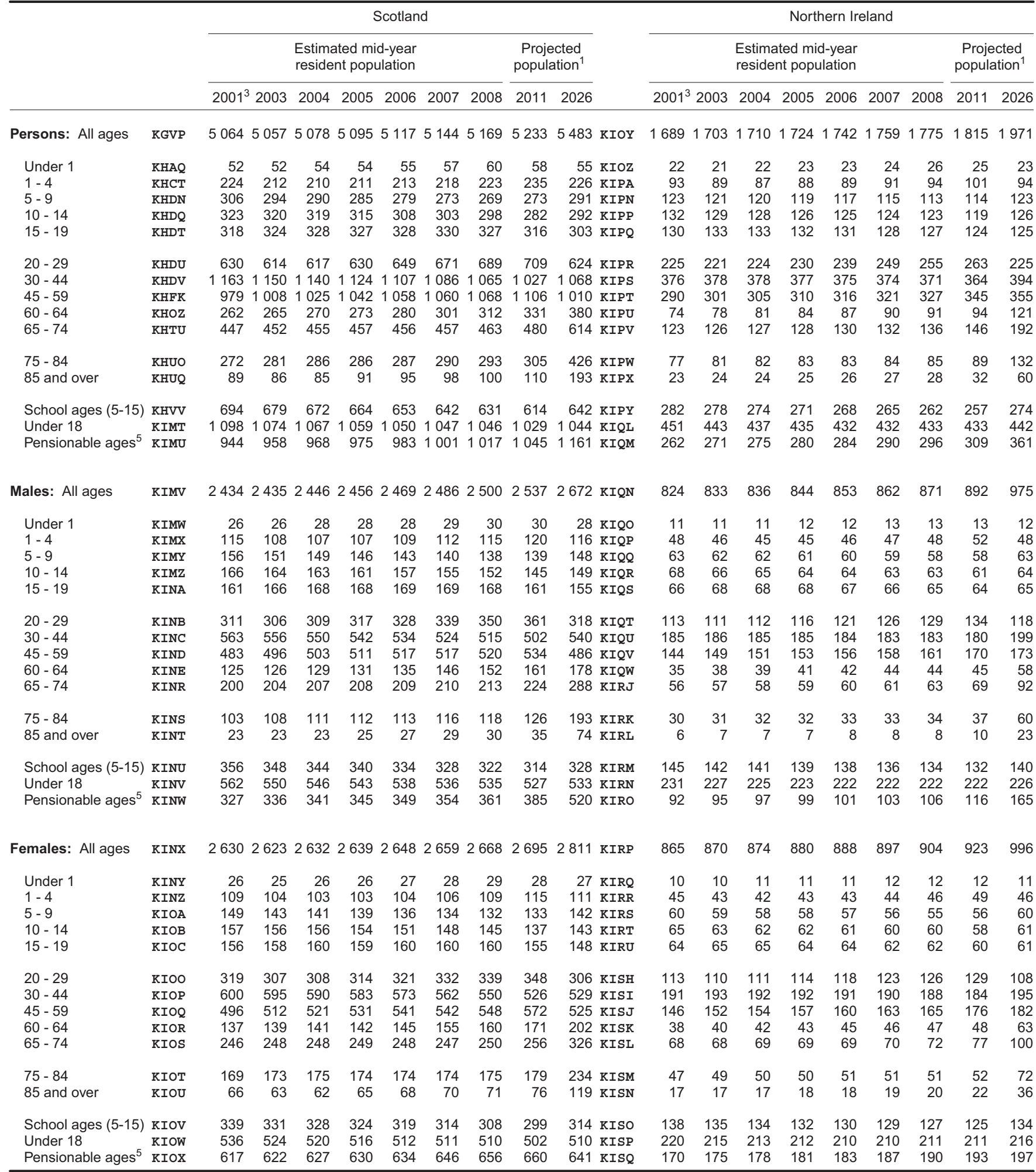

1 2008-based national population projections. See explanatory notes at beginning of chapter for further details.

2 Data for mid 1991 for UK, England and Wales and Scotland are revised in Sources: Office for National Statistics: 01329444661 General Register Office for Scotland; light of the results of the 2001 Census.

3 Data for mid-2001 were revised as a result of local authority population studies.

4 England \& Wales population estimates for mid-2003 to mid-2005 were revised in August 2007 to take account of improved estimates of international migration.

5 The pensionable age population is that over state retirement age. The 2011 figures take account of planned changes in retirement age from 65 for men and 60 for women at present to 65 for both sexes. This change will be phased in between April 2010 and March 2020. 


\begin{tabular}{|c|c|c|c|c|c|c|c|c|c|c|c|c|c|c|c|c|c|c|c|}
\hline & & & \multicolumn{9}{|c|}{ Males } & \multicolumn{8}{|c|}{ Females } \\
\hline & & & 2001 & $2002^{1}$ & $2003^{1}$ & $2004^{1}$ & $2005^{1}$ & $2006^{1}$ & $2007^{1}$ & $2008^{1}$ & & 2001 & $2002^{1}$ & $2003^{1}$ & $2004^{1}$ & $2005^{1}$ & $2006^{1}$ & $2007^{1}$ & 12008 \\
\hline \multicolumn{20}{|c|}{ All ages: } \\
\hline & Single & KRPL & 12270 & 12408 & 12550 & 12714 & 12893 & 13078 & 13279 & 13472 & KUBS & 10917 & 11035 & 11167 & 11310 & 11494 & 11673 & 11853 & 12045 \\
\hline & Married & KRPM & 1090 & 11043 & 10995 & 10941 & 10923 & 10881 & 10851 & 10849 & $\mathrm{KVCC}$ & 11150 & 11094 & 11033 & 10980 & 10943 & 10893 & 10851 & 10812 \\
\hline & Widowed & KRPN & 733 & 730 & 726 & 722 & 719 & 716 & 715 & 713 & KVCD & 2745 & 2709 & 2669 & 2628 & 2588 & 2548 & 2511 & 2477 \\
\hline & Divorced & KRPO & 1482 & 1524 & 1571 & 1617 & 1662 & 1696 & 1724 & 1746 & $\mathrm{KVCE}$ & 1975 & 2031 & 2087 & 2144 & 2198 & 2244 & 2289 & 2326 \\
\hline \multicolumn{20}{|c|}{ Age groups: } \\
\hline $0-14:$ & Single & KRPP & 5036 & 4999 & 4967 & 4941 & 4912 & 4880 & 4872 & 4888 & $\mathrm{KVCF}$ & 4796 & 4763 & 4726 & 4696 & 4670 & 4648 & 4644 & 4660 \\
\hline \multirow[t]{4}{*}{$15-19:$} & Single & $\mathrm{KRPQ}$ & 1645 & 1698 & 1749 & 1778 & 1792 & 1822 & 1832 & 1814 & KVCG & 1560 & 1587 & 1631 & 1667 & 1693 & 1706 & 1718 & 1711 \\
\hline & Married & KRPR & 5 & 5 & 4 & 3 & 2 & 2 & 2 & 2 & $\mathrm{KVCH}$ & 16 & 13 & 12 & 11 & 10 & 7 & 7 & 7 \\
\hline & Widowed & KRPS & 1 & 1 & 1 & - & - & - & - & - & KVCI & 1 & 1 & 1 & - & - & - & - & - \\
\hline & Divorced & KRPT & 1 & 1 & 1 & - & - & - & - & - & KVCJ & 1 & 1 & - & - & - & - & - & - \\
\hline \multirow{3}{*}{$20-24:$} & Single & KRPU & 1501 & 1530 & 1568 & 1632 & 1693 & 1741 & 1813 & 1855 & KVCK & 1390 & 1427 & 1459 & 1491 & 1539 & 1591 & 1637 & 1689 \\
\hline & Married & KRPV & 74 & 73 & 74 & 75 & 73 & 67 & 64 & 61 & KVCL & 178 & 170 & 166 & 163 & 157 & 146 & 136 & 126 \\
\hline & Divorced & KRPX & 3 & 3 & 3 & 3 & 3 & 3 & 3 & 2 & KVCN & 8 & 8 & 8 & 8 & 8 & 7 & 6 & 6 \\
\hline \multirow[t]{4}{*}{$25-34:$} & Single & KRPY & 2227 & 2229 & 2230 & 2245 & 2292 & 2343 & 2383 & 2452 & KVCO & 1770 & 1788 & 1811 & 1846 & 1906 & 1961 & 2000 & 2046 \\
\hline & Married & KRPZ & 1391 & 1311 & 1237 & 1173 & 1125 & 1068 & 1027 & 1006 & KVCP & 1768 & 1671 & 1584 & 1506 & 1452 & 1386 & 1326 & 1281 \\
\hline & Widowed & KRQA & 3 & 3 & 3 & 3 & 3 & 2 & 2 & 3 & KVCQ & 10 & 9 & 8 & 7 & 7 & 7 & 7 & 6 \\
\hline & Divorced & $\mathrm{KRQB}$ & 136 & 129 & 124 & 118 & 111 & 103 & 96 & 90 & KVCR & 231 & 217 & 206 & 193 & 182 & 169 & 158 & 147 \\
\hline \multirow[t]{4}{*}{$35-44:$} & Single & $\mathrm{KRQC}$ & 963 & 1027 & 1080 & 1126 & 1173 & 1219 & 1259 & 1289 & KVEH & 692 & 749 & 801 & 853 & 903 & 955 & 1004 & 1044 \\
\hline & Married & KRQD & 2494 & 2499 & 2488 & 2466 & 2452 & 2419 & 2385 & 2333 & KVEI & 2649 & 2653 & 2638 & 2623 & 2597 & 2564 & 2525 & 2463 \\
\hline & Widowed & $\mathrm{KRQE}$ & 12 & 12 & 12 & 11 & 11 & 11 & 10 & 10 & KVEJ & 36 & 35 & 33 & 32 & 31 & 30 & 29 & 27 \\
\hline & Divorced & $\mathrm{KRQF}$ & 411 & 420 & 427 & 433 & 436 & 431 & 422 & 407 & KVEK & 558 & 570 & 579 & 588 & 590 & 585 & 574 & 555 \\
\hline \multirow[t]{4}{*}{$45-54:$} & Single & KRQG & 419 & 432 & 450 & 473 & 499 & 530 & 563 & 601 & KVEL & 256 & 271 & 288 & 310 & 334 & 363 & 397 & 436 \\
\hline & Married & KUAR & 2511 & 2432 & 2383 & 2356 & 2348 & 2355 & 2365 & 2390 & KVEM & 2548 & 2477 & 2428 & 2401 & 2391 & 2398 & 2413 & 2437 \\
\hline & Widowed & KUBA & 37 & 35 & 34 & 32 & 31 & 30 & 29 & 29 & KVEN & 111 & 105 & 99 & 96 & 92 & 90 & 87 & 85 \\
\hline & Divorced & KUBB & 448 & 451 & 461 & 474 & 489 & 504 & 518 & 532 & KVEO & 557 & 565 & 576 & 591 & 609 & 630 & 654 & 676 \\
\hline \multirow[t]{3}{*}{55 - 59: } & Single & KUBC & 128 & 141 & 150 & 158 & 164 & 170 & 170 & 171 & KVEP & 74 & 81 & 86 & 91 & 96 & 102 & 104 & 108 \\
\hline & Married & KUBD & 1156 & 1238 & 1278 & 1288 & 1285 & 1271 & 1201 & 1148 & KVEQ & 1125 & 1212 & 1256 & 1271 & 1271 & 1260 & 1197 & 1145 \\
\hline & Widowed & KUBE & 34 & 36 & 36 & 37 & 37 & 36 & 34 & 32 & KVER & 112 & 115 & 114 & 111 & 109 & 106 & 98 & 91 \\
\hline \multirow{3}{*}{60 - 64: } & Married & KUBH & 980 & 977 & 991 & 1013 & 1039 & 1077 & 1150 & 1196 & KVEU & 906 & 910 & 932 & 958 & 988 & 1029 & 1110 & 1158 \\
\hline & Widowed & KUBI & 50 & 49 & 48 & 47 & 47 & 48 & 51 & 52 & KVEV & 178 & 172 & 167 & 163 & 160 & 159 & 162 & 162 \\
\hline & Divorced & KUBJ & 125 & 131 & 138 & 147 & 156 & 168 & 186 & 200 & KVEW & 151 & 158 & 169 & 180 & 193 & 210 & 234 & 254 \\
\hline \multirow[t]{4}{*}{$65-74:$} & Single & KUBK & 155 & 154 & 153 & 152 & 151 & 149 & 150 & 152 & KMGN & 130 & 126 & 123 & 120 & 117 & 113 & 111 & 111 \\
\hline & Married & KUBL & 1569 & 1581 & 1595 & 1606 & 1614 & 1610 & 1620 & 1649 & KMGO & 1322 & 1336 & 1356 & 1373 & 1389 & 1393 & 1410 & 1449 \\
\hline & Widowed & KUBM & 188 & 183 & 178 & 174 & 170 & 165 & 162 & 160 & KMGP & 697 & 675 & 655 & 633 & 611 & 584 & 563 & 550 \\
\hline & Divorced & KUBN & 139 & 148 & 158 & 168 & 178 & 186 & 196 & 208 & KMGQ & 177 & 190 & 204 & 218 & 232 & 243 & 257 & 275 \\
\hline \multicolumn{20}{|l|}{75 and } \\
\hline \multirow[t]{4}{*}{ over: } & Single & KUBO & 99 & 101 & 103 & 106 & 109 & 111 & 114 & 116 & KMGR & 188 & 182 & 177 & 173 & 169 & 166 & 163 & 160 \\
\hline & Married & KUBP & 909 & 928 & 944 & 962 & 984 & 1011 & 1038 & 1064 & KMGS & 639 & 651 & 661 & 673 & 689 & 709 & 728 & 746 \\
\hline & Widowed & KUBQ & 407 & 411 & 414 & 417 & 419 & 422 & 425 & 427 & KMGT & 1598 & 1597 & 1590 & 1583 & 1576 & 1571 & 1564 & 1553 \\
\hline & Divorced & KUBR & 44 & 48 & 51 & 56 & 61 & 66 & 71 & 77 & KMGU & 81 & 86 & 90 & 96 & 103 & 110 & 118 & 126 \\
\hline
\end{tabular}




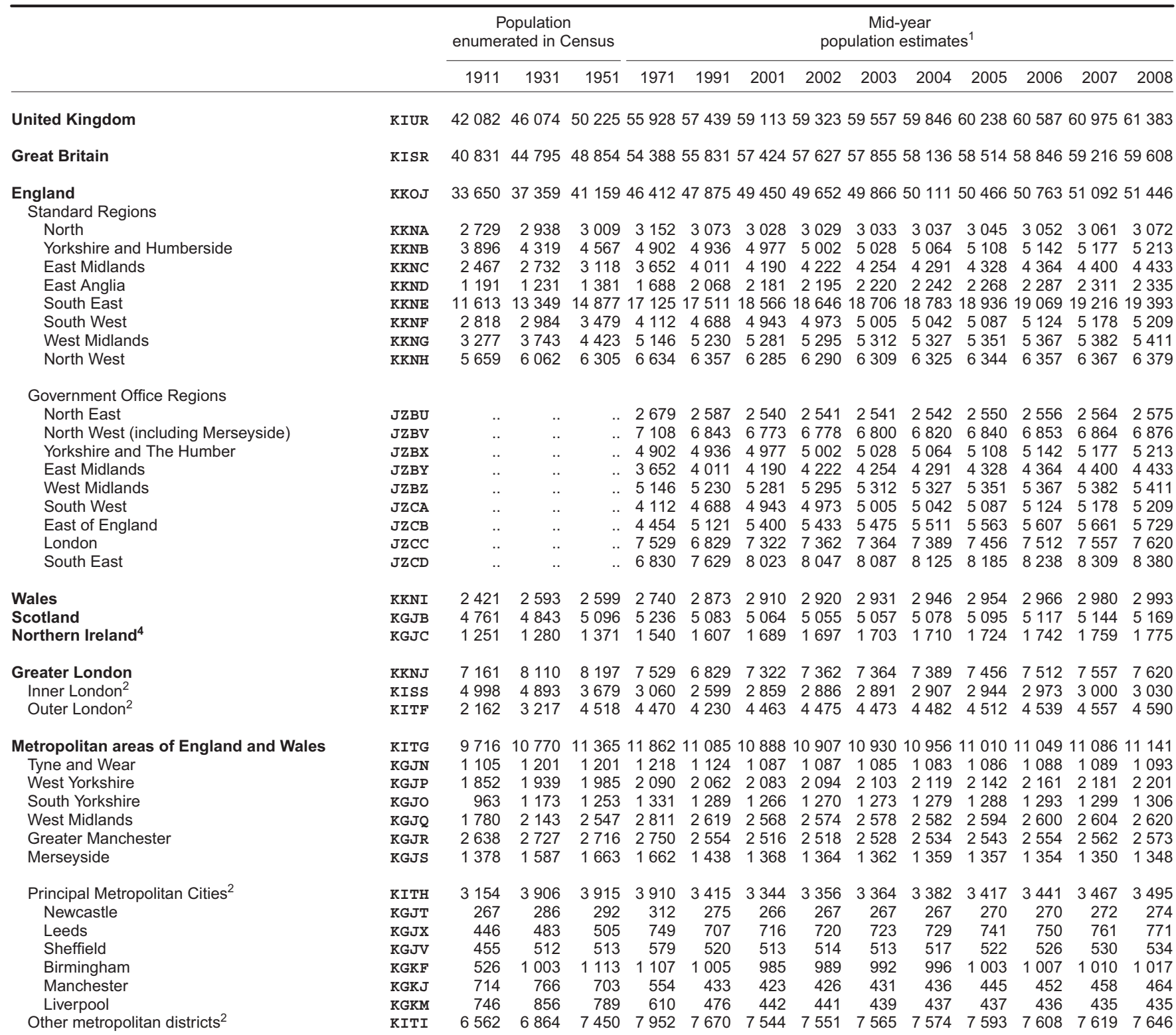

Non-metropolitan districts of England and Wales KITJ 19194210722419629761328343123931383315723176632000322013244932685 Non-metropolitan cities ${ }^{2}$ ncl. Kingston-upon-Hull

Leicester

Nottingham

Bristol

Plymouth

Stoke-on-Trent

Cardiff

Newport

Industrial districts ${ }^{2,3}$

New Towns ${ }^{2,3}$

Resort, port and retirement districts ${ }^{2,3}$

Urban and mixed urban/rural districts ${ }^{2,3}$

Remoter, mainly rural districts ${ }^{2,3}$

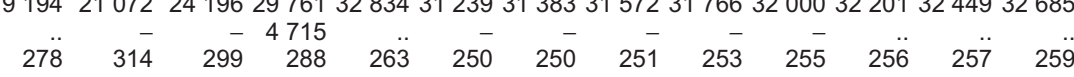

$\begin{array}{llllllllllllll}\text { KKNZ } & 278 & 314 & 299 & 288 & 263 & 250 & 250 & 251 & 253 & 255 & 256 & 257 & 259 \\ \text { KKOA } & 227 & 239 & 285 & 285 & 281 & 283 & 282 & 282 & 283 & 286 & 290 & 293 & 295\end{array}$

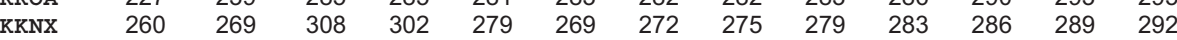

$\begin{array}{llllllllllllll}\text { KKNX } & 260 & 269 & 308 & 302 & 279 & 269 & 272 & 275 & 279 & 283 & 286 & 289 & 292 \\ \text { KKNV } & 357 & 397 & 443 & 433 & 392 & 390 & 391 & 394 & 397 & 406 & 410 & 416 & 421\end{array}$

$\begin{array}{llllllllllllll}\text { KKNV } & 357 & 397 & 443 & 433 & 392 & 390 & 391 & 394 & 397 & 406 & 410 & 416 & 421 \\ \text { KITL } & 207 & 215 & 225 & 249 & 251 & 241 & 242 & 242 & 244 & 246 & 248 & 251 & 253\end{array}$

$\begin{array}{llllllllllllll}\text { KITL } & 207 & 215 & 225 & 249 & 251 & 241 & 242 & 242 & 244 & 246 & 248 & 251 & 253 \\ \text { KKOD } & 235 & 277 & 275 & 265 & 249 & 240 & 239 & 239 & 239 & 239 & 240 & 239 & 240\end{array}$

$\begin{array}{llllllllllllll}\text { KKOB } & 182 & 224 & 244 & 291 & 297 & 310 & 311 & 310 & 312 & 314 & 318 & 321 & 325\end{array}$

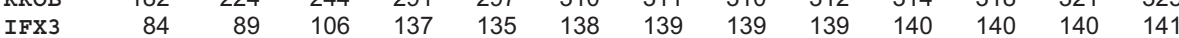

City of Edinburgh local government district City of Glasgow local government district

Belfast $^{4}$

$\begin{array}{lrrrrrrrrrrrrr}\text { IFX3 } & 84 & 89 & 106 & 137 & 135 & 138 & 139 & 139 & 139 & 140 & 140 & 140 & 141 \\ \text { KITM } & . & - & - & 6486 & & - & - & - & - & - & - & - & \end{array}$

1 Mid-2002 to mid-2005 population estimates for the UK and England \& 41931 figures shown for Northern Ireland and the City of Belfast relate to the Wales have been updated to include the latest revised estimates that take 1937 Census.

into account improved estimates of international migration.

2 Details of the classification by broad area type are given in recent issues of the ONS annual reference volume Key Population and Vital Statistics; local and health authority areas (Series VS). The ten broad area types include all local authorities in England and Wales.

3 The breakdown of non-metropolitan districts by area type has not been provided from mid-2001 onwards. This is because the effect of boundary changes due to the major local government reorganisation on 1 April 1995 and 1 April 1996 (particularly in Wales) make the comparison of 2001 data with data for earlier years invalid.

Sources: Office for National Statistics: 01329813318 General Register Office for Scotland; Northern Ireland Statistics and Research Agency 


\begin{tabular}{|c|c|c|c|c|c|c|c|c|c|c|c|c|}
\hline & 0 to 4 & 5 to 9 & 10 to 14 & 15 to 19 & 20 to 24 & 25 to 29 & 30 to 34 & 35 to 44 & 45 to 59 & 60 to 74 & $\begin{array}{r}75 \text { and } \\
\text { over }\end{array}$ & $\begin{array}{r}\text { All ages } \\
(=100 \%) \\
\text { (thousands) }\end{array}$ \\
\hline \multicolumn{13}{|l|}{ White $^{1}$} \\
\hline British & 6 & 5 & 6 & 6 & 6 & 6 & 6 & 15 & 20 & 16 & 8 & 49139 \\
\hline Other & 5 & 4 & 4 & 4 & 9 & 13 & 11 & 17 & 17 & 12 & 6 & 3188 \\
\hline \multicolumn{13}{|l|}{ Mixed } \\
\hline White and Black Caribbean & 21 & 16 & 16 & 14 & 9 & 6 & 4 & 8 & 4 & 1 & 1 & 241 \\
\hline White and Black African & 24 & 16 & 11 & 9 & 9 & 5 & 5 & 10 & 8 & 2 & 0 & 98 \\
\hline Other Mixed & 15 & 12 & 10 & 11 & 9 & 10 & 8 & 13 & 7 & 3 & 1 & 151 \\
\hline \multicolumn{13}{|l|}{ Asian } \\
\hline Indian & 7 & 6 & 6 & 7 & 8 & 11 & 11 & 15 & 18 & 9 & 3 & 1245 \\
\hline Pakistani & 13 & 11 & 9 & 9 & 9 & 10 & 9 & 15 & 10 & 4 & 1 & 995 \\
\hline Bangladeshi & 14 & 10 & 10 & 7 & 10 & 11 & 12 & 13 & 8 & 4 & 1 & 364 \\
\hline Other Asian & 8 & 7 & 6 & 7 & 8 & 11 & 12 & 19 & 15 & 5 & 2 & 501 \\
\hline \multicolumn{13}{|l|}{ Black } \\
\hline Black Other & 15 & 9 & 11 & 8 & 9 & 8 & 5 & 18 & 10 & 6 & 1 & 83 \\
\hline Chinese & 4 & 4 & 3 & 7 & 16 & 14 & 11 & 16 & 19 & 4 & 2 & 255 \\
\hline Other & 8 & 6 & 6 & 7 & 10 & 12 & 11 & 19 & 14 & 5 & 2 & 903 \\
\hline$A \|^{2}$ & 6 & 6 & 6 & 6 & 7 & 7 & 6 & 15 & 19 & 14 & 7 & 60554 \\
\hline
\end{tabular}

1 Respondents in Northern Ireland who state that their ethnicity is white are 2 Includes those who did not state their ethnic origin and those in Northern Irenot asked this question.

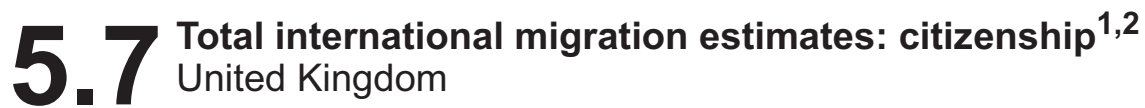

Citizenship by country of next or last residence

Thousands $^{3}$

\begin{tabular}{|c|c|c|c|c|c|c|c|}
\hline & \multirow[b]{2}{*}{ All citizenships } & \multirow[b]{2}{*}{ British } & \multirow[b]{2}{*}{ Non-British } & \multirow{2}{*}{$\begin{array}{c}\text { European } \\
\text { Union }^{4}\end{array}$} & \multicolumn{2}{|c|}{ Commonwealth } & \multirow[b]{2}{*}{ Other foreign ${ }^{6}$} \\
\hline & & & & & Old & $\mathrm{New}^{5}$ & \\
\hline \multicolumn{8}{|c|}{ Inflow } \\
\hline & C58E & $\mathrm{C} 58 \mathrm{H}$ & C58K & C58N & C58Q & C58T & C58W \\
\hline 2000 & 479 & 99 & 379 & 63 & 56 & 91 & 169 \\
\hline 2001 & 479 & 110 & 370 & 57 & 65 & 84 & 164 \\
\hline 2002 & 513 & 97 & 416 & 59 & 63 & 92 & 201 \\
\hline 2003 & 508 & 99 & 409 & 64 & 62 & 105 & 177 \\
\hline 2004 & 586 & 88 & 498 & 128 & 73 & 141 & 155 \\
\hline 2005 & 563 & 96 & 466 & 149 & 62 & 117 & 137 \\
\hline 2006 & 591 & 81 & 510 & 167 & 62 & 139 & 142 \\
\hline 2007 & 577 & 75 & 502 & 197 & 45 & 130 & 131 \\
\hline \multicolumn{8}{|c|}{ Outflow } \\
\hline & C58F & C58I & C58L & C58O & C58R & C58U & C58X \\
\hline 2000 & 321 & 161 & 160 & 57 & 32 & 15 & 55 \\
\hline 2001 & 306 & 158 & 149 & 49 & 32 & 19 & 49 \\
\hline 2002 & 358 & 185 & 174 & 52 & 42 & 16 & 64 \\
\hline 2004 & 342 & 195 & 147 & 42 & 33 & 19 & 52 \\
\hline 2005 & 359 & 185 & 174 & 54 & 37 & 23 & 59 \\
\hline 2006 & 400 & 207 & 194 & 66 & 42 & 24 & 61 \\
\hline 2007 & 340 & 171 & 169 & 68 & 31 & 26 & 43 \\
\hline \multicolumn{8}{|c|}{ Balance } \\
\hline & C58G & C58J & C58M & C58P & C58S & C58V & C58Y \\
\hline 2000 & 158 & -62 & 220 & 6 & 24 & 76 & 114 \\
\hline 2001 & 173 & -48 & 221 & 8 & 33 & 65 & 115 \\
\hline 2002 & 154 & -87 & 242 & 7 & 21 & 77 & 137 \\
\hline 2003 & 147 & -91 & 238 & 14 & 20 & 88 & 115 \\
\hline 2004 & 244 & -107 & 351 & 85 & 40 & 122 & 104 \\
\hline 2005 & 204 & -89 & 293 & 95 & 25 & 94 & 78 \\
\hline 2006 & 191 & -126 & 316 & 100 & 20 & 115 & 81 \\
\hline 2007 & 237 & -96 & 333 & 128 & 13 & 103 & 88 \\
\hline
\end{tabular}

1 The1998-2005 were revised, following changes to the weightings used to 4 European Union estimates are for the EU15 (Austria, Belgium, Denmark, Fingross up the IPS data, in November 2007. Therefore they may not agree with previously published estimates.

2 Based mainly on data from the IPS. Includes adjustments for (1) those whose intended length of stay changes so that their migrant status changes; (2) asylum seekers and their dependants not identified by the IPS; and (3) flows between the UK and the Republic of Ireland.

European Union estimates are for the EU15 (Austria, Belgium, Denmark, Fin-
land, France, Germany, Greece, Republic of Ireland, Italy, Luxembourg, Netherlands, Portugal, Spain and Sweden) from 1998- 2003, EU25 (EU15 and Czech Republic, Estonia, Hungary, Latvia, Lithuania, Malta, Poland,Cyprus, Slovakia and Slovenia). from 2004-2006, and for the EU27 (EU25 plus Bulgaria and Romania) from 2007. British citizens are excluded from all EU citizenship groupings and are shown separately.

3 Estimates of international migration flows are shown rounded to the nearest 5 For 2004 onwards, the New Commonwealth excludes Malta and Cyprus.

thousand, rather than nearest hundred, as they are considered less reliable 6 For 2004 onwards, Other foreign excludes the eight Central and Eastern Euroat the more detailed level. 
Population and vital statistics

5 . $\begin{aligned} & \text { Estimates of migration } \\ & \text { occupation }\end{aligned}$

Thousands

\begin{tabular}{|c|c|c|c|c|c|c|c|c|c|c|c|c|}
\hline & \multicolumn{3}{|c|}{ Total } & \multicolumn{3}{|c|}{ Professional and managerial } & \multicolumn{3}{|c|}{ Manual and clerical } & \multicolumn{3}{|c|}{ Not gainfully employed ${ }^{3}$} \\
\hline & Persons & Males & Females & Persons & Males & Females & Persons & Males & Females & Persons & Males & Females \\
\hline \multicolumn{13}{|c|}{ Inflow } \\
\hline & KGOA & KGOB & KGOC & KGOD & KGOE & KGOF & KGOG & $\mathrm{KGOH}$ & KGOI & KGOJ & KGOK & KGOL \\
\hline 1991 & 255 & 117 & 138 & 76 & 47 & 28 & 53 & 22 & 31 & 127 & 48 & 79 \\
\hline 1992 & 207 & 95 & 112 & 59 & 37 & 22 & 42 & 16 & 26 & 106 & 42 & 65 \\
\hline 1993 & 204 & 97 & 108 & 63 & 40 & 23 & 41 & 20 & 21 & 100 & 37 & 63 \\
\hline 1994 & 243 & 121 & 121 & 79 & 47 & 32 & 54 & 30 & 24 & 110 & 44 & 65 \\
\hline 1995 & 235 & 125 & 111 & 83 & 55 & 28 & 44 & 19 & 25 & 108 & 51 & 57 \\
\hline 1996 & 261 & 124 & 137 & 86 & 51 & 35 & 55 & 24 & 31 & 120 & 50 & 71 \\
\hline 1997 & 273 & 137 & 136 & 89 & 57 & 33 & 42 & 23 & 19 & 141 & 57 & 84 \\
\hline 1998 & 318 & 160 & 158 & 112 & 65 & 47 & 71 & 35 & 35 & 136 & 60 & 76 \\
\hline 1999 & 354 & 181 & 173 & 131 & 77 & 54 & 75 & 40 & 35 & 148 & 63 & 84 \\
\hline 2000 & 359 & 188 & 171 & 162 & 98 & 64 & 64 & 33 & 31 & 133 & 57 & 76 \\
\hline 2001 & 372 & 187 & 185 & 138 & 77 & 62 & 77 & 39 & 38 & 157 & 72 & 85 \\
\hline 2002 & 386 & 200 & 186 & 139 & 78 & 62 & 83 & 45 & 38 & 163 & 77 & 87 \\
\hline 2003 & 427 & 211 & 215 & 146 & 76 & 71 & 92 & 46 & 46 & 189 & 90 & 99 \\
\hline 2004 & 518 & 261 & 257 & 175 & 102 & 73 & 132 & 66 & 65 & 212 & 92 & 119 \\
\hline 2005 & 496 & 273 & 223 & 168 & 97 & 71 & 145 & 90 & 55 & 184 & 86 & 98 \\
\hline 2006 & 529 & 280 & 249 & 154 & 82 & 72 & 136 & 79 & 57 & 239 & 120 & 119 \\
\hline 2007 & 527 & 286 & 241 & 168 & 99 & 69 & 136 & 79 & 57 & 223 & 108 & 115 \\
\hline \multicolumn{13}{|c|}{ Outflow } \\
\hline & KGPA & KGPB & KGPC & KGPD & KGPE & KGPF & KGPG & KGPH & KGPI & KGPJ & KGPK & KGPL \\
\hline 1991 & 247 & 124 & 123 & 87 & 50 & 37 & 51 & 29 & 21 & 110 & 46 & 64 \\
\hline 1992 & 235 & 117 & 118 & 85 & 51 & 33 & 49 & 24 & 25 & 101 & 41 & 60 \\
\hline 1993 & 223 & 117 & 106 & 73 & 39 & 33 & 46 & 25 & 21 & 105 & 53 & 52 \\
\hline 1994 & 197 & 96 & 102 & 57 & 33 & 24 & 50 & 24 & 26 & 90 & 38 & 52 \\
\hline 1995 & 198 & 105 & 93 & 64 & 43 & 21 & 43 & 23 & 20 & 91 & 39 & 52 \\
\hline 1996 & 223 & 109 & 114 & 87 & 54 & 32 & 48 & 23 & 24 & 89 & 31 & 58 \\
\hline 1997 & 232 & 125 & 107 & 88 & 59 & 29 & 50 & 23 & 26 & 94 & 43 & 51 \\
\hline 1998 & 206 & 103 & 103 & 82 & 48 & 34 & 42 & 22 & 21 & 82 & 33 & 48 \\
\hline 1999 & 245 & 132 & 114 & 97 & 60 & 38 & 69 & 32 & 37 & 79 & 41 & 39 \\
\hline 2000 & 278 & 154 & 124 & 128 & 80 & 48 & 59 & 36 & 23 & 90 & 37 & 53 \\
\hline 2001 & 250 & 135 & 115 & 102 & 66 & 36 & 60 & 30 & 30 & 88 & 39 & 49 \\
\hline 2002 & 305 & 161 & 144 & 123 & 79 & 45 & 80 & 40 & 40 & 102 & 42 & 59 \\
\hline 2003 & 314 & 165 & 149 & 108 & 60 & 49 & 103 & 59 & 44 & 102 & 46 & 57 \\
\hline 2004 & 310 & 152 & 158 & 114 & 65 & 49 & 73 & 40 & 33 & 123 & 47 & 76 \\
\hline 2005 & 328 & 187 & 141 & 137 & 88 & 50 & 83 & 47 & 37 & 108 & 53 & 55 \\
\hline 2006 & 369 & 208 & 162 & 125 & 77 & 48 & 119 & 73 & 46 & 126 & 58 & 68 \\
\hline 2007 & 318 & 178 & 139 & 112 & 65 & 48 & 101 & 60 & 42 & 104 & 54 & 50 \\
\hline
\end{tabular}

Balance

\begin{tabular}{|c|c|c|c|c|c|c|c|c|c|c|c|c|}
\hline & KGRA & KGRB & KGRC & KGRD & KGRE & KGRF & KGRG & $\mathrm{KGRH}$ & KGRI & KGRJ & KGRK & KGRL \\
\hline 1991 & 8 & -7 & 16 & -11 & -3 & -8 & 2 & -8 & 10 & 17 & 3 & 14 \\
\hline 1992 & -28 & -22 & -6 & -26 & -14 & -12 & -7 & -8 & 1 & 5 & - & 5 \\
\hline 1993 & -19 & -20 & 2 & -9 & - & -10 & -5 & -5 & - & -4 & -15 & 11 \\
\hline 1994 & 45 & 26 & 20 & 22 & 14 & 8 & 4 & 6 & -2 & 20 & 6 & 13 \\
\hline 1995 & 37 & 19 & 18 & 18 & 11 & 7 & 1 & -4 & 6 & 17 & 12 & 5 \\
\hline 1996 & 37 & 15 & 22 & -1 & -3 & 2 & 7 & - & 7 & 31 & 18 & 13 \\
\hline 1997 & 40 & 12 & 29 & 1 & -2 & 3 & -7 & -1 & -7 & 47 & 14 & 33 \\
\hline 1998 & 113 & 57 & 55 & 30 & 17 & 13 & 28 & 13 & 15 & 54 & 27 & 27 \\
\hline 2000 & 82 & 35 & 47 & 34 & 18 & 16 & 5 & -3 & 8 & 43 & 20 & 23 \\
\hline 2001 & 122 & 52 & 70 & 36 & 11 & 25 & 17 & 9 & 8 & 70 & 33 & 36 \\
\hline 2002 & 81 & 39 & 42 & 16 & -1 & 17 & 3 & 5 & -2 & 62 & 34 & 27 \\
\hline 2003 & 113 & 47 & 67 & 38 & 16 & 22 & -11 & -13 & 2 & 86 & 44 & 42 \\
\hline 2004 & 208 & 109 & 99 & 61 & 37 & 24 & 58 & 26 & 32 & 89 & 45 & 43 \\
\hline 2005 & 168 & 86 & 82 & 31 & 9 & 21 & 61 & 43 & 18 & 76 & 34 & 43 \\
\hline 2006 & 160 & 72 & 87 & 29 & 5 & 24 & 17 & 6 & 11 & 113 & 62 & 51 \\
\hline 2007 & 209 & 108 & 101 & 55 & 34 & 21 & 34 & 19 & 15 & 119 & 54 & 65 \\
\hline
\end{tabular}

1 See chapter text.

2 The 1991-2005 estimates were revised, following changes to the weight-

ings used to gross up the IPS data, in November 2007. Therefore the above

figures may not agree with estimates published before then.

Source: Office for National Statistics: 01329444645

3 Includes housewives, students, children and retired persons. 


\section{Estimates of migration into and out of the United Kingdom ${ }^{1}$ by citizenship and country of last or next residence}

Thousands

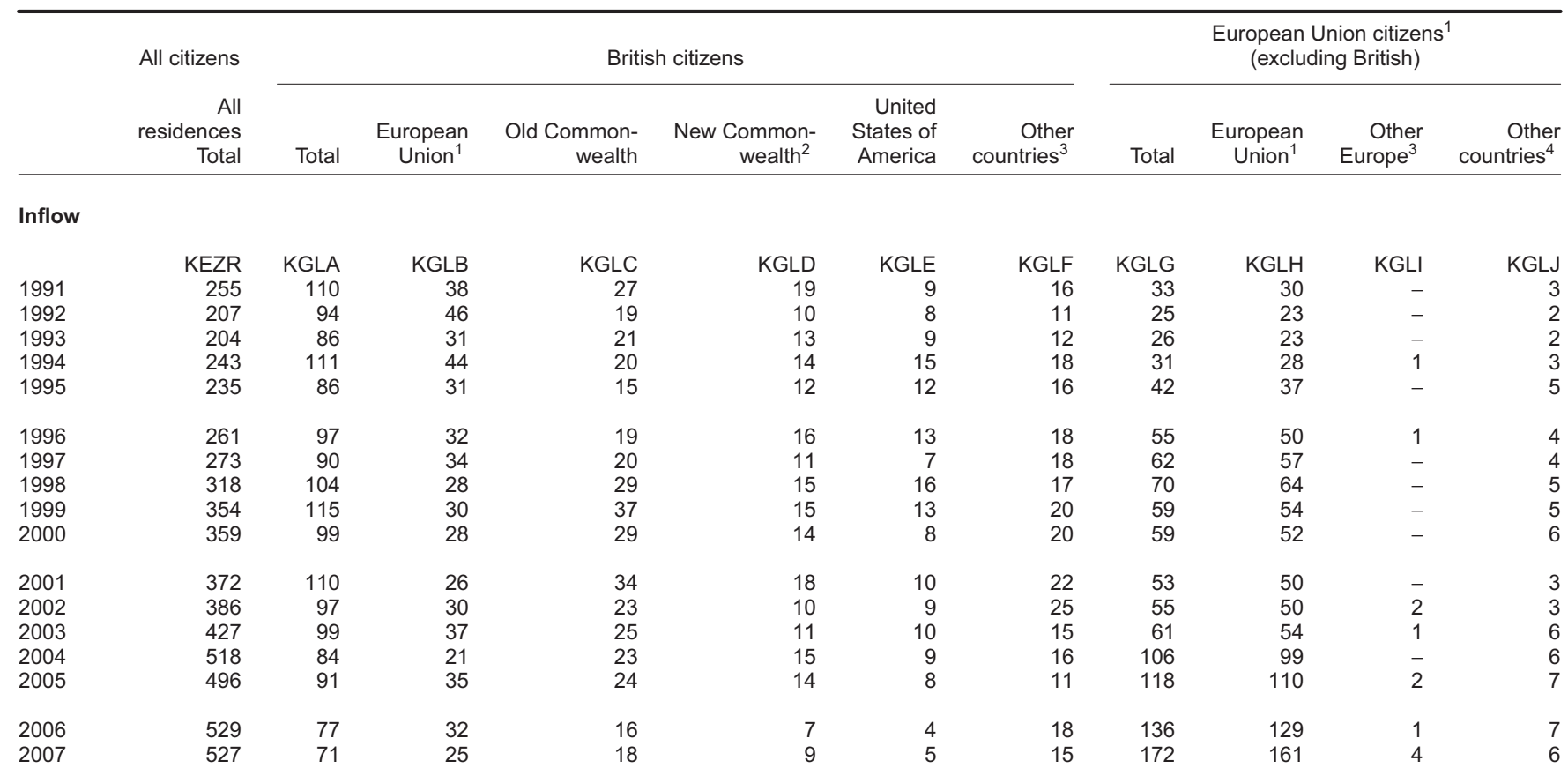

Outflow

\begin{tabular}{|c|c|c|c|c|c|c|c|c|c|c|c|}
\hline & KEZS & KGMA & KGMB & KGMC & KGMD & KGME & KGMF & KGMG & $\mathrm{KGMH}$ & KGMI & KGMJ \\
\hline 1991 & 247 & 141 & 47 & 43 & 16 & 13 & 22 & 32 & 23 & 2.0 & 6 \\
\hline 1992 & 235 & 137 & 45 & 35 & 13 & 17 & 27 & 17 & 14 & - & 3 \\
\hline 1993 & 223 & 130 & 45 & 35 & 12 & 17 & 21 & 24 & 21 & - & 3 \\
\hline 1994 & 197 & 111 & 35 & 29 & 11 & 15 & 21 & 23 & 19 & - & 4 \\
\hline 1995 & 198 & 122 & 39 & 35 & 10 & 18 & 20 & 20 & 16 & - & 4 \\
\hline 1996 & 223 & 143 & 53 & 38 & 16 & 16 & 20 & 24 & 18 & - & 6 \\
\hline 1997 & 232 & 135 & 41 & 38 & 13 & 16 & 27 & 32 & 27 & 1.0 & 4 \\
\hline 1998 & 206 & 114 & 37 & 36 & 8 & 15 & 19 & 26 & 21 & 1.0 & 4 \\
\hline 2000 & 278 & 141 & 41 & 48 & 9 & 19 & 24 & 46 & 39 & 1.0 & 6 \\
\hline 2001 & 250 & 133 & 41 & 47 & 7 & 15 & 22 & 40 & 34 & 1.0 & 5 \\
\hline 2002 & 305 & 164 & 68 & 44 & 10 & 18 & 24 & 42 & 38 & - & 4 \\
\hline 2003 & 314 & 170 & 71 & 55 & 9 & 13 & 22 & 42 & 33 & 3.0 & 5 \\
\hline 2004 & 310 & 184 & 68 & 62 & 12 & 16 & 27 & 34 & 30 & 1.0 & 3 \\
\hline 2005 & 328 & 174 & 74 & 62 & 6 & 10 & 22 & 47 & 42 & 1.0 & 4 \\
\hline 2006 & 369 & 196 & 71 & 69 & 10 & 16 & 30 & 59 & 52 & - & \\
\hline 2007 & 318 & 159 & 54 & 60 & 7 & 12 & 27 & 65 & 57 & 2.0 & \\
\hline
\end{tabular}

Balance

\begin{tabular}{|c|c|c|c|c|c|c|c|c|c|c|c|}
\hline & KEZT & KGNA & KGNB & KGNC & KGND & KGNE & KGNF & KGNG & $\mathrm{KGNH}$ & KGNI & KGNJ \\
\hline 1991 & 8 & -30 & -8 & -16 & 3 & -3 & -5 & - & 6 & -2 & -3 \\
\hline 1992 & -28 & -43 & 1 & -16 & -3 & -9 & -16 & 8 & 10 & - & -1 \\
\hline 1993 & -19 & -44 & -14 & -14 & 1 & -8 & -9 & 2 & 3 & - & - \\
\hline 1994 & 45 & - & 10 & -10 & 2 & - & -3 & 8 & 10 & 1 & -2 \\
\hline 1996 & 37 & -46 & -21 & -20 & - & -3 & -3 & 31 & 32 & 1 & -1 \\
\hline 1997 & 40 & -45 & -7 & -18 & -2 & -9 & -9 & 30 & 30 & -1 & 1 \\
\hline 1998 & 113 & -10 & -10 & -6 & 7 & 1 & -2 & 44 & 43 & -1 & 1 \\
\hline 2000 & 82 & -42 & -14 & -19 & 6 & -11 & -3 & 13 & 14 & -1 & - \\
\hline 2001 & 122 & -23 & -15 & -13 & 11 & -5 & - & 13 & 16 & -1 & -2 \\
\hline 2002 & 81 & -66 & -37 & -21 & - & -9 & 1 & 13 & 11 & 2 & -1 \\
\hline 2003 & 113 & -70 & -34 & -29 & 2 & -2 & -7 & 19 & 21 & -3 & 1 \\
\hline 2004 & 208 & -100 & -47 & -39 & 3 & -7 & -11 & 72 & 69 & -1 & 3 \\
\hline 2005 & 168 & -83 & -39 & -38 & 8 & -2 & -12 & 72 & 67 & 1 & 3 \\
\hline 2006 & 160 & -119 & -39 & -53 & -3 & -12 & -12 & 78 & 78 & 1 & - \\
\hline 2007 & 209 & -88 & -29 & -42 & 2 & -7 & -12 & 107 & 104 & 2 & 1 \\
\hline
\end{tabular}

The 1991-2005 estimates were revised following changes to the weight- 2 From 2004 onwards, the New Commonwealth excludes Malta and Cyprus. ings used to gross up the IPS data, in November 2007. Therefore the above 3 From 2004 onwards these categories exclude the A8 Central and Eastern figures may not agree with previous estimates published before then. countries that joined the EU in 2004. From 2007 these categories exclude the 1 EU estimates are for the EU15 (Austria, Belgium, Denmark, Finland, A2 countries (Bulgaria and Romania) that joined the EU in 2007. France, Germany, Greece, the Irish Republic, Italy, Luxembourg, Nether- 4 From 2004 onwards Other countries excludes Malta and cyprus. lands, Portugal, Spain and Sweden) from 1991-2003, EU25 (EU15 and A8 groupings plus Malta and Cyprus) form 2004 -2006 and for the EU27 (EU25 plus Bulgaria and Romania) from 2007. 
Population and vital statistics

\begin{tabular}{|c|c|c|c|c|c|c|c|c|c|c|c|c|c|}
\hline & \multicolumn{8}{|c|}{ Commonwealth $^{1}$ citizens } & \multicolumn{5}{|c|}{ Other foreign citizens ${ }^{3}$} \\
\hline & Total & $\begin{array}{r}\text { Aust- } \\
\text { ralia }\end{array}$ & Canada & $\begin{array}{r}\text { New } \\
\text { Zealand }\end{array}$ & $\begin{array}{l}\text { South } \\
\text { Africa }\end{array}$ & $\begin{array}{r}\text { Indian }{ }^{2} \\
\text { sub- } \\
\text { continent }\end{array}$ & $\begin{array}{r}\text { Other } \\
\text { African } \\
\text { Common- } \\
\text { wealth }\end{array}$ & $\begin{array}{l}\text { Other } \\
\text { count- } \\
\text { ries }\end{array}$ & Total & $\begin{array}{c}\text { Euro- } \\
\text { pean } \\
\text { Union }^{4}\end{array}$ & $\begin{array}{c}\text { Other } \\
\text { Europe }^{5}\end{array}$ & $\begin{array}{r}\text { United } \\
\text { States of } \\
\text { America }\end{array}$ & $\begin{array}{c}\text { Other } \\
\text { coun- } \\
\text { tries }\end{array}$ \\
\hline \multicolumn{14}{|c|}{ Inflow } \\
\hline & KGLK & KGLL & KGLM & KGLN & KTDK & $\mathrm{IBH} 3$ & KGLQ & $\mathrm{IBH} 4$ & KGLU & KGLV & KGLW & KGLX & KGLY \\
\hline 1991 & 61 & 11 & 4 & 6 & 1 & 16 & 8 & 15 & 51 & 6 & 7 & 14 & 26 \\
\hline 1992 & 47 & 9 & 2 & 6 & - & 12 & 6 & 13 & 41 & 2 & 7 & 9 & 23 \\
\hline 1993 & 48 & 10 & 3 & 5 & 2 & 13 & 5 & 10 & 44 & 2 & 11 & 11 & 19 \\
\hline 1994 & 48 & 9 & 2 & 6 & 1 & 10 & 8 & 13 & 52 & 5 & 14 & 11 & 22 \\
\hline 1995 & 56 & 12 & 5 & 6 & 2 & 11 & 4 & 16 & 52 & 2 & 10 & 11 & 29 \\
\hline 1996 & 59 & 14 & 3 & 7 & 4 & 15 & 6 & 11 & 49 & - & 7 & 15 & 27 \\
\hline 1997 & 75 & 14 & 5 & 7 & 5 & 20 & 6 & 20 & 45 & - & 7 & 11 & 27 \\
\hline 1998 & 88 & 24 & 5 & 13 & 11 & 13 & 10 & 12 & 56 & 4 & 7 & 18 & 27 \\
\hline 1999 & 101 & 27 & 2 & 12 & 12 & 22 & 14 & 12 & 79 & 3 & 18 & 15 & 43 \\
\hline 2001 & 120 & 30 & 4 & 9 & 13 & 31 & 17 & 17 & 89 & 1 & 13 & 12 & 63 \\
\hline 2002 & 121 & 20 & 4 & 9 & 20 & 33 & 22 & 11 & 113 & 2 & 11 & 16 & 84 \\
\hline 2003 & 142 & 21 & 5 & 7 & 20 & 46 & 24 & 18 & 124 & 2 & 22 & 17 & 84 \\
\hline 2004 & 204 & 24 & 5 & 8 & 30 & 77 & 38 & 21 & 125 & 1 & 12 & 14 & 98 \\
\hline 2005 & 172 & 22 & 4 & 11 & 23 & 74 & 24 & 13 & 115 & 3 & 13 & 14 & 85 \\
\hline 2006 & 193 & 29 & 6 & 8 & 16 & 95 & 17 & 21 & 122 & 9 & 13 & 15 & 86 \\
\hline 2007 & 169 & 16 & 4 & 8 & 13 & 89 & 17 & 21 & 115 & 5 & 11 & 15 & 84 \\
\hline
\end{tabular}

Outflow

\begin{tabular}{|c|c|c|c|c|c|c|c|c|c|c|c|c|c|}
\hline & KGMK & KGML & KGMM & KGMN & KTDL & IBH5 & KGMQ & $\mathrm{IBH} 7$ & KGMU & KGMV & KGMW & KGMX & KGMY \\
\hline 1991 & 33 & 7 & 4 & 5 & - & 4 & 3 & 10 & 41 & 2 & 3 & 17 & 2 \\
\hline 1992 & 28 & 6 & 2 & 5 & 1 & 3 & 3 & 8 & 53 & 1 & 12 & 20 & \\
\hline 1993 & 32 & 8 & 2 & 4 & 1 & 4 & 4 & 8 & 38 & 2 & 3 & 17 & \\
\hline 1994 & 28 & 6 & 2 & 4 & 1 & 4 & 2 & 9 & 35 & 1 & 10 & 8 & \\
\hline 1996 & 29 & 8 & 2 & 3 & 2 & 3 & 2 & 10 & 27 & 1 & 7 & 5 & \\
\hline 1997 & 36 & 7 & 1 & 5 & 5 & 4 & 2 & 13 & 29 & 3 & 5 & 9 & \\
\hline 1998 & 30 & 9 & 1 & 3 & 4 & 3 & 2 & 7 & 35 & 2 & 7 & 9 & \\
\hline 2000 & 43 & 12 & 3 & 8 & 5 & 3 & 2 & 10 & 48 & 3 & 11 & 9 & \\
\hline 2001 & 44 & 15 & 3 & 6 & 5 & 4 & 2 & 9 & 33 & 1 & 9 & 7 & \\
\hline 2002 & 52 & 18 & 6 & 9 & 5 & 5 & 2 & 7 & 47 & 2 & 12 & 16 & 18 \\
\hline 2003 & 53 & 19 & 2 & 8 & 9 & 5 & 2 & 8 & 48 & 2 & 12 & 8 & \\
\hline 2004 & 50 & 17 & 4 & 5 & 7 & 4 & 3 & 12 & 42 & 5 & 2 & 8 & \\
\hline 2005 & 59 & 15 & 4 & 8 & 9 & 13 & 2 & 7 & 49 & 1 & 7 & 14 & \\
\hline 2006 & 65 & 17 & 5 & 7 & 13 & 11 & 3 & 8 & 50 & 3 & 7 & 11 & \\
\hline 2007 & 56 & 17 & 2 & 5 & 9 & 15 & 3 & 6 & 37 & 4 & 5 & 6 & \\
\hline
\end{tabular}

Balance

\begin{tabular}{|c|c|c|c|c|c|c|c|c|c|c|c|c|c|}
\hline & KGNK & KGNL & KGNM & KGNN & KTDM & IBH6 & KGNQ & $\mathrm{IBH} 8$ & KGNU & KGNV & KGNW & KGNX & KGNY \\
\hline 1991 & 28 & 5 & - & 2 & 1 & 12 & 5 & 5 & 10 & 4 & 4 & -3 & 6 \\
\hline 1992 & 19 & 3 & - & 1 & -1 & 9 & 3 & 4 & -13 & 1 & -5 & -11 & 2 \\
\hline 1993 & 16 & 2 & 1 & 1 & 1 & 8 & 2 & 2 & 7 & - & 9 & -5 & 3 \\
\hline 1994 & 20 & 3 & 1 & 2 & - & 5 & 6 & 3 & 17 & 4 & 4 & 3 & 7 \\
\hline 1995 & 29 & 5 & 4 & 2 & - & 9 & 2 & 6 & 22 & 2 & 5 & 2 & 13 \\
\hline 1996 & 30 & 7 & 1 & 4 & 2 & 12 & 4 & 1 & 22 & -1 & - & 10 & 13 \\
\hline 1997 & 39 & 7 & 4 & 2 & 1 & 16 & 4 & 7 & 16 & -2 & 2 & 3 & 14 \\
\hline 1998 & 59 & 15 & 3 & 10 & 7 & 10 & 8 & 6 & 21 & 2 & 1 & 9 & 9 \\
\hline 2000 & 70 & 10 & 4 & 2 & 9 & 26 & 10 & 8 & 41 & -1 & - & 4 & 38 \\
\hline 2001 & 76 & 15 & 1 & 3 & 8 & 27 & 14 & 8 & 56 & - & 3 & 6 & 47 \\
\hline 2002 & 69 & 2 & -1 & - & 15 & 29 & 21 & 4 & 66 & - & -1 & - & 66 \\
\hline 2003 & 89 & 2 & 3 & -1 & 11 & 41 & 23 & 10 & 76 & -1 & 10 & 9 & 57 \\
\hline 2004 & 153 & 7 & 1 & 3 & 23 & 74 & 35 & 10 & 83 & -4 & 10 & 7 & 71 \\
\hline 2005 & 113 & 7 & 1 & 3 & 14 & 61 & 22 & 7 & 66 & 2 & 7 & 1 & 57 \\
\hline 2006 & 128 & 13 & 2 & 1 & 3 & 84 & 14 & 12 & 72 & 5 & 6 & 4 & 57 \\
\hline 2007 & 112 & - & 2 & 4 & 4 & 74 & 14 & 15 & 78 & 1 & 6 & 10 & 61 \\
\hline
\end{tabular}

The 1991 - 2005 estimates were revised following changes to the weight- 4 European Union estimates are for the EU15 ( Austria, Belgium, Denmark, Finings used to gross up the IPS data, in November 2007. Therefore the above land, France, Germany, Greece, the Irish Republic, Italy, Luxembourg, Netherdata may not agree with previous estimates published before then.

1 From 2004 onwards, the Commonwealth excludes Malta and Cyprus. lands, Portugal, Spain and Sweden) from 1991-2003, EU25 (EU15 and A8 groupings plus Malta and Cyprus) from 2004-2006 and for the EU27 (EU25 plus Bulgaria and Romania) from 2007.
From 2004 onwards Other Europe excludes the 8 Central and Eastern

2 Indian sub-contitent consists of Bangladesh, India, Sir Lanka and Pakistan.

3 From 2004 onwards Other foreign citizens excludes the A8 Central and European countries that joined the EU in 2004. From 2007 these categories Lithuania, Poland, Slovakia and Slovenia) that joined the EU in 2004. From exclude the A2 countries that joined the EU in 2007.

2007 Other foreign citizens exlcudes the A2 countries (Bulagria and Roma-

Source: Office for National Statistics: 01329444645 nia) that joined the EU in 2007. 


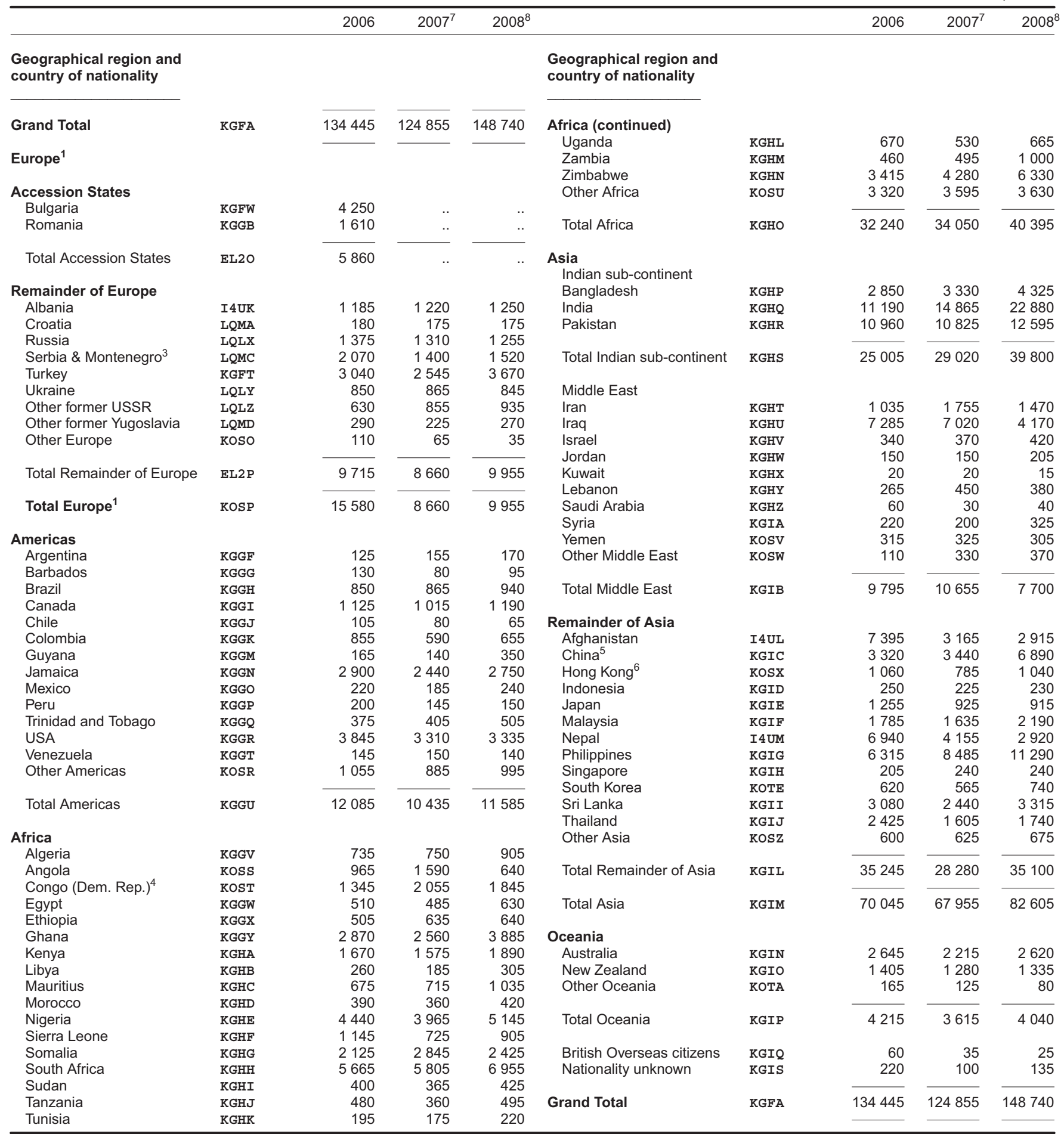

1 Members of the European Economic Area prior to 2005 and Swiss nation- 4 Formerly known as Zaire.

als are excluded throughout the period covered. 5 Includes Taiwan.

2 Data also excludes dependants of EEA and Swiss nationals in confirmed 6 Hong Kong (Special Administrative Region of China) includes British overseas relationships granted permanent residence.

territories citizens and stateless persons from Hong Kong and British Nationals

3 Serbia and Montenegro continue to be counted together due to the use of a (overseas).

single (Federal Republic of Yugoslavia) passport. $\quad 7$ Excludes natio

8 Provisional. 
Population and vital statistics

Number of principal applicants

\begin{tabular}{|c|c|c|c|c|c|c|c|c|c|c|}
\hline & & $2000^{2}$ & 2001 & 2002 & 2003 & 2004 & 2005 & 2006 & 2007 & $2008^{5}$ \\
\hline \multicolumn{11}{|l|}{ Europe } \\
\hline Albania & LQME & 1490 & 1065 & 1150 & 595 & 295 & 175 & 155 & 165 & 160 \\
\hline Macedonia & PTDW & 65 & 755 & 310 & 60 & 15 & 5 & - & 25 & - \\
\hline Moldova & VQHP & 235 & 425 & 820 & 380 & 170 & 115 & 45 & 30 & 20 \\
\hline Russia & ZÃEQ & 1000 & 450 & 295 & 280 & 190 & 130 & 115 & 80 & 50 \\
\hline Serbia \& Montenegro ${ }^{3}$ & ZAFA & 6070 & 3230 & 2265 & 815 & 290 & 155 & 70 & .. & .. \\
\hline Turkey & KEAW & 3990 & 3695 & 2835 & 2390 & 1230 & 755 & 425 & 210 & 195 \\
\hline Ukraine & ZAER & 770 & 445 & 365 & 300 & 120 & 55 & 50 & 40 & 30 \\
\hline Other Former USSR & ZAES & 1050 & 485 & 615 & 520 & 315 & 265 & 220 & 155 & 180 \\
\hline Other Europe & ZAEU & 2230 & 210 & 130 & 70 & 35 & 30 & 35 & 95 & 95 \\
\hline Total Europe & KEAZ & 22880 & 14215 & 13235 & 6295 & 3025 & 1810 & 1210 & 825 & 740 \\
\hline \multicolumn{11}{|l|}{ Americas } \\
\hline Colombia & KEBZ & 505 & 365 & 420 & 220 & 120 & 70 & 60 & 30 & 25 \\
\hline Ecuador & KYDB & 445 & 255 & 315 & 150 & 35 & 10 & 15 & 10 & 15 \\
\hline Jamaica & PTDX & 310 & 525 & 1310 & 965 & 455 & 325 & 215 & 240 & 240 \\
\hline Total Americas & КECT & 1420 & 1315 & 2290 & 1560 & 740 & 505 & 385 & 390 & 405 \\
\hline \multicolumn{11}{|l|}{ Africa } \\
\hline Algeria & котв & 1635 & 1140 & 1060 & 550 & 490 & 255 & 225 & 260 & 345 \\
\hline Angola & $\mathrm{KECU}$ & 800 & 1015 & 1420 & 850 & 400 & 145 & 95 & 95 & 80 \\
\hline Burundi & PTDZ & 620 & 610 & 700 & 650 & 265 & 90 & 35 & 25 & 15 \\
\hline Cameroon & $\mathrm{VQHU}$ & 355 & 380 & 615 & 505 & 360 & 290 & 260 & 160 & 115 \\
\hline Congo & PTEA & 485 & 540 & 600 & 320 & 150 & 65 & 45 & 25 & 25 \\
\hline Dem. Rep. Congo & KEEH & 1030 & 1370 & 2215 & 1540 & 1475 & 1080 & 570 & 370 & 335 \\
\hline Eritrea & PTEC & 505 & 620 & 1180 & 950 & 1105 & 1760 & 2585 & 1810 & 2255 \\
\hline Ethiopia & $\mathrm{KECW}$ & 415 & 610 & 700 & 640 & 540 & 385 & 200 & 90 & 130 \\
\hline Gambia & DMMA & 50 & 65 & 130 & 95 & 100 & 90 & 110 & 100 & 125 \\
\hline Ghana & $\mathrm{KECX}$ & 285 & 190 & 275 & 325 & 355 & 230 & 130 & 120 & 140 \\
\hline Ivory Coast & DMLZ & 445 & 275 & 315 & 390 & 280 & 210 & 170 & 100 & 70 \\
\hline Kenya & котс & 455 & 305 & 350 & 220 & 145 & 100 & 95 & 115 & 150 \\
\hline Liberia & $\mathrm{C} 53 \mathrm{~K}$ & 55 & 115 & 450 & 740 & 405 & 175 & 50 & 40 & 20 \\
\hline Libya & GH5U & 155 & 140 & 200 & 145 & 160 & 125 & 90 & 45 & 45 \\
\hline Nigeria & $\mathrm{KECY}$ & 835 & 810 & 1125 & 1010 & 1090 & 1025 & 790 & 780 & 820 \\
\hline Rwanda & ZAEV & 760 & 530 & 655 & 260 & 75 & 40 & 20 & 15 & 20 \\
\hline Tanzania & DMMC & 60 & 80 & 40 & 30 & 20 & 20 & 15 & 20 & 25 \\
\hline Uganda & KEEG & 740 & 480 & 715 & 705 & 405 & 205 & 165 & 130 & 130 \\
\hline Zimbabwe & GRFS & 1010 & 2140 & 7655 & 3295 & 2065 & 1075 & 1650 & 1800 & 3165 \\
\hline Africa & PTEB & 720 & 665 & 970 & 985 & 1050 & 735 & 555 & 510 & 600 \\
\hline Total Africa & KEEJ & 18185 & 20840 & 29710 & 20605 & 15045 & 10885 & 10500 & 8630 & 10270 \\
\hline \multicolumn{11}{|l|}{ Middle East } \\
\hline Iran & KEEK & 5610 & 3420 & 2630 & 2875 & 3455 & 3150 & 2375 & 2210 & 2270 \\
\hline Iraq & KEEL & 7475 & 6680 & 14570 & 4015 & 1695 & 1415 & 945 & 1825 & 1850 \\
\hline Syria & GH5V & 140 & 110 & 70 & 110 & 350 & 330 & 160 & 155 & 155 \\
\hline Other Middle East & ZAEX & 930 & 810 & 725 & 735 & 730 & 595 & 660 & 755 & 620 \\
\hline Total Middle East & KEGY & 14150 & 11020 & 17990 & 7740 & 6225 & 5490 & 4140 & 4940 & 4895 \\
\hline \multicolumn{11}{|l|}{ Asia \& Oceania } \\
\hline Afghanistan & DMLY & 5555 & 8920 & 7205 & 2280 & 1395 & 1580 & 2400 & 2500 & 3505 \\
\hline Bangladesh & ZAEY & 795 & 510 & 720 & 735 & 510 & 425 & 440 & 540 & 455 \\
\hline China (exc Taiwan) & $\mathrm{KEGZ}$ & 4000 & 2390 & 3675 & 3450 & 2365 & 1730 & 1945 & 2100 & 1400 \\
\hline India & KEIL & 2120 & 1850 & 1865 & 2290 & 1405 & 940 & 680 & 510 & 715 \\
\hline Pakistan & KEIM & 3165 & 2860 & 2405 & 1915 & 1710 & 1145 & 965 & 1030 & 1230 \\
\hline Sri Lanka & KEIN & 6395 & 5510 & 3130 & 705 & 330 & 395 & 525 & 990 & 1475 \\
\hline Vietnam & VQIB & 180 & 400 & 840 & 1125 & 755 & 380 & 90 & 165 & 230 \\
\hline Other Asia \& Oceania & PTEE & 1025 & 1040 & 910 & 650 & 375 & 320 & 275 & 740 & 535 \\
\hline Total Asia \& Oceania & KEJO & 23230 & 23480 & 20755 & 13150 & 8850 & 6915 & 7315 & 8570 & 9550 \\
\hline Other and Nationality not known & KEJP & 450 & 160 & 150 & 55 & 70 & 105 & 55 & 75 & 75 \\
\hline Grand Total & KEJQ & 80315 & 71025 & 84130 & 49405 & 33960 & 25710 & 23610 & 23430 & 25930 \\
\hline
\end{tabular}

1 Figures rounded to the nearest 5 ( $-=0,1$ or $2, . .=$ not available/ applicable).

2 May exclude some cases lodged at Local Enforcement Offices between

January 1999 and March 2000.

3 Serbia (Inc Kosovo) and Montenegro counted separately under 'Other Europe' from 2007.

4 EU Accession States: Bulgaria, Cyprus, Czech Republic, Estonia, Hungary,

Latvia, Lithuania, Malta, Poland, Romania, Slovakia and Slovenia. Figures

between 1998 and 2000 exclude Malta but include Cyprus (Northerm part

of).

5 Provisional figures. 


\begin{tabular}{|c|c|c|c|c|c|c|c|c|c|c|c|c|}
\hline & & 1997 & 1998 & 1999 & 2000 & 2001 & 2002 & 2003 & 2004 & 2005 & 2006 & 200 \\
\hline \multicolumn{13}{|l|}{ United Kingdom } \\
\hline Marriages & KKAA & 310218 & 304797 & 301083 & 305912 & 286129 & 293021 & 308623 & 313551 & 286826 & 277611 & 27392 \\
\hline $\begin{array}{l}\text { Persons marrying per } \\
1,000 \text { resident } \\
\text { population }\end{array}$ & KKAB & 10.6 & 10.4 & 10.3 & 10.4 & 9.7 & 9.9 & 10.4 & 10.5 & 9.5 & 9.2 & \\
\hline Single men ${ }^{1}$ & KKAC & 216237 & 214005 & 211820 & 213777 & 202690 & 206196 & 217534 & 221477 & 201791 & 197137 & 196800 \\
\hline Divorced men & KKAD & 85625 & 82977 & 81750 & 84771 & 76852 & 80040 & 84011 & 85210 & 78537 & 74262 & 71101 \\
\hline Widowers & KKAE & 8356 & 7815 & 7513 & 7364 & 6587 & 6785 & 7078 & 6864 & 6498 & 6212 & 601 \\
\hline Single women ${ }^{1}$ & KKAF & 216776 & 215399 & 213246 & 215865 & 205048 & 208385 & 219828 & 224344 & 205569 & 201384 & 200518 \\
\hline Divorced women & KKAG & 85648 & 82016 & 80816 & 83166 & 74807 & 78182 & 82181 & 82559 & 75120 & 70256 & 67676 \\
\hline $\begin{array}{l}\text { Widows } \\
\text { First marriage for }\end{array}$ & $\mathrm{KKAH}$ & 7794 & 7382 & 7021 & 6881 & 6274 & 6454 & 6614 & 6648 & 6137 & 5971 & 572 \\
\hline $\begin{array}{c}\text { both partners } \\
\text { First marriage for }\end{array}$ & KMGH & 181135 & 180404 & 178759 & 180020 & 171912 & 174374 & 184661 & 188517 & 173123 & 170410 & 171 \\
\hline partners & KMGJ & 58340 & 55797 & 54776 & 56290 & 50303 & 52814 & 55922 & 56247 & 52589 & 49500 & 4768 \\
\hline \multicolumn{13}{|l|}{ Males } \\
\hline Under 21 years & KKAI & 5126 & 5173 & 5234 & 5019 & 4625 & 4396 & 4340 & 4233 & 3262 & 2821 & 261 \\
\hline $21-24$ & KKAJ & 36875 & 32723 & 29390 & 28467 & 25840 & 26293 & 27155 & 27223 & 22355 & 20768 & 20057 \\
\hline $25-29$ & KKAK & 97345 & 94696 & 90412 & 85870 & 78687 & 74858 & 75580 & 74873 & 67943 & 66234 & 66979 \\
\hline $30-34$ & KKAL & 70904 & 71096 & 72129 & 73809 & 70657 & 72592 & 75892 & 75705 & 68453 & 64688 & $622 \varepsilon$ \\
\hline $35-44$ & KKAM & 58292 & 59838 & 62114 & 68019 & 65242 & 69747 & 75695 & 79510 & 74343 & 72416 & 711 \\
\hline $45-54$ & KKAN & 26472 & 26118 & 26581 & 28791 & 26122 & 27801 & 30387 & 31851 & 30737 & 30800 & 3075 \\
\hline 55 and over & KKAO & 15204 & 15153 & 15223 & 15937 & 14956 & 17334 & 19574 & 20156 & 19733 & 19884 & 2003 \\
\hline \multicolumn{13}{|l|}{ Females } \\
\hline Under 21 years & KKAP & 17254 & 16793 & 16082 & 15938 & 13874 & 13194 & 13510 & 12878 & 9113 & 8110 & 745 \\
\hline $21-24$ & KKAQ & 59549 & 54645 & 50350 & 48578 & 45687 & 45789 & 47400 & 46891 & 39482 & 36955 & 3575 \\
\hline $25-29$ & KKAR & 97932 & 97181 & 94703 & 92753 & 85647 & 82892 & 84066 & 84714 & 79579 & 78478 & 79731 \\
\hline $30-34$ & KKAS & 58589 & 59349 & 60446 & 62478 & 59859 & 62279 & 65979 & 66508 & 61047 & 57899 & 55793 \\
\hline $35-44$ & KKAT & 47267 & 47721 & 50136 & 54697 & 52209 & 56997 & 61682 & 65007 & 60969 & 59248 & 57930 \\
\hline $45-54$ & KKAU & 21038 & 20708 & 20822 & 22621 & 20459 & 22187 & 24721 & 25846 & 24982 & 25041 & 25349 \\
\hline 55 and over & KKAV & 8589 & 8400 & 8544 & 8847 & 8394 & 9683 & 11265 & 11707 & 11654 & 11880 & 11912 \\
\hline
\end{tabular}

England and Wales

Marriages

KKBA

Persons marrying per

1,000 resident

population

KKBB

Previous marital status Single men ${ }^{1}$

Divorced men

Widowers

Single women ${ }^{1}$

Divorced women

Widows

First marriage for both partners

First marriage for

one partner

Remarriage for both partners

Males

21-24

25-29

30-34

$35-44$

$45-54$

55 and over

Females

Under 21 years

21-24

25-29

$30-34$

$35-44$

$45-54$

55 and over

KKBC

KKBD

KKBE

KKBF

KKBG

KMGK

KMGL

KMGM

KKBI

KKBP

KKB

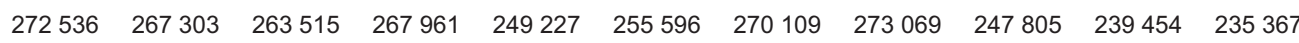

$\mathrm{KKBH}$

$\mathrm{KKBJ}$

$\mathrm{KKBK}$

KKBN

KKBO

KKBQ

KKBR

KKBU

$\mathrm{KKBV}$
$10.6 \quad 10.3$

$188268 \quad 186329 \quad 184266$

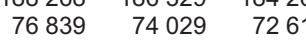

7429

$\begin{array}{rrr}188457 & 187391 & 185328 \\ 77098 & 73330 & 71971\end{array}$

6981

156907

62911

52718

5012

4574

31907

84644

62265

51654

23688

13804

4608

28389

82135

52812

23385

13651

$15439 \quad 15065$

$85352 \quad 84399$

$51405 \quad 51982$

$41838 \quad 42245$

18938

7798
$42245 \quad 44199$ \begin{tabular}{rr}
18575 & 18572 \\
7591 & 7703 \\
\hline
\end{tabular}
$51766 \quad 47446$
4629
4536 4160

25424

78364

54528

23676

43691

82250

199

67934

$14421 \quad 12467$

$42265 \quad 39746$

80312

54649

4567

20083
7986
$13682 \quad 14379$
$3952 \quad 3885$

$22961 \quad 23802$
61409

56872

22949

13467

39746
73199

51865

64619

62998

61196

24336
15534

18071

7607
$11916 \quad 12270$

$39968 \quad 41567$

$71540 \quad 72790$

$53970 \quad 57348$

$49984 \quad 54103$

$\begin{array}{rr}19535 & 21858 \\ 8683 & 10173\end{array}$ 
Population and vital statistics

52 Marriages: by previous marital status, sex, age and country

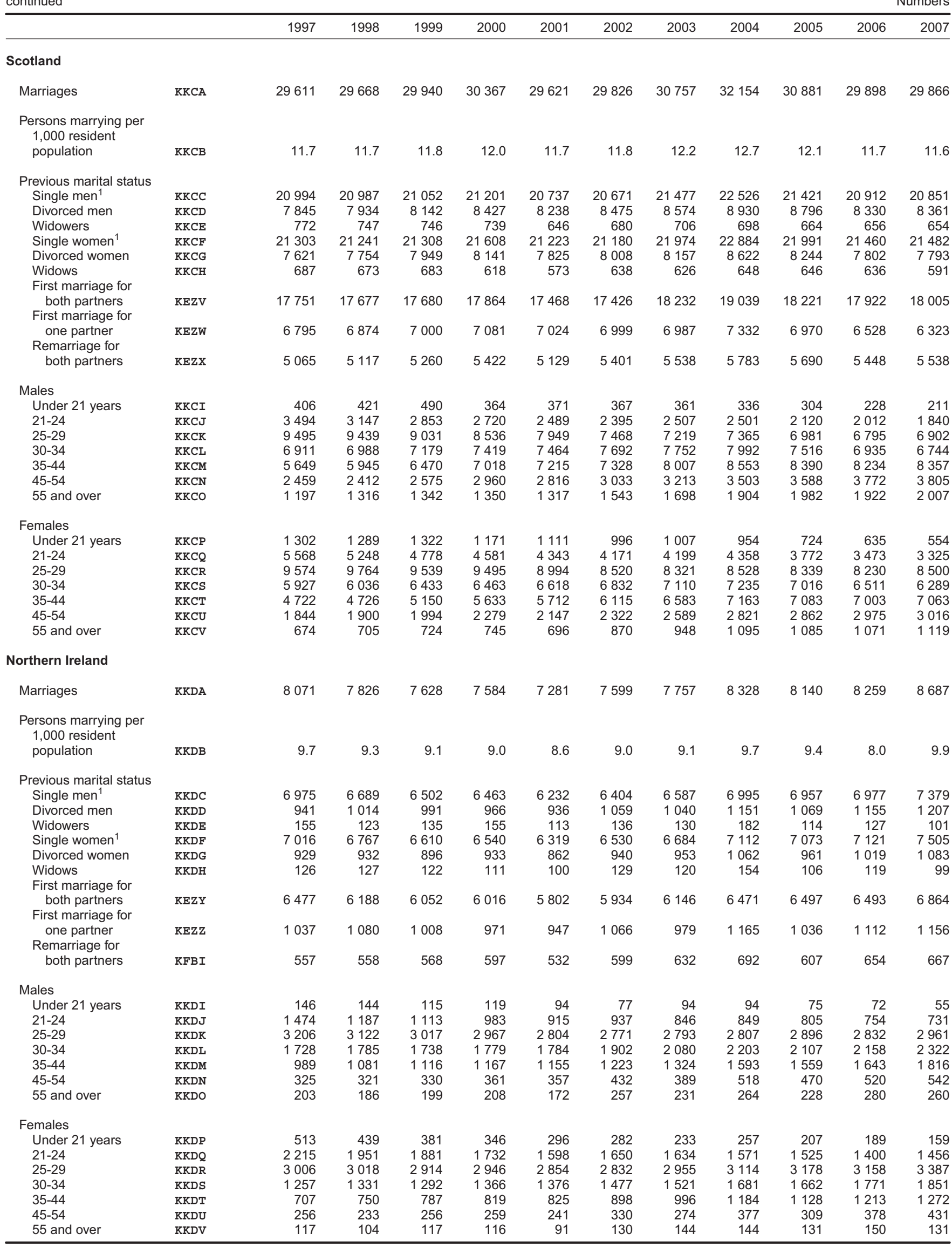

1 Single men and single women are those who have never been married.

Sources: Office for National Statistics: 01329444110 General Register Office for Scotland; Northern Ireland Statistics and Research Agency 


\section{13 Divorce: by duration of marriage, age of wife and country}

Numbers

$\begin{array}{lllllllllll}1997 & 1998 & 1999 & 2000 & 2001 & 2002 & 2003 & 2004 & 2005 & 2006 & 2007\end{array}$

\section{United Kingdom}

Decrees absolute granted ${ }^{1,2,5}$ Number

ZBRL

Duration of marriage

$0-4$ years

$5-9$ years

10-14 years

15-19 years

20 years and over

Not stated

Age of wife at marriage

$16-19$ years

20-24 years

25-29 years

30-34 years

35-39 years

40-44 years

45 years and over

Not stated

Age of wife at divorce

16-24 years

25-29 years

30-34 years

35-39 years

40-44 years

45 years and over

Not stated

Divorces in which there were ${ }^{3,4}$

No children aged under 16

One or more children aged under 16

\section{England and Wales}

Decrees absolute granted ${ }^{1,2}$

Number

KKEA

Rate per 1,000 married couples

KKEB

Duration of marriage

$0-4$ years

5-9 years

10-14 years

$15-19$ years

20 years and over

Not stated

Age of wife at marriage

16-19 years

20-24 years

25-29 years

30-34 years

35-39 years

40-44 years

45 years and over

Age of wife at divorce

16-24 years

25-29 years

30-34 years

35-39 years

40-44 years

45 years and over

Not stated

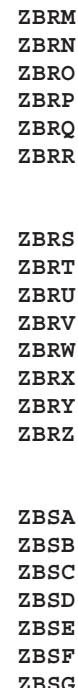

BRN

BRO

BRP

BRR

BRS

ZBRU

ZBRV

BRX

ZBRZ

BSA

BSB

BSD

BSE

ZBSG

ZBSF
997

1998

2000

2007

Divorces in which there were ${ }^{3}$

No children aged under 16

One or more children aged under 16 zBSK
$161087160057 \quad 158746 \quad 154628 \quad 156814 \quad 160726 \quad 166737 \quad 167138 \quad 155052 \quad 148141 \quad 144220$

$\begin{array}{llllll}33719 & 33087 & 31047 & 28933 & 28306 & 28591 \\ 45040 & 44243 & 43357 & 41621 & 42360 & 42924\end{array}$

$29085 \quad 29706 \quad 30270 \quad 30166$

$20211 \quad 20078 \quad 20147 \quad 19902$

$42360 \quad 42924$

33020

33916

34000
6

28781

43558
32564

32564
23119

28746

42855
31775

26549

$25005 \quad 24704$

34729
2

$\begin{array}{rr}38713 & 39844 \\ 2 & 20\end{array}$

$21007-27647-26446$

$21244 \quad 20894$

$37116 \quad 36397$

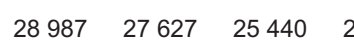

$\begin{array}{llll}72971 & 71416 & 69509 & 66215\end{array}$

33452

$34195 \quad 35585 \quad 36009$

22558

22107

22367

20948

18507

$\begin{array}{llll}12968 & 13719 & 14420 & 14892\end{array}$

$37418 \quad 39264$

$67070 \quad 65671$

58829

$15842 \quad 17374$

41464
18658

$55085 \quad 52438$

$\begin{array}{lllll}3375 & 3360 & 3557 & 3568 & 7417\end{array}$

30943086

3557
3291

3568
3352

$3778 \quad 4104$

8742

19729

19366

$38812 \quad 38315$

$4404 \quad 4550 \quad 4703 \quad 4677 \quad 4784$

$\begin{array}{lllll}3917 & 4093 & 4042 & 3985 & 4081\end{array}$

$\begin{array}{lllllllllll}7371 & 6758 & 5671 & 5115 & 4874 & 4998 & 5092 & 4885 & 4388 & 3739 & 3492\end{array}$

$\begin{array}{lllllllllll}28814 & 26968 & 24120 & 21280 & 19635 & 18340 & 17633 & 16972 & 14870 & 14216 & 13772\end{array}$

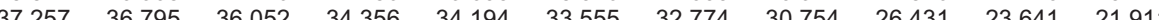

$\begin{array}{lllllllllll}30641 & 31688 & 32605 & 32588 & 33997 & 35050 & 36465 & 35894 & 32722 & 30567 & 28765\end{array}$

$\begin{array}{lllllllllll}22246 & 22810 & 23614 & 23879 & 25579 & 27564 & 30154 & 31372 & 30359 & 29412 & 28990\end{array}$

$\begin{array}{lllllllllllll}34662 & 34947 & 36578 & 37311 & 38442 & 41102 & 44498 & 47108 & 46085 & 46352 & 47071\end{array}$

$96 \quad 91 \quad 106$

311
99

$93 \quad 117$
$20568 \quad 21881$

$16707 \quad 15430$

$6155 \quad 6571 \quad 6848 \quad 6993$

4254440143

$146689145214 \quad 144556141135 \quad 143818 \quad 147735 \quad 153490 \quad 153399 \quad 141750 \quad 132562 \quad 128534$

\begin{tabular}{|c|c|c|c|c|c|c|c|c|c|c|}
\hline 13.0 & 12.9 & 12.9 & 12.7 & 12.9 & 13.4 & 14.0 & 14.1 & 13.0 & 12.2 & 12.0 \\
\hline 31767 & 31136 & 29307 & 27474 & 26987 & 27344 & 27511 & 27389 & 25345 & 23427 & 23039 \\
\hline 41260 & 40239 & 39676 & 38206 & 39079 & 39730 & 40599 & 39779 & 36161 & 33864 & 32522 \\
\hline 26215 & 26698 & 27384 & 27459 & 28176 & 28592 & 29831 & 29086 & 26394 & 24680 & 23496 \\
\hline 18027 & 17934 & 18072 & 17870 & 18603 & 19784 & 20923 & 21591 & 20363 & 18792 & 18266 \\
\hline 29408 & 29199 & 30108 & 30120 & 30971 & 32285 & 34624 & 35554 & 33478 & 31786 & 31204 \\
\hline 12 & 8 & 9 & 6 & 2 & - & 2 & - & 9 & 13 & 7 \\
\hline 25579 & 24276 & 22486 & 20930 & 20218 & 19828 & 20063 & 18709 & 16519 & 14478 & 13273 \\
\hline 66167 & 64453 & 62853 & 59874 & 60211 & 60353 & 61057 & 59548 & 53041 & 48550 & 45929 \\
\hline 31022 & 31533 & 32867 & 33282 & 34759 & 36387 & 38722 & 39575 & 37103 & 35177 & 34675 \\
\hline 12094 & 12788 & 13507 & 13972 & 14890 & 16339 & 17567 & 18545 & 18138 & 17834 & 17637 \\
\hline 5767 & 6153 & 6432 & 6562 & 6956 & 7623 & 8249 & 8912 & 8755 & 8560 & 8880 \\
\hline 3156 & 3135 & 3331 & 3378 & 3559 & 3841 & 4154 & 4274 & 4421 & 4318 & 4393 \\
\hline 2904 & 2876 & 3080 & 3137 & 3225 & 3364 & 3678 & 3836 & 3773 & 3645 & 3747 \\
\hline 6871 & 6298 & 5318 & 4839 & 4643 & 4808 & 4867 & 4658 & 4216 & 3525 & 3273 \\
\hline 26435 & 24586 & 22173 & 19650 & 18231 & 17227 & 16539 & 15867 & 13905 & 13182 & 12653 \\
\hline 33967 & 33446 & 32837 & 31420 & 31489 & 30982 & 30345 & 28368 & 24381 & 21409 & 19865 \\
\hline 27715 & 28605 & 29663 & 29820 & 31164 & 32282 & 33519 & 33013 & 29864 & 27479 & 25665 \\
\hline 20125 & 20521 & 21325 & 21469 & 23190 & 25017 & 27610 & 28558 & 27570 & 26128 & 25548 \\
\hline 31564 & 31750 & 33231 & 33931 & 35099 & 37419 & 40608 & 42935 & 41805 & 40826 & 41523 \\
\hline 12 & 8 & 9 & 6 & 2 & - & 2 & - & 9 & 13 & 7 \\
\hline 66019 & 64738 & 65258 & 64359 & 64541 & 66738 & 69681 & 71382 & 88349 & 62667 & 62497 \\
\hline 80670 & 80476 & 79298 & 76776 & 79277 & 80997 & 83809 & 82017 & 75340 & 69895 & 66037 \\
\hline
\end{tabular}




\begin{tabular}{|c|c|c|c|c|c|c|c|c|c|c|c|c|}
\hline & & 1997 & 1998 & 1999 & 2000 & 2001 & 2002 & 2003 & 2004 & 2005 & 2006 & 2007 \\
\hline \multicolumn{13}{|l|}{ Scotland } \\
\hline \multicolumn{13}{|l|}{ Decrees absolute granted ${ }^{2}$} \\
\hline Number & KKFA & 12222 & 12384 & 11864 & 11143 & 10631 & 10826 & 10928 & 11227 & 10940 & 13014 & 12773 \\
\hline Rate per 1,000 married couples & KKFB & 11.0 & 11.3 & 10.9 & 10.3 & 9.7 & 10.0 & 10.2 & 10.5 & 10.3 & 12.3 & 12.2 \\
\hline \multicolumn{13}{|l|}{ Duration of marriage } \\
\hline $0-4$ years & KKFC & 1793 & 1766 & 1588 & 1304 & 1159 & 1128 & 1141 & 1204 & 1089 & 1444 & 1526 \\
\hline $5-9$ years & KKFD & 3224 & 3360 & 3095 & 2890 & 2721 & 2689 & 2450 & 2536 & 2403 & 2759 & 2659 \\
\hline $10-14$ years & KKFE & 2385 & 2456 & 2368 & 2168 & 2163 & 2183 & 2222 & 2173 & 2113 & 2418 & 2345 \\
\hline $15-19$ years & KKFF & 1804 & 1729 & 1686 & 1622 & 1562 & 1705 & 1773 & 1810 & 1789 & 2033 & 2100 \\
\hline 20 years and over & KKFG & 3016 & 3073 & 3127 & 3159 & 3026 & 3121 & 3342 & 3504 & 3546 & 4360 & 4143 \\
\hline Not stated & ZBSL & - & - & - & - & - & - & - & - & - & - & - \\
\hline \multicolumn{13}{|l|}{ Age of wife at marriage } \\
\hline $16-19$ years & ZBSM & 2749 & 2654 & 2374 & 2043 & 1839 & 1845 & 1816 & 1753 & 1557 & 1764 & 1700 \\
\hline 20-24 years & ZBSN & 5714 & 5744 & 5453 & 5142 & 4873 & 4823 & 4869 & 4892 & 4721 & 5373 & 5141 \\
\hline $35-39$ years & KKFL & 360 & 382 & 379 & 378 & 401 & 407 & 432 & 489 & 455 & 619 & 699 \\
\hline 40-44 years & KKFM & 199 & 198 & 208 & 170 & 193 & 235 & 219 & 252 & 252 & 325 & 336 \\
\hline 45 years and over & KKFN & 173 & 185 & 192 & 193 & 175 & 186 & 212 & 221 & 232 & 312 & 298 \\
\hline Not stated & KKFO & 85 & 83 & 96 & 94 & 90 & 119 & 115 & 133 & 143 & 159 & 196 \\
\hline \multicolumn{13}{|l|}{ Age of wife at divorce } \\
\hline $16-24$ years & KKFP & 426 & 377 & 301 & 232 & 182 & 180 & 191 & 192 & 148 & 190 & 199 \\
\hline $25-29$ years & KKFQ & 2021 & 1957 & 1597 & 1330 & 1109 & 974 & 884 & 869 & 777 & 877 & 938 \\
\hline $30-34$ years & KKFR & 2736 & 2767 & 2642 & 2381 & 2215 & 2174 & 1943 & 1918 & 1641 & 1837 & 1655 \\
\hline $35-39$ years & KKFS & 2469 & 2562 & 2450 & 2298 & 2311 & 2281 & 2388 & 2278 & 2304 & 2544 & 2445 \\
\hline $40-44$ years & KKFT & 1819 & 1951 & 1929 & 1999 & 1963 & 2110 & 2106 & 2341 & 2330 & 2751 & 2801 \\
\hline 45 years and over & KKFU & 2667 & 2687 & 2848 & 2810 & 2760 & 2990 & 3297 & 3496 & 3596 & 4650 & 4542 \\
\hline Not stated & KKFV & 84 & 83 & 97 & 93 & 91 & 117 & 119 & 133 & 144 & 165 & 193 \\
\hline \multicolumn{13}{|l|}{ Divorces in which there were ${ }^{3,4}$} \\
\hline No children aged under 16 & KKFW &.. &.. &.. & .. &.. & .. &.. & .. & .. & .. & .. \\
\hline One or more children under 16 & KKFX &.. &.. &.. & .. &.. & .. &.. & .. & .. & .. & .. \\
\hline Number & zBSO & 2176 & 2459 & 2326 & 2350 & 2365 & 2165 & 2319 & 2512 & 2362 & 2565 & 2913 \\
\hline \multicolumn{13}{|l|}{ Duration of marriage } \\
\hline 0-4 years & ZBSP & 159 & 185 & 152 & 155 & 160 & 119 & 129 & 153 & 115 & 134 & 139 \\
\hline $5-9$ years & ZBSQ & 556 & 644 & 586 & 525 & 560 & 505 & 509 & 540 & 506 & 493 & 591 \\
\hline $10-14$ years & ZBSR & 485 & 552 & 518 & 539 & 510 & 482 & 511 & 516 & 500 & 549 & 605 \\
\hline $15-19$ years & ZBSS & 380 & 415 & 389 & 410 & 403 & 392 & 423 & 497 & 441 & 419 & 528 \\
\hline 20 years and over & ZBST & 596 & 663 & 681 & 721 & 732 & 667 & 747 & 786 & 800 & 970 & 1050 \\
\hline Not stated ${ }^{5}$ & EK8B & - & - & - & - & - & - & - & 20 & - & .. & .. \\
\hline \multicolumn{13}{|l|}{ Age of wife at marriage } \\
\hline $16-19$ years & ZBSU & 659 & 697 & 580 & 532 & 501 & 434 & 488 & 486 & 431 & 465 & 457 \\
\hline 20-24 years & ZBSV & 1090 & 1219 & 1203 & 1199 & 1198 & 1088 & 1144 & 1231 & 1067 & 1162 & 1368 \\
\hline $25-29$ years & zBSW & 279 & 348 & 385 & 409 & 426 & 413 & 435 & 507 & 525 & 560 & 641 \\
\hline $30-34$ years & zBSX & 83 & 107 & 84 & 115 & 125 & 140 & 133 & 159 & 163 & 198 & 253 \\
\hline $35-39$ years & ZBSY & 28 & 36 & 37 & 53 & 60 & 40 & 61 & 55 & 65 & 82 & 84 \\
\hline $40-44$ years & ZBSZ & 20 & 27 & 18 & 20 & 26 & 28 & 31 & 24 & 30 & 34 & 55 \\
\hline 45 years and over & ZBTA & 17 & 25 & 19 & 22 & 29 & 22 & 27 & 36 & 37 & 28 & 36 \\
\hline Not stated ${ }^{6}$ & EK8C & - & - & - & - & - & - & - & 14 & 44 & 36 & 19 \\
\hline \multicolumn{13}{|l|}{ Age of wife at divorce } \\
\hline $16-24$ years & ZВTB & 74 & 83 & 52 & 44 & 49 & 10 & 34 & 35 & 24 & 24 & 20 \\
\hline $25-29$ years & ZBTC & 358 & 425 & 350 & 300 & 295 & 139 & 210 & 236 & 188 & 157 & 181 \\
\hline $30-34$ years & ZBTD & 554 & 582 & 573 & 555 & 490 & 399 & 486 & 468 & 409 & 395 & 391 \\
\hline $35-39$ years & ZBTE & 457 & 521 & 492 & 470 & 522 & 487 & 558 & 603 & 554 & 544 & 655 \\
\hline 40-44 years & ZBTF & 302 & 338 & 360 & 411 & 426 & 437 & 438 & 473 & 459 & 533 & 641 \\
\hline 45 years and over & ZBTG & 431 & 510 & 499 & 570 & 583 & 693 & 593 & 677 & 684 & 876 & 1006 \\
\hline Not stated ${ }^{6}$ & EK8D & - & - & - & - & - & - & - & 20 & 44 & 36 & 19 \\
\hline Divorces in which there were ${ }^{3}$ & & & & & & & & & & & & \\
\hline No children aged under 16 & $\mathrm{ZBTH}$ & 1573 & 1807 & 1649 & 1051 & 1054 & 972 & 1050 & 1218 & 982 & 662 & 1372 \\
\hline One or more children aged under 16 & ZBTI & 603 & 652 & 677 & 1299 & 1311 & 1193 & 1269 & 1282 & 1380 & 1903 & 1541 \\
\hline Not stated ${ }^{6}$ & EK8E & - & - & - & - & - & - & - & 12 & - &.. & (1) \\
\hline
\end{tabular}

1 Data for 2007 are provisional.

Sources: Office for National Statistics: 01329444410

2 Includes decrees of nullities.

General Register Office for Scotland;

3 Children of the family as defined by the Matrimonial Causes Act 1973. Northern Ireland Statistics and Research Agency

4 Data not available in Scotland.

5 Marital estimates are not available for Northern Ireland - no divorce rate for

UK/Northern Ireland.

6 Due to some incomplete records. 


$\begin{array}{lllllllllll}1997 & 1998 & 1999 & 2000 & 2001 & 2002 & 2003 & 2004 & 2005 & 2006 & 2007\end{array}$

\section{United Kingdom}

Dissolution of marriage $e^{1,4}$

Decree absolute/decree granted ZBXR

On grounds of:

Adultery

Behaviour

Desertion

Separation (2 years and

consent)

Separation( 5 years)

Combination of more than one ground and other Separation $^{2}$

1 year and consent

2 years

ZBXS
ZBXT
ZBXU
ZBXV
ZBXW
ZBXX
IE9T
IE9U

Decree absolute/decree granted to: the wife

the husband

ZBXY

both

ZBXZ

ZBYA

Nullity of marriage

Decree absolute/decree granted ZBYB

England and Wales

\begin{tabular}{|c|}
\hline $\begin{array}{l}\text { Dissolution of marriage }{ }^{4} \\
\text { Petitions filed } \\
\text { Decree nisi granted }^{3} \\
\text { Decree absolute granted }\end{array}$ \\
\hline $\begin{array}{l}\text { On grounds of: } \\
\text { Adultery } \\
\text { Behaviour } \\
\text { Desertion } \\
\text { Separation (2 years and } \\
\text { consent) } \\
\text { Separation(5 years) } \\
\text { Combination of more than } \\
\text { one ground and other }\end{array}$ \\
\hline $\begin{array}{l}\text { Decree absolute granted to: } \\
\text { the wife } \\
\text { the husband } \\
\text { both }\end{array}$ \\
\hline $\begin{array}{l}\text { Nullity of marriage } \\
\text { Petitions filed }{ }^{3} \\
\text { Decree nisi granted } \\
\text { Decree absolute granted }\end{array}$ \\
\hline $\begin{array}{l}\text { Judicial separation } \\
\text { Petitions filed } \\
\text { Decrees granted }^{4}\end{array}$ \\
\hline
\end{tabular}

$160733159688 \quad 158418 \quad 154273 \quad 156562 \quad 160528 \quad 166536 \quad 166937 \quad 154879 \quad 147989 \quad 144071$

$$
\begin{aligned}
& \begin{array}{lllllllllll}
38652 & 37302 & 35545 & 34082 & 33452 & 33389 & 33844 & 32586 & 28411 & 25293 & 23355
\end{array} \\
& \begin{array}{lllllllllll}
68546 & 68685 & 67851 & 65687 & 66818 & 68499 & 70866 & 70879 & 66824 & 63782 & 62238
\end{array}
\end{aligned}
$$

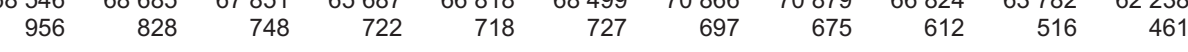

$$
\begin{aligned}
& \begin{array}{lllllllllll}
39398 & 39627 & 40368 & 39763 & 40699 & 42579 & 44012 & 44819 & 41433 & 36917 & 33612
\end{array} \\
& \begin{array}{lllllllllll}
12552 & 12697 & 13389 & 13653 & 14575 & 15076 & 16831 & 17714 & 17101 & 15297 & 13192
\end{array} \\
& \begin{array}{lllllllllll}
629 & 549 & 517 & 366 & 300 & 258 & 286 & 264 & 498 & 601 & 325
\end{array} \\
& \begin{array}{llllllllllllll}
. . & . . & . . & . . & . . & . & . & . & . & & . . & 1456 & 3003
\end{array} \\
& \begin{array}{llllllllllll}
111910 & 111555 & 109824 & 106957 & 107345 & 108104 & 114664 & 113970 & 105008 & 100003 & 96749
\end{array} \\
& \begin{array}{lllllllllll}
48393 & 47764 & 48236 & 47069 & 49015 & 52251 & 51691 & 52793 & 49725 & 47847 & 47205
\end{array}
\end{aligned}
$$

\begin{tabular}{|c|c|c|c|c|c|c|c|c|c|c|c|}
\hline KKGA & 163769 & 165870 & 162137 & 157809 & 172341 & 177224 & 173265 & 167340 & 151824 & 148564 & 137465 \\
\hline KKGM & 148310 & 144231 & 143446 & 143729 & 163146 & 170980 & 168037 & 166334 & 150917 & 145242 & 143153 \\
\hline KKGN & 146339 & 144851 & 144233 & 140783 & 143568 & 147538 & 153294 & 153199 & 141583 & 132418 & 128393 \\
\hline KKGB & 37592 & 36319 & 34584 & 33310 & 32839 & 32829 & 33331 & 32035 & 27992 & 24936 & 23125 \\
\hline KKGC & 65047 & 65257 & 64816 & 63182 & 64768 & 66480 & 68944 & 68859 & 65169 & 62234 & 61004 \\
\hline KKGD & 912 & 790 & 713 & 680 & 689 & 681 & 665 & 654 & 593 & 499 & 451 \\
\hline KKGE & 32638 & 32394 & 33482 & 32820 & 33703 & 35476 & 36931 & 37543 & 34388 & 31794 & 31268 \\
\hline KKGF & 9592 & 9616 & 10193 & 10498 & 11355 & 11896 & 13239 & 13933 & 13196 & 12628 & 12220 \\
\hline ZBYC & 558 & 475 & 445 & 293 & 214 & 176 & 184 & 175 & 245 & 327 & 325 \\
\hline ZBYD & 102173 & 101583 & 100469 & 98227 & 98992 & 102676 & 106208 & 105381 & 96855 & 90587 & 87362 \\
\hline ZBYE & 43739 & 42902 & 43413 & 42311 & 44378 & 44694 & 46915 & 47651 & 44583 & 41702 & 40928 \\
\hline ZBYF & 427 & 366 & 351 & 245 & 198 & 168 & 171 & 167 & 145 & 129 & 103 \\
\hline KKGO & 485 & 505 & 549 & 452 & 492 & 443 & 463 & 495 & 440 & 406 & 352 \\
\hline KKGR & 248 & 281 & 495 & 274 & 160 & 216 & 204 & 308 & 260 & 240 & 190 \\
\hline KKGS & 350 & 363 & 323 & 352 & 250 & 197 & 196 & 200 & 167 & 144 & 141 \\
\hline KKGT & 1078 & 916 & 882 & 650 & 1078 & 1001 & 826 & 745 & 697 & 613 & 50 \\
\hline KKGW & 589 & 519 & 696 & 540 & 925 & 560 & 467 & 419 & 387 & 353 & 32 \\
\hline
\end{tabular}

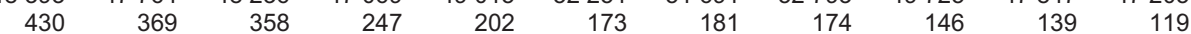

$$
\begin{aligned}
& \begin{array}{lllllllllll}
354 & 369 & 328 & 355 & 252 & 198 & 201 & 201 & 173 & 152 & 149
\end{array}
\end{aligned}
$$


Population and vital statistics

4 Divorce proceedings: by country

$1997 \quad 1998 \quad 1999$

2000

$2001 \quad 2002$

2003

2004

2005

Scotland

Dissolution of marriage ${ }^{1}$ Decree granted

On grounds of:

Adultery

Behaviour

Desertion

Separation (2 years and consent)

Separation (5 years) Separation ${ }^{2}$

1 year and consent

2 years

\begin{tabular}{|c|c|c|c|c|c|c|c|c|c|c|c|}
\hline ZBYG & 12220 & 12383 & 11860 & 11142 & 10631 & 10825 & 10927 & 11226 & 10939 & 13013 & 12771 \\
\hline ZBYH & 909 & 832 & 770 & 610 & 473 & 428 & 401 & 413 & 327 & 263 & 131 \\
\hline ZBYI & 3081 & 3005 & 2611 & 2099 & 1639 & 1656 & 1537 & 1546 & 1344 & 1215 & 875 \\
\hline ZBYJ & 33 & 28 & 18 & 34 & 24 & 42 & 23 & 15 & 17 & 15 & 7 \\
\hline ZBYK & 5773 & 6121 & 5908 & 5878 & 5943 & 6101 & 6016 & 6122 & 5989 & 4014 & 971 \\
\hline ZBYL & 2424 & 2397 & 2553 & 2521 & 2552 & 2598 & 2950 & 3130 & 3262 & 1923 & 182 \\
\hline IE9T & .. & .. & .. & .. & .. & .. & .. & .. & .. & 1456 & 3003 \\
\hline IE9U & .. & .. & .. & .. & .. & .. & .. & .. & .. & 4127 & 4127 \\
\hline ZBYM & 8266 & 8328 & 7770 & 7190 & 6775 & 6800 & 6926 & 6938 & 6653 & 7750 & 7528 \\
\hline ZBYN & 3954 & 4055 & 4090 & 3952 & 3856 & 4025 & 4001 & 4288 & 4286 & 5263 & 5245 \\
\hline
\end{tabular}

Decree granted to ${ }^{2}$

the wife

the husband

$3952 \quad 385$

$4025 \quad 4001$

\section{Nullity of marriage \\ Decree granted}

ZBYO

2

Northern Ireland

Dissolution of marriage

Petitions filed

Decree nisi granted

Decree absolute granted

ZBYP

ZBYQ

$2808 \quad 2760 \quad 2414$

$\begin{array}{lll}2532 & 2904 & 2393 \\ 2174 & 2454 & 2325\end{array}$

3005

2456

2869

2615

2929

2454
2165

3192

2616
2315

2808
2697

2594

3098

2607

On grounds of:

Adultery

Behaviour

Separation (2 years and

consent)

Separation(5 years)

Combination of more than

one ground and other

ZBYS

$151 \quad 151 \quad 191$

$162 \quad 140$

$\begin{array}{llll}2512 & 2357 \quad 2558 & 2907\end{array}$

ZBYT

$\begin{array}{rrr}418 & 423 & 424 \\ 11 & 10 & 17\end{array}$

406

ZBYV

$991 \quad 1112$

978

1065

140
411
5

112

$\begin{array}{llrr}138 & 92 & 94 & 99\end{array}$

ZBYW

$536 \quad 684$

1053

ZBYX

67

634

668

$\begin{array}{llll}1154 & 1056 \quad 1109 & 1373\end{array}$

Decree absolute granted to:

the wife

the husband

ZBYY

ZBYZ

ZBZA

$1473 \quad 1644$

$73 \quad 86$

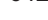

643

274

283

both

ZBZB

Petitions filed

Decree nisi granted

Decree absolute granted

ZBZC

ZBZD

Judicial separation

Petitions filed

$\begin{array}{lll}\text { ZBZE } & 70 & 64\end{array}$

$1540 \quad 1578$

$578 \quad 1405$

153

$1651 \quad 1500 \quad 1666 \quad 1859$

$\begin{array}{lr}3 & 3\end{array}$

2

$\begin{array}{rr}755 & 775 \\ 5 & 10\end{array}$

854

1

8821032

Decrees granted

ZBZF

34

40

31

25

1
2
2

1 The terms petition filed, decree nisi granted, decree absolute and judicial separation are not used in Scotland. Decree absolute granted to 'both' and 'Combination of more than one ground and other' are not procedures used in Scotland.

2 New categories introduced with effect from 4 May 2006 by the Family Law (Scotland) Act 2006. These replace the two 'non-cohabitation' categories (non-cohabiation is a category that is used in Scotland only) of 2 years with consent and 5 years.

3 Data supplied by Ministry of Justice (12 February 2008) see Judicial and

Court Statistics.

42007 data are provisional. 


\begin{tabular}{|c|c|c|c|c|c|c|c|c|c|}
\hline & \multicolumn{3}{|c|}{ Live births } & \multirow[b]{2}{*}{$\begin{array}{l}\text { Sex } \\
\text { ratio }^{2}\end{array}$} & \multicolumn{2}{|c|}{ Rates } & \multirow[b]{2}{*}{$\mathrm{TFR}^{5}$} & \multirow[b]{2}{*}{$\begin{array}{l}\text { Still- } \\
\text { births }\end{array}$} & \multirow[b]{2}{*}{$\begin{array}{l}\text { Still- } \\
\text { birth } \\
\text { rate }^{6}\end{array}$} \\
\hline & Total & Male & Female & & $\begin{array}{r}\text { Crude } \\
\text { birth } \\
\text { rate }^{3}\end{array}$ & $\begin{array}{c}\text { General } \\
\text { fertility } \\
\text { rate }^{4}\end{array}$ & & & \\
\hline \multicolumn{10}{|c|}{ United Kingdom $^{7}$} \\
\hline $1900-02$ & 1095 & 558 & 537 & 1037 & 28.6 & 115.1 &.. & .. & .. \\
\hline $1910-12$ & 1037 & 528 & 508 & 1039 & 24.6 & 99.4 &.. & .. & .. \\
\hline $1920-22$ & 1018 & 522 & 496 & 1052 & 23.1 & 93.0 &.. & .. & .. \\
\hline $1930-32$ & 750 & 383 & 367 & 1046 & 16.3 & 66.5 & & .. & .. \\
\hline $1940-42$ & 723 & 372 & 351 & 1062 & 15.0 & & 1.89 & .. & .. \\
\hline $1950-52$ & 803 & 413 & 390 & 1061 & 16.0 & 73.7 & 2.21 & .. & .. \\
\hline $1960-62$ & 946 & 487 & 459 & 1063 & 17.9 & 90.3 & 2.80 & 18.6 & 19.2 \\
\hline $1970-72$ & 880 & 453 & 427 & 1064 & 15.8 & 82.5 & 2.36 & 11.3 & 12.7 \\
\hline $1980-82$ & 735 & 377 & 358 & 1053 & 13.0 & 62.5 & 1.83 & 5.0 & 6.8 \\
\hline $1990-92$ & 790 & 405 & 385 & 1051 & 13.8 & 63.7 & 1.81 & 3.6 & 4.6 \\
\hline \multirow[t]{2}{*}{$2000-02$} & 672 & 345 & 328 & 1052 & 11.4 & 54.7 & 1.64 & 3.6 & 5.4 \\
\hline & BBCA & $\mathrm{KBCZ}$ & $\mathrm{KBCY}$ & KMFW & КBCT & KBCS & KBCR & $\mathrm{KBCQ}$ & KMFX \\
\hline 1998 & 716.9 & 367 & 350 & 1052 & 12.3 & 58.8 & 1.71 & 3.9 & 5.4 \\
\hline 1999 & 700.0 & 359 & 341 & 1056 & 11.9 & 57.3 & 1.68 & 3.7 & 5.3 \\
\hline 2000 & 679.0 & 348 & 331 & 1051 & 11.5 & 55.4 & 1.64 & 3.6 & 5.3 \\
\hline 2001 & 669.1 & 343 & 326 & 1050 & 11.3 & 54.3 & 1.63 & 3.6 & 5.3 \\
\hline 2002 & 668.8 & 343 & 326 & 1054 & 11.3 & 54.2 & 1.64 & 3.8 & 5.6 \\
\hline 2003 & 695.6 & 357 & 339 & 1052 & 11.7 & 56.2 & 1.71 & 4.0 & 5.7 \\
\hline 2004 & 716.0 & 368 & 348 & 1055 & 12.0 & 57.7 & 1.77 & 4.0 & 5.7 \\
\hline 2005 & 722.5 & 370 & 353 & 1050 & 12.0 & 57.8 & 1.78 & 4.0 & 5.3 \\
\hline 2006 & 748.6 & 383 & 366 & 1047 & 12.4 & 59.7 & 1.84 & 4.0 & 5.3 \\
\hline 2007 & 772.2 & 397 & 376 & 1056 & 12.7 & 61.5 & 1.90 & 4.0 & 5.2 \\
\hline 2008 & 794.4 & 407 & 388 & 1049 & 12.9 & 63.4 & 1.96 & 4.0 & 5.1 \\
\hline \multicolumn{10}{|c|}{ England and Wales } \\
\hline $1900-02$ & 932 & 475 & 458 & 1037 & 28.6 & 114.7 & .. & .. & .. \\
\hline $1910-12$ & 884 & 450 & 433 & 1040 & 24.5 & 98.6 &.. & .. & .. \\
\hline $1920-22$ & 862 & 442 & 420 & 1051 & 22.8 & 91.1 & .. & .. & .. \\
\hline $1930-32$ & 632 & 323 & 309 & 1047 & 15.8 & 64.4 & .. & 27.0 & .. \\
\hline $1940-42$ & 607 & 312 & 295 & 1057 & 15.6 & 61.3 & 1.81 & 22.0 & .. \\
\hline $1950-52$ & 683 & 351 & 332 & 1058 & 15.6 & 72.1 & 2.16 & 16.0 & .. \\
\hline $1960-62$ & 812 & 418 & 394 & 1061 & 17.6 & 88.9 & 2.77 & 15.6 & 18.9 \\
\hline $1970-72$ & 764 & 394 & 371 & 1061 & 15.6 & 81.4 & 2.31 & 9.7 & 12.5 \\
\hline $1980-82$ & 639 & 328 & 311 & 1053 & 12.9 & 61.8 & 1.81 & 4.3 & 6.7 \\
\hline $1990-92$ & 698 & 358 & 340 & 1051 & 13.8 & 63.8 & 1.82 & 3.2 & 4.5 \\
\hline \multirow[t]{2}{*}{$2000-02$} & 598 & 307 & 292 & 1052 & 11.4 & 55.2 & 1.65 & 3.2 & 5.4 \\
\hline & $\mathrm{BBCB}$ & KMFY & KMFZ & KMGA & KMGB & KMGC & KMGD & KMGE & KMGF \\
\hline 1998 & 635.9 & 326 & 310 & 1051 & 12.3 & 59.2 & 1.72 & 3.4 & 5.3 \\
\hline 1999 & 621.9 & 319 & 303 & 1055 & 12.0 & 57.8 & 1.70 & 3.3 & 5.3 \\
\hline 2000 & 604.4 & 310 & 295 & 1050 & 11.6 & 55.9 & 1.65 & 3.2 & 5.3 \\
\hline 2001 & 594.6 & 305 & 290 & 1050 & 11.4 & 54.7 & 1.63 & 3.2 & 5.3 \\
\hline 2002 & 596.1 & 306 & 290 & 1055 & 11.3 & 54.7 & 1.65 & 3.4 & 5.6 \\
\hline 2003 & 621.5 & 318 & 303 & 1051 & 11.8 & 56.8 & 1.73 & 3.6 & 5.8 \\
\hline 2004 & 639.7 & 328 & 311 & 1054 & 12.1 & 58.2 & 1.78 & 3.7 & 5.7 \\
\hline 2005 & 645.8 & 331 & 315 & 1049 & 12.1 & 58.4 & 1.80 & 3.5 & 5.4 \\
\hline 2006 & 669.6 & 342 & 327 & 1047 & 12.5 & 60.2 & 1.86 & 3.6 & 5.4 \\
\hline 2007 & 690.0 & 354 & 336 & 1057 & 12.8 & 62.0 & 1.92 & 3.6 & 5.2 \\
\hline 2008 & 708.7 & 363 & 346 & 1050 & 13.0 & 63.8 & 1.97 & 3.6 & 5.1 \\
\hline
\end{tabular}




\begin{tabular}{|c|c|c|c|c|c|c|c|c|c|}
\hline & \multicolumn{3}{|c|}{ Live births } & \multirow[b]{2}{*}{$\begin{array}{l}\text { Sex } \\
\text { ratio }^{2}\end{array}$} & \multicolumn{2}{|c|}{ Rates } & \multirow[b]{2}{*}{ TFR $^{5}$} & \multirow[b]{2}{*}{$\begin{array}{l}\text { Still- } \\
\text { births }^{6}\end{array}$} & \multirow[b]{2}{*}{$\begin{array}{l}\text { Still- } \\
\text { birth } \\
\text { rate }^{6}\end{array}$} \\
\hline & Total & Male & Female & & $\begin{array}{r}\text { Crude } \\
\text { birth } \\
\text { rate }^{3}\end{array}$ & $\begin{array}{c}\text { General } \\
\text { fertility } \\
\text { rate }^{4}\end{array}$ & & & \\
\hline \multicolumn{10}{|l|}{ Scotland } \\
\hline $1900-02$ & 132 & 67 & 65 & 1046 & 29.5 & 120.6 & .. & .. & .. \\
\hline $1910-12$ & 123 & 63 & 60 & 1044 & 25.9 & 107.4 & .. & .. & .. \\
\hline $1920-22$ & 125 & 64 & 61 & 1046 & 25.6 & 105.9 & .. & .. & .. \\
\hline $1930-32$ & 93 & 47 & 45 & 1040 & 19.1 & 78.8 & .. & .. & .. \\
\hline $1940-42$ & 89 & 46 & 43 & 1051 & 18.5 & 73.7 & .. & 4.0 & .. \\
\hline $1950-52$ & 91 & 47 & 44 & 1060 & 17.9 & 81.4 & 2.41 & 2.0 & 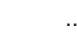 \\
\hline $1960-62$ & 102 & 53 & 50 & 1060 & 19.7 & 97.8 & 2.98 & 2.2 & 20.8 \\
\hline $1970-72$ & 84 & 43 & 41 & 1057 & 16.1 & 83.3 & 2.46 & 1.1 & 13.5 \\
\hline $1980-82$ & 68 & 35 & 33 & 1051 & 13.1 & 62.2 & 1.80 & 0.4 & 6.3 \\
\hline $1990-92$ & 66 & 34 & 32 & 1052 & 13.0 & 59.2 & 1.68 & 0.4 & 5.7 \\
\hline \multirow[t]{2}{*}{$2000-02$} & 52 & 27 & 26 & 1046 & 10.3 & 48.6 & 1.48 & 0.3 & 5.6 \\
\hline & BBCD & KMEU & KMEV & KMEW & KMEX & KMEY & KMEZ & KMFM & KMFN \\
\hline 1998 & 57.3 & 29 & 28 & 1060 & 11.3 & 52.7 & 1.55 & 0.4 & 6.1 \\
\hline 1999 & 55.1 & 28 & 27 & 1050 & 10.9 & 50.9 & 1.51 & 0.3 & 5.2 \\
\hline 2000 & 53.1 & 27 & 26 & 1051 & 10.5 & 49.2 & 1.48 & 0.3 & 5.6 \\
\hline 2001 & 52.5 & 27 & 26 & 1041 & 10.4 & 48.8 & 1.49 & 0.3 & 5.7 \\
\hline 2002 & 51.3 & 26 & 25 & 1047 & 10.1 & 48.1 & 1.48 & 0.3 & 5.4 \\
\hline 2003 & 52.4 & 27 & 26 & 1054 & 10.4 & 49.4 & 1.54 & 0.3 & 5.6 \\
\hline 2004 & 54.0 & 28 & 26 & 1060 & 10.6 & 51.0 & 1.60 & 0.3 & 5.8 \\
\hline 2005 & 54.4 & 28 & 26 & 1068 & 10.7 & 51.5 & 1.62 & 0.3 & 5.3 \\
\hline 2006 & 55.7 & 28 & 27 & 1046 & 10.9 & 52.8 & 1.67 & 0.3 & 5.3 \\
\hline 2007 & 57.8 & 30 & 28 & 1057 & 11.2 & 54.8 & 1.73 & 0.3 & 5.6 \\
\hline 2008 & 60.0 & 31 & 29 & 1037 & 11.6 & 57.2 & 1.80 & 0.3 & 5.4 \\
\hline
\end{tabular}

Northern Ireland ${ }^{7}$

$1900-02$

$1910-12$

$1920-22$

$1930-32$

$1940-42$

$1950-52$

$1960-62$

$1970-72$

$1980-82$

$1990-92$

$2000-02$

31
26
27
29
31
31
28
26
22

$\begin{array}{rr}16 & 1 \\ 13 & 1 \\ 14 & 13 \\ 15 & 1 \\ 16 & 1 \\ 16 & 1 \\ 14 & 13 \\ 13 & 1 \\ 11 & 11\end{array}$

$\begin{array}{rr}. . & \\ 15 & \\ 12 & 1048 \\ 13 & 1047 \\ 14 & 1078 \\ 15 & 1066 \\ 15 & 1068 \\ 13 & 1074 \\ 13 & 1048 \\ 11 & 1051 \\ & 1054\end{array}$

..
24.2
20.5
20.8
20.9
22.5
20.4
18.0
16.1
12.8

105.9

047

1078
1066

1068

074

1051
1054

BBCE

1998

1999

2000

2002

23.7

23.0

21.5

22.0

KMFO

KMFP

KMFQ

1039

1084

1070

1035

KMFR

14.1
13.7

13.7
12.8

13.0

12.6

78.8
73.7

73.7
81.4

111.5

105.7

87.5

74.8

2003

2004

2005

21.6

22.3

22.3

2006

23.3
24.5

2008

25.6

65.0
62.9
58.7
59.7
58.1

59.0
60.6
60.4
62.5
65.1
68.2

KMFT

1.90

1.86

1.75

1.80

KMFU

KMFV

$0.1 \quad 5.1$

$\begin{array}{ll}0.1 & 5.7 \\ 0.1 & 4.3\end{array}$

0.1
0.1

0.1

1081

1059
1032

12.7

13.0
12.9

12.9

1.77

5.7

1066

14.0
13.9

1063

14.4

68.2

1.81
1.87

1.87

2.02

0.1

0.1

5.0

1 See chapter text.

dead after 24 completed weeks gestation or more. Between 1 October and 31 December 1992 in the UK there were 258 babies born dead between 24 and 27

2 Males per 1,000 females (calculated using whole numbers). completed weeks gestation (216 in England and Wales, 35 in Scotland and 7 in

3 Rate per 1,000 population (calculated using whole numbers).

4 Rate per 1,000 women aged $15-44$

5 Total fertility rate is the average number of children which would be born to a woman if she experienced the age-specific fertility rates of the period in question throughout her child-bearing life span. UK figures for the years Northern Ireland). If these babies were included in the stillbirth figures given, the stillbirth rate would be 4.7 for the UK and England and Wales, while Scotland and Northern Ireland stillbirth rate would remain as stated.

1970-72 and earlier are estimates.

6 On 1 October 1992 the legal definition of a stillbirth was changed from a baby born dead after 28 completed weeks gestation or more to one born

7 From 1981, data for the United Kingdom and Northern Ireland have been revised to exclude births in Northern Ireland to non-residents of Northern Ireland. 


\begin{tabular}{|c|c|c|c|c|c|c|c|c|c|c|c|c|}
\hline & \multicolumn{6}{|c|}{ Inside marriage } & \multicolumn{6}{|c|}{ Outside marriage } \\
\hline & All ages & Under 20 & $20-24$ & $25-29$ & Over 30 & $\begin{array}{r}\text { Mean }^{1} \\
\text { age } \\
\text { (Years) }\end{array}$ & All ages & Under 20 & $20-24$ & $25-29$ & Over 30 & $\begin{array}{r}\text { Mean }^{1} \\
\text { age } \\
\text { (Years) }\end{array}$ \\
\hline \multicolumn{13}{|c|}{ United Kingdom² } \\
\hline 1961 & $\begin{array}{r}\text { KKEY } \\
890\end{array}$ & $\begin{array}{r}\text { KKEZ } \\
55\end{array}$ & $\begin{array}{r}\text { KKFY } \\
273\end{array}$ & $\begin{array}{r}\text { KKFZ } \\
280\end{array}$ & $\begin{array}{r}\text { KKGX } \\
282\end{array}$ & $\begin{array}{r}\text { KKGY } \\
27.7\end{array}$ & $\begin{array}{r}\text { KKGZ } \\
54\end{array}$ & $\begin{array}{r}\text { KKIC } \\
13\end{array}$ & $\begin{array}{r}\text { KKID } \\
17\end{array}$ & $\begin{array}{r}\text { KKIE } \\
10\end{array}$ & $\begin{array}{r}\text { KKIF } \\
13\end{array}$ & $\begin{array}{r}\text { KKIG } \\
25.5\end{array}$ \\
\hline 1971 & 828 & 70 & 301 & 271 & 185 & 26.4 & 74 & 24 & 25 & 13 & 12 & 23.8 \\
\hline 1981 & 640 & 36 & 193 & 231 & 180 & 27.3 & 91 & 30 & 33 & 16 & 13 & 23.4 \\
\hline 1988 & 589 & 16 & 144 & 234 & 195 & 28.2 & 198 & 51 & 76 & 42 & 29 & 24.1 \\
\hline 1989 & 570 & 14 & 130 & 228 & 198 & 28.4 & 207 & 49 & 79 & 46 & 32 & 24.3 \\
\hline 1990 & 576 & 13 & 121 & 233 & 209 & 28.6 & 223 & 51 & 83 & 53 & 37 & 24.5 \\
\hline 1991 & 556 & 10 & 109 & 224 & 213 & 28.9 & 236 & 50 & 87 & 58 & 41 & 24.8 \\
\hline 1992 & 540 & 9 & 98 & 216 & 218 & 29.1 & 241 & 46 & 86 & 62 & 46 & 25.1 \\
\hline 1993 & 520 & 8 & 87 & 204 & 221 & 29.3 & 242 & 44 & 84 & 64 & 50 & 25.4 \\
\hline 1994 & 510 & 7 & 78 & 194 & 231 & 29.6 & 240 & 41 & 80 & 65 & 55 & 25.7 \\
\hline 1995 & 486 & 6 & 69 & 180 & 232 & 29.8 & 246 & 42 & 79 & 66 & 60 & 25.9 \\
\hline 1996 & 473 & 6 & 61 & 170 & 237 & 30.1 & 260 & 45 & 80 & 69 & 66 & 26.0 \\
\hline 1997 & 460 & 6 & 55 & 159 & 240 & 30.3 & 267 & 47 & 79 & 71 & 71 & 26.1 \\
\hline 1998 & 447 & 6 & 51 & 149 & 243 & 30.5 & 270 & 49 & 77 & 70 & 74 & 26.2 \\
\hline 1999 & 428 & 6 & 47 & 136 & 239 & 30.7 & 272 & 49 & 77 & 68 & 77 & 26.3 \\
\hline 2000 & 411 & 5 & 44 & 126 & 237 & 30.9 & 268 & 47 & 77 & 66 & 78 & 26.4 \\
\hline 2001 & 401 & 5 & 44 & 116 & 236 & 30.9 & 268 & 45 & 77 & 64 & 82 & 26.7 \\
\hline 2002 & 397 & 5 & 44 & 109 & 239 & 31.1 & 272 & 44 & 80 & 62 & 85 & 26.7 \\
\hline 2003 & 407 & 5 & 44 & 110 & 249 & 31.2 & 289 & 45 & 86 & 65 & 92 & 26.8 \\
\hline 2004 & 413 & 4 & 44 & 110 & 255 & 31.3 & 303 & 47 & 90 & 69 & 97 & 26.9 \\
\hline 2005 & 412 & 4 & 43 & 111 & 254 & 31.4 & 310 & 47 & 93 & 72 & 98 & 26.9 \\
\hline 2006 & 422 & 3 & 43 & 115 & 260 & 31.4 & 327 & 48 & 99 & 78 & 102 & 26.9 \\
\hline 2007 & 429 & 3 & 42 & 119 & 265 & 31.5 & 343 & 47 & 104 & 85 & 106 & 27.1 \\
\hline 2008 & 434 & 3 & 41 & 123 & 266 & 31.5 & 361 & 48 & 110 & 93 & 110 & 27.0 \\
\hline
\end{tabular}

Great Britain

\begin{tabular}{|c|c|c|c|c|c|c|c|c|c|c|c|c|}
\hline & $\mathrm{KKIH}$ & KKII & KKIJ & KKIK & KKIL & KKIM & KKIN & KKIO & KKIP & $\mathrm{KKIQ}$ & KKIR & KKIS \\
\hline 1961 & 859 & 53 & 264 & 270 & 272 & 27.7 & 53 & 13 & 17 & 10 & 13 & 25.5 \\
\hline 1971 & 797 & 68 & 293 & 261 & 176 & 26.4 & 73 & 24 & 25 & 13 & 12 & 23.8 \\
\hline 1988 & 566 & 16 & 138 & 226 & 186 & 28.2 & 194 & 49 & 74 & 42 & 29 & 23.6 \\
\hline 1989 & 549 & 13 & 125 & 220 & 190 & 28.4 & 202 & 48 & 77 & 45 & 32 & 24.2 \\
\hline 1990 & 554 & 12 & 116 & 225 & 201 & 28.6 & 218 & 49 & 81 & 52 & 36 & 24.6 \\
\hline 1992 & 520 & 9 & 94 & 208 & 210 & 29.1 & 235 & 45 & 84 & 61 & 46 & 25.1 \\
\hline 1993 & 500 & 7 & 84 & 196 & 213 & 29.3 & 236 & 42 & 82 & 62 & 49 & 25.4 \\
\hline 1994 & 492 & 7 & 75 & 188 & 222 & 29.6 & 235 & 41 & 78 & 63 & 53 & 25.7 \\
\hline 1995 & 468 & 6 & 66 & 173 & 223 & 29.8 & 240 & 40 & 77 & 65 & 59 & 25.9 \\
\hline 1996 & 455 & 6 & 59 & 163 & 227 & 30.1 & 254 & 44 & 78 & 68 & 65 & 26.0 \\
\hline 1997 & 442 & 6 & 53 & 152 & 231 & 30.3 & 261 & 46 & 76 & 69 & 69 & 26.2 \\
\hline 1998 & 430 & 6 & 49 & 143 & 233 & 30.5 & 263 & 48 & 74 & 68 & 73 & 26.3 \\
\hline 2001 & 386 & 5 & 43 & 112 & 227 & 30.9 & 261 & 44 & 75 & 62 & 80 & 26.6 \\
\hline 2002 & 383 & 5 & 43 & 105 & 230 & 31.1 & 265 & 43 & 77 & 61 & 84 & 26.7 \\
\hline 2003 & 393 & 4 & 43 & 106 & 239 & 31.2 & 281 & 44 & 83 & 64 & 90 & 26.9 \\
\hline 2004 & 399 & 4 & 43 & 106 & 245 & 31.3 & 295 & 45 & 88 & 67 & 95 & 26.9 \\
\hline 2005 & 398 & 4 & 42 & 107 & 245 & 31.4 & 302 & 45 & 90 & 70 & 97 & 26.9 \\
\hline 2006 & 407 & 3 & 42 & 111 & 251 & 31.4 & 318 & 46 & 96 & 76 & 100 & 26.9 \\
\hline 2007 & 414 & 3 & 41 & 114 & 255 & 31.5 & 334 & 46 & 101 & 83 & 104 & 27.1 \\
\hline 2008 & 418 & 3 & 41 & 119 & 256 & 31.5 & 351 & 46 & 107 & 90 & 107 & 27.0 \\
\hline
\end{tabular}

1 The mean ages presented in this table are unstandardised and therefore take no account of the age structure of the population.

2 From 1981, data for the United Kingdom have been revised to exclude

Sources: Office for National Statistics: 01329 444410; General Register Office for Scotland; births in Northern Ireland to non-residents of Northern Ireland. 
5 Live births: by age of mother and country

Numbers

All ages $10020-29$

United Kingdom

All live births ${ }^{1,2}$

$\begin{array}{rr} & \text { KMBZ } \\ 1998 & 716888 \\ 1999 & 699976 \\ 2000 & 679029 \\ 2001 & 669123 \\ 2002 & 668777 \\ 2003 & \\ 2004 & 695549 \\ 2005 & 715996 \\ 2006 & 722549 \\ 2007 & 748563 \\ 2008 & 772245 \\ & 794383\end{array}$

Age-specific fertility rates ${ }^{3}$

$\begin{array}{lr} & \text { KMB } \\ 1998 & 58 . \\ 1999 & 57 \\ 2000 & 55 \\ 2001 & 54 \\ 2002 & 54 \\ & \\ 2003 & 56 \\ 2004 & 57 . \\ 2005 & 57.8 \\ 2006 & 59.7 \\ 2007 & 61 . \\ 2008 & 63.4\end{array}$

England and Wales

All live births

$\begin{array}{lr} & \text { KGSH } \\ 1998 & 635901 \\ 1999 & 621872 \\ 2000 & 604441 \\ 2001 & 594634 \\ 2002 & 596122 \\ 2003 & \\ 2004 & 621469 \\ 2005 & 639721 \\ 2006 & 645835 \\ 2007 & 669601 \\ 2008 & 690013 \\ & 708711\end{array}$

Age-specific fertility rates $^{3}$

MBY
58.8
57.3
55.4
54.3
54.2
56.2
57.7
57.8
59.7
61.5
63.4

$\begin{array}{lr}\text { KMDV } & \text { KMDW } \\ 54822 & 127230 \\ 54921 & 124036 \\ 52059 & 120305 \\ 50157 & 121664 \\ 49165 & 123844 \\ & \\ 49874 & 129867 \\ 50752 & 134614 \\ 50396 & 135891 \\ 51066 & 142171 \\ 50515 & 145725 \\ 50396 & 151608\end{array}$

KMDX
218072
204808
191583
179776
171852
175473
179050
183513
192800
204276
216466

KMDY
212876
208986
202893
202017
203261
210071
213620
211076
212333
214020
215964

KMDZ
88729
91272
95400
97379
101379
109038
114852
116902
123867
129599
130520

KMES
14453
15210
16032
17271
18273
20233
22107
23518
24999
26633
27845

KMET
640
695
708
831
968

933
975
1176
1288
1420
1558

KMBR
30.8

30.8
30.7

29.2

27.9
27.0

26.7

26.7

26.2

26.4
25.9

26.0

$\begin{array}{rr}\text { KMBS } & \text { KMBT } \\ 73.6 & 101.4 \\ 71.8 & 98.0 \\ 68.7 & 93.9 \\ 68.0 & 91.5 \\ 68.0 & 91.3 \\ & \\ 70.2 & 95.4 \\ 71.5 & 97.3 \\ 70.4 & 97.4 \\ 72.0 & 100.1 \\ 72.3 & 103.5 \\ 73.5 & 107.9\end{array}$
KMBU
90.4
89.4
89.4
87.7
87.7
88.0
89.7
94.6
94.6
99.2
100.5
104.6
109.8

113.1

KMBV
40.0
40.2
41.0
41.3
42.6

45.9
48.6
50.0
53.4
56.6

KMBW

7.4
7.6
7.8
8.2
8.4

KMBX

0.3

0.4

0.4
0.5

0.5

0.5

0.6

0.6
0.7

0.7

$\begin{array}{ll}58.2 & 12.4\end{array}$

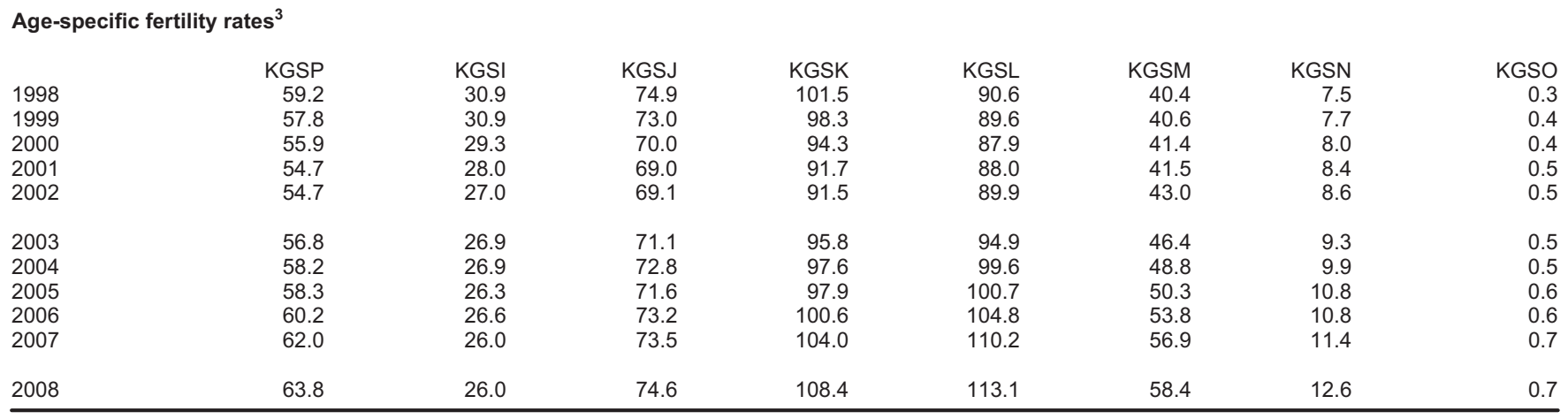


All live births ${ }^{1}$

\begin{tabular}{lr} 
& KGTH \\
1998 & 57319 \\
1999 & 55147 \\
2000 & 53076 \\
2001 & 52527 \\
2002 & 51270 \\
2003 & \\
2004 & 52432 \\
2005 & 53957 \\
2006 & 54386 \\
2007 & 55690 \\
2008 & 57781 \\
& \\
\hline
\end{tabular}

KGTA
4802
4755
4599
4444
4195
4155
4172
4171
4130
4305
4279

KGTB
9804
9440
8962
9121
9267
9626
9950
10008
10399
10913
11373

KGTC
17477
16011
14676
13763
12694
12725
13131
13229
13876
14917
16171

KGTD
17207
16722
16233
16206
16038
16085
16085
15962
15878
15622
16028

KGTE
6893
7034
7395
7701
7727
8310
8912
9179
9535
10035
10025

$$
\begin{array}{r}
\text { KGTF } \\
1027 \\
1096 \\
1133 \\
1224 \\
1267 \\
1432 \\
1631 \\
1694 \\
1775 \\
1849 \\
2044
\end{array}
$$

KGTG

\begin{tabular}{|c|c|c|c|}
\hline & KGTP & KGTI & KGTJ \\
\hline 1998 & 52.7 & 30.6 & 62.8 \\
\hline 1999 & 50.9 & 30.3 & 61.0 \\
\hline 2000 & 49.2 & 29.3 & 57.6 \\
\hline 2001 & 48.8 & 28.4 & 57.8 \\
\hline 2002 & 48.1 & 26.8 & 58.3 \\
\hline 2003 & 49.4 & 26.3 & 60.1 \\
\hline 2004 & 51.0 & 26.1 & 61.8 \\
\hline 2005 & 51.5 & 26.2 & 60.9 \\
\hline 2006 & 52.8 & 25.8 & 61.9 \\
\hline 2007 & 54.8 & 26.9 & 63.6 \\
\hline 2008 & 57.2 & 26.8 & 65.3 \\
\hline
\end{tabular}

Age-specific fertility rates ${ }^{3}$

KGTK
94.3
90.4
86.5
85.1
83.3
86.5
89.4
88.6
90.2
93.1
98.1

$$
\begin{array}{r}
\text { KGTL } \\
83.2 \\
82.0 \\
81.3 \\
82.2 \\
83.6 \\
\\
86.8 \\
90.3 \\
93.2 \\
97.1 \\
100.1
\end{array}
$$$$
\begin{aligned}
& 82.2 \\
& 83.6
\end{aligned}
$$$$
93.2
$$

KGTM

34.1

35.6
36.9

37.1

40.0

43.3

45.4

47.8
51.3

105.4

53.1

KGTN
5.7
5.9
6.0
6.3
6.4

7.1
7.9
8.1
8.4
8.8

KGTO

0.3
0.2

Northern Ireland

All live births ${ }^{2}$

\begin{tabular}{|c|c|c|c|}
\hline & KMDU & KMDN & KMDO \\
\hline 1998 & 65.0 & 27.8 & 69.6 \\
\hline 1999 & 62.9 & 28.6 & 70.6 \\
\hline 2000 & 58.7 & 25.6 & 66.0 \\
\hline 2001 & 59.7 & 23.9 & 67.5 \\
\hline 2002 & 58.1 & 23.3 & 66.0 \\
\hline 2003 & 59.0 & 22.9 & 65.5 \\
\hline 2004 & 60.6 & 23.0 & 62.8 \\
\hline 2005 & 60.4 & 21.7 & 63.2 \\
\hline 2006 & 62.5 & 22.5 & 63.6 \\
\hline 2007 & 65.1 & 22.5 & 62.6 \\
\hline 2008 & 68.2 & 23.0 & 65.6 \\
\hline
\end{tabular}

$\begin{array}{ll} & \text { KMDM } \\ 1998 & 23668 \\ 1999 & 22957 \\ 2000 & 21512 \\ 2001 & 21962 \\ 2002 & 21385 \\ 2003 & \\ 2004 & 21648 \\ 2005 & 22318 \\ 2006 & 22328 \\ 2007 & 23272 \\ 2008 & 24451 \\ & 25631\end{array}$

KMDF
1735
1791
1614
1524
1502
1483
1486
1395
1427
1405
1426

KMDG
3889
3874
3602
3699
3619
3619
3592
3738
3944
4028
4264

KMDH
7451
6866
6206
6087
5779
5817
5935
5936
6282
6789
7335

$\begin{array}{lr}\text { KMDI } & \text { KMDJ } \\ 7170 & 2955 \\ 6953 & 2957 \\ 6547 & 3031 \\ 6891 & 3183 \\ 6691 & 3203 \\ 6772 & 3342 \\ 6985 & 3712 \\ 6961 & 3610 \\ 7048 & 3823 \\ 7274 & 4184 \\ 7486 & 4275\end{array}$

KMDK
446
497
496
548
565
596
592
669
712
741
810

KMDL

Age-specific fertility rates ${ }^{2,3}$

1 The 'All ages' figure for Scotland includes births to mothers whose age was not known. There were 66 such births in 1998, 48 in 1999, 49 in 2000, 28 in 2001,35 in 2002, 60 in 2003, 26 in 2004,

77 in 2005, 39 in 2006, 57 in 2007 and 26 in 2008.

\begin{tabular}{rrrrr} 
KMDP & KMDQ & KMDR & KMDS & KMDT \\
119.0 & 108.4 & 47.2 & 8.2 & 0.4 \\
112.3 & 105.6 & 46.1 & 8.9 & 0.4 \\
103.9 & 100.4 & 46.2 & 8.5 & 0.3 \\
105.1 & 106.0 & 48.0 & 9.1 & 0.6 \\
102.9 & 104.2 & 48.2 & 9.2 & 0.5 \\
106.8 & & & & 0.3 \\
109.8 & 107.0 & 50.2 & 9.8 & 0.3 \\
108.6 & 112.6 & 56.1 & 9.5 & 0.3 \\
112.0 & 114.8 & 55.0 & 10.2 & 0.6 \\
116.1 & 119.2 & 58.2 & 10.8 & 0.5 \\
120.5 & 124.6 & 64.2 & 11.1 & 0.6 \\
\hline
\end{tabular}

Sources: Office for National Statistics: 01329 444410; General Register Office for Scotland; Northern Ireland Statistics and Research Agency

2 From 1981 data for the United Kingdom and Northern Ireland have been revised to exclude births in Northern Ireland to non residents in Northern Ireland.

3 The rates for women of all ages, under 20 , and 45 and over are based upon the populations of women aged $15-44,15-19$ and 45 respectively. 


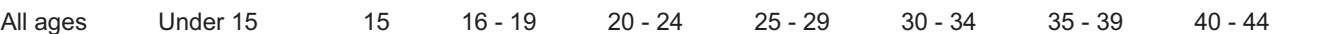

England and Wales

\begin{tabular}{|c|c|c|c|c|c|c|c|c|c|c|c|}
\hline & C53Z & C542 & C543 & C544 & C545 & C546 & C547 & C548 & C549 & C54A & C54B \\
\hline 1987 & 156191 & 907 & 2858 & 35167 & 49256 & 31243 & 18960 & 12639 & 4757 & 390 & 14 \\
\hline 1988 & 168298 & 859 & 2709 & 37928 & 54067 & 34584 & 20000 & 12681 & 5047 & 412 & 11 \\
\hline 1989 & 170463 & 803 & 2580 & 36182 & 54880 & 36604 & 21284 & 12713 & 5020 & 388 & 9 \\
\hline 1990 & 173900 & 873 & 2549 & 35520 & 55281 & 38770 & 22431 & 12956 & 5104 & 404 & 12 \\
\hline 1991 & 167376 & 886 & 2272 & 31130 & 52678 & 38611 & 23445 & 13035 & 4901 & 408 & 10 \\
\hline 1992 & 160501 & 905 & 2095 & 27589 & 49052 & 38430 & 23870 & 13252 & 4844 & 452 & 12 \\
\hline 1993 & 157846 & 964 & 2119 & 25806 & 46846 & 38139 & 24690 & 13885 & 4889 & 494 & 14 \\
\hline 1994 & 156539 & 1080 & 2166 & 25223 & 44871 & 38081 & 25507 & 14156 & 5008 & 440 & 7 \\
\hline 1996 & 167916 & 1098 & 2547 & 28790 & 46356 & 39311 & 28228 & 16118 & 5027 & 428 & 13 \\
\hline 1997 & 170145 & 1020 & 2414 & 29947 & 44960 & 40159 & 28892 & 16858 & 5413 & 482 & \\
\hline 1998 & 177871 & 1103 & 2656 & 33236 & 45766 & 40366 & 30449 & 18174 & 5576 & 511 & 34 \\
\hline 1999 & 173701 & 1066 & 2537 & 32807 & 45004 & 38492 & 29139 & 18341 & 5755 & 502 & 58 \\
\hline 2000 & 175542 & 1048 & 2700 & 33218 & 47099 & 37852 & 28735 & 18589 & 5794 & 459 & 48 \\
\hline 2001 & 176364 & 1066 & 2592 & 33431 & 48267 & 36506 & 28782 & 19146 & 6094 & 456 & 24 \\
\hline 2002 & 175932 & 1075 & 2658 & 32985 & 48359 & 35795 & 28503 & 19450 & 6531 & 457 & 119 \\
\hline 2003 & 181582 & 1171 & 2796 & 34247 & $51201^{2}$ & 36018 & 28749 & 19868 & 7032 & 500 & - \\
\hline 2005 & 186416 & 1083 & 2703 & 35313 & 53342 & 38330 & 27836 & 19782 & 7459 & 568 & - \\
\hline 2006 & 193737 & 1042 & 2948 & 37296 & 55340 & 40396 & 28153 & 20074 & 7825 & 663 & - \\
\hline 2007 & 198499 & 1171 & 3205 & 39579 & 56963 & 41704 & 27257 & 19976 & 7915 & 729 & - \\
\hline 2008 & 195296 & 1097 & 3016 & 38577 & 56171 & 41896 & 26985 & 19228 & 7663 & 663 & - \\
\hline
\end{tabular}

Scotland

\begin{tabular}{|c|c|c|c|c|c|c|c|c|c|c|c|}
\hline & C54C & C54D & C54E & $\mathrm{C} 54 \mathrm{~F}$ & C54G & $\mathrm{C} 54 \mathrm{H}$ & C54I & C54J & C54K & C54L & EVH4 \\
\hline 1987 & 9449 & 70 & 210 & 2415 & 2991 & 1728 & 1082 & 695 & 241 & 17 & - \\
\hline 1988 & 10111 & 65 & 217 & 2526 & 3299 & 1965 & 1105 & 662 & 257 & 15 & - \\
\hline 1989 & 10191 & 53 & 209 & 2554 & 3199 & 1967 & 1225 & 704 & 266 & 14 & - \\
\hline 1990 & 10198 & 54 & 185 & 2536 & 3235 & 2061 & 1157 & 698 & 253 & 19 & - \\
\hline 1992 & 10791 & 73 & 173 & 2368 & 3383 & 2283 & 1444 & 798 & 252 & 17 & - \\
\hline 1993 & 11059 & 92 & 193 & 2297 & 3365 & 2443 & 1489 & 889 & 262 & 29 & - \\
\hline 1994 & 11371 & 78 & 214 & 2311 & 3480 & 2427 & 1640 & 876 & 315 & 30 & - \\
\hline 1996 & 11957 & 87 & 234 & 2360 & 3569 & 2595 & 1798 & 957 & 330 & 27 & - \\
\hline 1997 & 12087 & 85 & 204 & 2429 & 3438 & 2644 & 1849 & 1091 & 322 & 25 & - \\
\hline 1998 & 12458 & 73 & 213 & 2703 & 3419 & 2740 & 1801 & 1148 & 339 & 22 & - \\
\hline 1999 & 12140 & 69 & 182 & 2628 & 3349 & 2546 & 1807 & 1178 & 358 & 23 & - \\
\hline 2000 & 11976 & 93 & 181 & 2606 & 3348 & 2400 & 1765 & 1174 & 381 & 28 & - \\
\hline 2001 & 12108 & 66 & 210 & 2717 & 3459 & 2315 & 1815 & 1126 & 377 & 23 & - \\
\hline 2002 & 11840 & 80 & 194 & 2646 & 3447 & 2165 & 1731 & 1166 & 382 & 29 & - \\
\hline 2003 & 12267 & 71 & 242 & 2781 & 3673 & 2224 & 1725 & 1112 & 411 & 28 & _ \\
\hline 2005 & 12605 & 94 & 248 & 2966 & 3765 & 2334 & 1682 & 1094 & 397 & 25 & - \\
\hline 2006 & 13109 & 91 & 273 & 3085 & 3967 & 2433 & 1614 & 1205 & 413 & 28 & - \\
\hline 2007 & 13672 & 98 & 275 & 3170 & 4108 & 2722 & 1636 & 1219 & 407 & 37 & - \\
\hline $2008^{3}$ & 13762 & 94 & 247 & 3125 & 4259 & 2736 & 1641 & 1204 & 427 & 29 & - \\
\hline
\end{tabular}

1 Refers to therapeutic abortions notified in accordance with the Abortion Act Sources: Department of Health;

2 Records with missing ages were assigned to the $20-24$ age group. Notifications (to the Chief Medical Officer for Scotland) of abortions performed; 3 Provisional. 
Population and vital statistics

5. 19 Deaths: by sex and age ${ }^{1}$

Numbers

\begin{tabular}{|c|c|c|c|c|c|c|c|c|c|c|c|c|c|c|}
\hline & $\begin{array}{r}\text { All } \\
\text { ages }^{2}\end{array}$ & $\begin{array}{l}\text { Under } \\
1 \text { year }\end{array}$ & $1-4$ & $5-9$ & $10-14$ & $15-19$ & $20-24$ & $25-34$ & $35-44$ & $45-54$ & $55-64$ & $65-74$ & $75-84$ & $\begin{array}{r}85 \text { and } \\
\text { over }\end{array}$ \\
\hline \multicolumn{15}{|c|}{ United Kingdom } \\
\hline \multicolumn{15}{|l|}{ Males } \\
\hline $1900-02$ & 340664 & 87242 & 37834 & 8429 & 4696 & 7047 & 8766 & 19154 & 24739 & 30488 & 37610 & 39765 & 28320 & 6563 \\
\hline $1910-12$ & 303703 & 63885 & 29452 & 7091 & 4095 & 5873 & 6817 & 16141 & 21813 & 28981 & 37721 & 45140 & 29397 & 7283 \\
\hline 1920 - 22 & 284876 & 48044 & 19008 & 6052 & 3953 & 5906 & 6572 & 13663 & 19702 & 29256 & 40583 & 49398 & 34937 & 7801 \\
\hline $1930-32$ & 284249 & 28840 & 11276 & 4580 & 2890 & 5076 & 6495 & 12327 & 16326 & 29376 & 47989 & 63804 & 45247 & 10022 \\
\hline $1940-42$ & 314643 & 24624 & 6949 & 3400 & 2474 & 4653 & 4246 & 11506 & 17296 & 30082 & 57076 & 79652 & 59733 & 12900 \\
\hline $1950-52$ & 307312 & 14105 & 2585 & 1317 & 919 & 1498 & 2289 & 5862 & 11074 & 27637 & 53691 & 86435 & 79768 & 20131 \\
\hline $1960-62$ & 318850 & 12234 & 1733 & 971 & 871 & 1718 & 1857 & 3842 & 8753 & 26422 & 63009 & 87542 & 83291 & 26605 \\
\hline $1970-72$ & 335166 & 9158 & 1485 & 1019 & 802 & 1778 & 2104 & 3590 & 7733 & 24608 & 64898 & 105058 & 82905 & 30027 \\
\hline 1980 - 82 & 330495 & 4829 & 774 & 527 & 652 & 1999 & 1943 & 3736 & 6568 & 19728 & 54159 & 105155 & 98488 & 31936 \\
\hline 1990 - 92 & 312521 & 3315 & 623 & 372 & 396 & 1349 & 2059 & 4334 & 6979 & 15412 & 40424 & 87849 & 106376 & 43032 \\
\hline \multirow[t]{2}{*}{$2000-02$} & 288261 & 2065 & 365 & 233 & 326 & 1032 & 1502 & 4270 & 7181 & 15370 & 32328 & 66808 & 98363 & 58419 \\
\hline & KHUA & KHUB & KHUC & KHUD & KHUE & KHUF & KHUG & KHUH & KHUI & KHUJ & KHUK & KHUL & KHUM & KHUN \\
\hline 1997 & 301713 & 2414 & 465 & 301 & 366 & 1134 & 1738 & 4558 & 6678 & 15770 & 33910 & 78121 & 101817 & 54441 \\
\hline 1999 & 299235 & 2323 & 459 & 260 & 333 & 1088 & 1548 & 4647 & 6930 & 15862 & 33181 & 73457 & 101327 & 57820 \\
\hline 2000 & 291337 & 2136 & 390 & 263 & 305 & 1068 & 1595 & 4491 & 7168 & 15458 & 32661 & 69707 & 98398 & 57697 \\
\hline 2001 & 287942 & 2052 & 358 & 230 & 369 & 1106 & 1518 & 4459 & 7275 & 15668 & 32135 & 66257 & 98041 & 58474 \\
\hline 2002 & 289083 & 2050 & 382 & 224 & 327 & 1071 & 1575 & 4345 & 7362 & 15222 & 32509 & 65140 & 99387 & 59489 \\
\hline 2003 & 289185 & 2047 & 356 & 228 & 308 & 1013 & 1586 & 4041 & 7530 & 14692 & 32895 & 63520 & 100900 & 60069 \\
\hline 2004 & 278918 & 2033 & 345 & 206 & 282 & 975 & 1484 & 3831 & 7454 & 14510 & 31660 & 60760 & 98466 & 56912 \\
\hline 2005 & 277349 & 2117 & 339 & 194 & 312 & 1022 & 1449 & 3660 & 7454 & 14241 & 31645 & 58828 & 95641 & 60447 \\
\hline 2006 & 274201 & 2078 & 328 & 213 & 299 & 1008 & 1482 & 3712 & 7485 & 14406 & 32012 & 56319 & 92532 & 62327 \\
\hline 2007 & 274883 & 2106 & 379 & 200 & 270 & 979 & 1491 & 3711 & 7423 & 14045 & 31994 & 55338 & 91488 & 65459 \\
\hline 2008 & 276745 & 2123 & 319 & 211 & 218 & 955 & 1487 & 3718 & 7519 & 14420 & 31406 & 55445 & 90367 & 68557 \\
\hline \multicolumn{15}{|l|}{ Females } \\
\hline 1900 - 02 & 322058 & 68770 & 36164 & 8757 & 5034 & 6818 & 8264 & 18702 & 21887 & 25679 & 34521 & 42456 & 34907 & 10099 \\
\hline $1910-12$ & 289608 & 49865 & 27817 & 7113 & 4355 & 5683 & 6531 & 15676 & 19647 & 24481 & 32813 & 46453 & 37353 & 11828 \\
\hline $1920-22$ & 274772 & 35356 & 17323 & 5808 & 4133 & 5729 & 6753 & 14878 & 18121 & 24347 & 34026 & 48573 & 45521 & 14203 \\
\hline 1930 - 32 & 275336 & 21072 & 9995 & 3990 & 2734 & 4721 & 5931 & 12699 & 15373 & 24695 & 39471 & 59520 & 56250 & 18886 \\
\hline $1940-42$ & 296646 & 17936 & 5952 & 2743 & 2068 & 4180 & 5028 & 11261 & 14255 & 23629 & 42651 & 70907 & 71377 & 24658 \\
\hline 1980 - 82 & 330269 & 3561 & 585 & 355 & 425 & 733 & 772 & 2099 & 4360 & 12206 & 32052 & 72618 & 117760 & 82743 \\
\hline 1990 - 92 & 328218 & 2431 & 485 & 259 & 255 & 520 & 714 & 1989 & 4340 & 9707 & 25105 & 61951 & 115467 & 104994 \\
\hline \multirow[t]{2}{*}{$2000-02$} & 317356 & 1586 & 283 & 188 & 208 & 446 & 536 & 1877 & 4426 & 10270 & 20549 & 47324 & 101650 & 128012 \\
\hline & KIUA & KIUB & KIUC & KIUD & KIUE & KIUF & KIUG & $\mathrm{KIUH}$ & IUI & JJ & KIUK & KIUL & KIUM & KIUN \\
\hline 1997 & 330804 & 1863 & 336 & 221 & 236 & 489 & 587 & 1953 & 4320 & 10451 & 21103 & 55947 & 108777 & 124521 \\
\hline 1998 & 327937 & 1744 & 339 & 221 & 233 & 511 & 554 & 2015 & 4316 & 10441 & 20819 & 54048 & 106703 & 125993 \\
\hline 1999 & 330241 & 1736 & 346 & 195 & 244 & 487 & 567 & 1963 & 4359 & 10400 & 20963 & 52098 & 106323 & 130560 \\
\hline 2000 & 319242 & 1677 & 288 & 181 & 215 & 468 & 573 & 1975 & 4488 & 10477 & 20620 & 49138 & 102052 & 127090 \\
\hline 2001 & 316451 & 1639 & 299 & 218 & 200 & 447 & 557 & 1895 & 4475 & 10354 & 20479 & 47138 & 101135 & 127615 \\
\hline 2002 & 318962 & 1488 & 280 & 181 & 229 & 456 & 556 & 1838 & 4380 & 10080 & 20707 & 46094 & 102503 & 130170 \\
\hline 2003 & 322900 & 1639 & 309 & 182 & 237 & 441 & 563 & 1869 & 4506 & 9870 & 20974 & 45374 & 105182 & 131754 \\
\hline 2004 & 305873 & 1626 & 279 & 160 & 201 & 480 & 572 & 1765 & 4486 & 9463 & 20500 & 43118 & 100775 & 122448 \\
\hline 2005 & 305615 & 1555 & 256 & 153 & 216 & 450 & 557 & 1684 & 4432 & 9492 & 20655 & 41839 & 98338 & 125988 \\
\hline 2006 & 298023 & 1659 & 303 & 151 & 201 & 437 & 520 & 1604 & 4434 & 9474 & 20855 & 40290 & 92877 & 125218 \\
\hline 2007 & 299797 & 1627 & 266 & 137 & 212 & 423 & 522 & 1575 & 4398 & 9487 & 21026 & 39685 & 90822 & 129617 \\
\hline 2008 & 302952 & 1622 & 296 & 158 & 161 & 430 & 544 & 1667 & 4446 & 9680 & 21060 & 39496 & 89459 & 133933 \\
\hline
\end{tabular}




\begin{tabular}{|c|c|c|c|c|c|c|c|c|c|c|c|c|c|c|}
\hline & $\begin{array}{r}\text { All } \\
\text { ages }^{2}\end{array}$ & $\begin{array}{l}\text { Under } \\
1 \text { year }\end{array}$ & $1-4$ & $5-9$ & $10-14$ & $15-19$ & $20-24$ & $25-34$ & $35-44$ & $45-54$ & $55-64$ & $65-74$ & $75-84$ & $\begin{array}{r}85 \text { and } \\
\text { over }\end{array}$ \\
\hline \multicolumn{15}{|c|}{ England and Wales } \\
\hline \multicolumn{15}{|l|}{ Males } \\
\hline $1900-02$ & 288886 & 76095 & 32051 & 7066 & 3818 & 5611 & 7028 & 15869 & 21135 & 26065 & 31600 & 33568 & 23835 & 5144 \\
\hline $1910-12$ & 257253 & 54678 & 24676 & 5907 & 3348 & 4765 & 5596 & 13603 & 18665 & 24820 & 32217 & 38016 & 24928 & 6036 \\
\hline $1920-22$ & 240605 & 39796 & 15565 & 5151 & 3314 & 4901 & 5447 & 11551 & 17004 & 25073 & 34639 & 42025 & 29685 & 6455 \\
\hline $1930-32$ & 243147 & 23331 & 9099 & 3844 & 2435 & 4354 & 5580 & 10600 & 14041 & 25657 & 41581 & 54910 & 39091 & 8624 \\
\hline $1940-42$ & 268876 & 19393 & 5616 & 2834 & 2051 & 3832 & 3156 & 9484 & 14744 & 25983 & 50058 & 68791 & 51779 & 11158 \\
\hline $1950-52$ & 266879 & 11498 & 2131 & 1087 & 778 & 1248 & 1947 & 4990 & 9489 & 23815 & 46948 & 75774 & 69496 & 17677 \\
\hline $1960-62$ & 278369 & 10157 & 1444 & 812 & 742 & 1523 & 1624 & 3278 & 7524 & 22813 & 54908 & 77000 & 73180 & 23364 \\
\hline $1970-72$ & 293934 & 7818 & 1259 & 860 & 677 & 1524 & 1788 & 3079 & 6637 & 21348 & 56667 & 92389 & 73365 & 26522 \\
\hline $1980-82$ & 290352 & 4168 & 657 & 452 & 555 & 1716 & 1619 & 3169 & 5590 & 16909 & 47144 & 92485 & 87338 & 28551 \\
\hline $1990-92$ & 275550 & 2926 & 545 & 325 & 338 & 1157 & 1757 & 3717 & 6057 & 13258 & 34977 & 77063 & 94672 & 38757 \\
\hline \multirow[t]{2}{*}{$2000-02$} & 253706 & 1836 & 323 & 200 & 282 & 862 & 1244 & 3619 & 6104 & 13184 & 27696 & 58114 & 87481 & 52761 \\
\hline & KHVA & KHVB & KHVC & KHVD & KHVE & KHVF & KHVG & KHVH & KHVI & KHVJ & KHVK & KHVL & KHVM & KHVN \\
\hline 1997 & 266164 & 2160 & 421 & 268 & 327 & 970 & 1468 & 3915 & 5718 & 13565 & 29110 & 68275 & 90659 & 49308 \\
\hline 1999 & 263166 & 2080 & 408 & 221 & 289 & 905 & 1265 & 3978 & 5918 & 13633 & 28532 & 64017 & 89963 & 51957 \\
\hline 2000 & 256698 & 1902 & 345 & 227 & 263 & 898 & 1328 & 3849 & 6135 & 13355 & 28003 & 60801 & 87449 & 52143 \\
\hline 2001 & 253608 & 1818 & 329 & 192 & 320 & 927 & 1276 & 3830 & 6184 & 13424 & 27599 & 57638 & 87238 & 52833 \\
\hline 2002 & 254390 & 1831 & 329 & 198 & 286 & 912 & 1310 & 3665 & 6255 & 13011 & 27807 & 56584 & 88493 & 53709 \\
\hline 2003 & 254433 & 1827 & 310 & 203 & 263 & 852 & 1348 & 3478 & 6440 & 12697 & 28291 & 55064 & 89596 & 54064 \\
\hline 2004 & 245208 & 1809 & 303 & 174 & 252 & 833 & 1257 & 3281 & 6360 & 12417 & 27117 & 52709 & 87367 & 51329 \\
\hline 2005 & 243870 & 1877 & 297 & 166 & 272 & 856 & 1217 & 3146 & 6362 & 12158 & 27292 & 51019 & 84661 & 54547 \\
\hline 2006 & 240888 & 1863 & 292 & 187 & 261 & 844 & 1212 & 3132 & 6315 & 12256 & 27551 & 48881 & 81912 & 56182 \\
\hline 2007 & 240780 & 1882 & 339 & 182 & 226 & 797 & 1218 & 3138 & 6264 & 11893 & 27508 & 47830 & 80573 & 58930 \\
\hline 2008 & 243014 & 1920 & 284 & 180 & 190 & 779 & 1255 & 3088 & 6419 & 12269 & 27093 & 47862 & 79799 & 61876 \\
\hline \multicolumn{15}{|l|}{ Females } \\
\hline $1900-02$ & 269432 & 60090 & 30674 & 7278 & 4010 & 5265 & 6497 & 15065 & 18253 & 21474 & 28424 & 35307 & 29118 & 7977 \\
\hline $1910-12$ & 242079 & 42642 & 23335 & 5883 & 3519 & 4522 & 5256 & 12742 & 16363 & 20611 & 27571 & 38489 & 31363 & 9782 \\
\hline $1920-22$ & 229908 & 29178 & 14174 & 4928 & 3456 & 4719 & 5533 & 12244 & 15142 & 20580 & 28633 & 41010 & 38439 & 11871 \\
\hline $1930-32$ & 233915 & 16929 & 8013 & 3338 & 2293 & 3969 & 5039 & 10716 & 13022 & 21190 & 33798 & 50844 & 48531 & 16234 \\
\hline $1940-42$ & 253702 & 14174 & 4726 & 2265 & 1695 & 3426 & 4198 & 9470 & 12093 & 20413 & 36814 & 60987 & 61891 & 21550 \\
\hline $1980-82$ & 290026 & 3064 & 511 & 301 & 365 & 635 & 670 & 1821 & 3740 & 10420 & 27606 & 63023 & 103676 & 74194 \\
\hline $1990-92$ & 288851 & 2161 & 420 & 227 & 217 & 455 & 625 & 1718 & 3765 & 8347 & 21466 & 53783 & 101752 & 93914 \\
\hline \multirow[t]{2}{*}{$2000-02$} & 279482 & 1412 & 251 & 168 & 182 & 382 & 455 & 1629 & 3805 & 8893 & 17659 & 40734 & 89387 & 114525 \\
\hline & KIVA & KIVB & KIVC & KIVD & KIVE & KIVF & KIVG & KIVH & IVI & KIVJ & KIVK & KIVL & KIVM & KIVN \\
\hline 1997 & 291888 & 1664 & 300 & 183 & 206 & 428 & 503 & 1711 & 3734 & 9055 & 18053 & 48553 & 96009 & 111489 \\
\hline 1998 & 289233 & 1547 & 301 & 185 & 207 & 432 & 466 & 1768 & 3705 & 9077 & 17872 & 46742 & 94281 & 112650 \\
\hline 1999 & 290366 & 1555 & 308 & 168 & 219 & 399 & 484 & 1707 & 3773 & 8999 & 17949 & 44958 & 93360 & 116487 \\
\hline 2000 & 281179 & 1497 & 257 & 160 & 191 & 403 & 504 & 1702 & 3853 & 9108 & 17722 & 42318 & 89651 & 113813 \\
\hline 2001 & 278890 & 1449 & 272 & 198 & 171 & 386 & 472 & 1665 & 3858 & 8984 & 17608 & 40639 & 89036 & 114152 \\
\hline 2002 & 280966 & 1337 & 240 & 160 & 204 & 391 & 467 & 1597 & 3767 & 8689 & 17807 & 39645 & 90213 & 116449 \\
\hline 2003 & 284718 & 1479 & 278 & 159 & 209 & 370 & 485 & 1636 & 3884 & 8554 & 18001 & 39001 & 92694 & 117968 \\
\hline 2004 & 269042 & 1462 & 251 & 140 & 173 & 410 & 494 & 1536 & 3855 & 8139 & 17649 & 37041 & 88404 & 109488 \\
\hline 2005 & 269123 & 1371 & 222 & 134 & 189 & 379 & 478 & 1481 & 3805 & 8175 & 17797 & 35913 & 86309 & 112870 \\
\hline 2006 & 261711 & 1505 & 267 & 135 & 169 & 381 & 444 & 1382 & 3802 & 8098 & 17948 & 34502 & 81210 & 111868 \\
\hline 2007 & 263265 & 1456 & 235 & 121 & 192 & 357 & 453 & 1360 & 3787 & 8072 & 18166 & 33903 & 79411 & 115752 \\
\hline 2008 & 266076 & 1449 & 270 & 137 & 140 & 351 & 458 & 1445 & 3811 & 8291 & 18187 & 33883 & 77827 & 119827 \\
\hline
\end{tabular}


Population and vital statistics

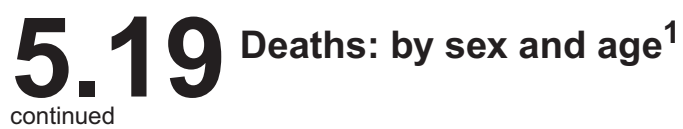

Numbers

\begin{tabular}{|c|c|c|c|c|c|c|c|c|c|c|c|c|c|c|}
\hline & $\begin{array}{r}\text { All } \\
\text { ages }^{2}\end{array}$ & $\begin{array}{l}\text { Under } \\
1 \text { year }\end{array}$ & $1-4$ & $5-9$ & $10-14$ & $15-19$ & $20-24$ & $25-34$ & $35-44$ & $45-54$ & $55-64$ & $65-74$ & $75-84$ & $\begin{array}{r}85 \text { and } \\
\text { over }\end{array}$ \\
\hline \multicolumn{15}{|l|}{ Scotland } \\
\hline \multicolumn{15}{|l|}{ Males } \\
\hline $1900-02$ & 40224 & 9189 & 4798 & 1083 & 672 & 1069 & 1292 & 2506 & 2935 & 3591 & 4597 & 4531 & 3117 & 834 \\
\hline $1910-12$ & 35981 & 7510 & 3935 & 962 & 595 & 826 & 910 & 1969 & 2469 & 3325 & 4356 & 5113 & 3182 & 813 \\
\hline $1920-22$ & 34649 & 6757 & 2847 & 710 & 489 & 747 & 791 & 1616 & 2128 & 3314 & 4785 & 5624 & 3928 & 911 \\
\hline $1940-42$ & 36384 & 3973 & 1011 & 449 & 321 & 668 & 888 & 1643 & 2090 & 3348 & 5728 & 8556 & 6317 & 1337 \\
\hline $1950-52$ & 32236 & 1949 & 349 & 175 & 105 & 200 & 265 & 693 & 1267 & 3151 & 5574 & 8544 & 8094 & 1871 \\
\hline $1960-62$ & 32401 & 1578 & 222 & 121 & 102 & 146 & 185 & 456 & 1013 & 2986 & 6682 & 8505 & 7980 & 2425 \\
\hline $1970-72$ & 32446 & 944 & 168 & 119 & 93 & 178 & 233 & 396 & 875 & 2617 & 6641 & 10176 & 7383 & 2624 \\
\hline $1980-82$ & 31723 & 451 & 80 & 56 & 71 & 206 & 233 & 423 & 776 & 2280 & 5601 & 10152 & 8804 & 2591 \\
\hline $1990-92$ & 29421 & 287 & 57 & 34 & 40 & 137 & 230 & 485 & 744 & 1730 & 4402 & 8611 & 9311 & 3353 \\
\hline \multirow[t]{2}{*}{$2000-02$} & 27526 & 165 & 30 & 23 & 30 & 119 & 196 & 523 & 882 & 1775 & 3781 & 7038 & 8535 & 4430 \\
\hline & KHWA & KHWB & KHWC & KHWD & KHWE & KHWF & KHWG & KHWH & $\mathrm{KHWI}$ & KHWJ & KHWK & KHWL & KHWM & KHWN \\
\hline 1997 & 28305 & 186 & 32 & 22 & 27 & 114 & 208 & 521 & 788 & 1794 & 3876 & 7909 & 8791 & 4037 \\
\hline 1999 & 28605 & 161 & 31 & 23 & 33 & 138 & 215 & 545 & 818 & 1820 & 3773 & 7569 & 8908 & 4571 \\
\hline 2000 & 27511 & 173 & 33 & 24 & 28 & 115 & 198 & 512 & 842 & 1716 & 3789 & 7224 & 8523 & 4334 \\
\hline 2001 & 27324 & 155 & 22 & 27 & 35 & 131 & 179 & 510 & 902 & 1820 & 3751 & 6950 & 8433 & 4409 \\
\hline 2002 & 27743 & 167 & 34 & 17 & 27 & 111 & 211 & 546 & 901 & 1789 & 3804 & 6940 & 8648 & 4548 \\
\hline 2003 & 27832 & 146 & 35 & 15 & 31 & 122 & 186 & 469 & 893 & 1634 & 3787 & 6797 & 8994 & 4723 \\
\hline 2004 & 26775 & 160 & 29 & 21 & 23 & 105 & 181 & 449 & 889 & 1676 & 3629 & 6507 & 8733 & 4373 \\
\hline 2005 & 26522 & 159 & 33 & 19 & 30 & 106 & 150 & 385 & 882 & 1654 & 3478 & 6352 & 8691 & 4583 \\
\hline 2006 & 26251 & 145 & 21 & 18 & 21 & 112 & 206 & 461 & 938 & 1697 & 3567 & 5966 & 8353 & 4746 \\
\hline 2007 & 26895 & 154 & 25 & 13 & 31 & 127 & 207 & 461 & 908 & 1685 & 3572 & 6082 & 8547 & 5083 \\
\hline 2008 & 26504 & 140 & 22 & 19 & 16 & 106 & 174 & 503 & 868 & 1715 & 3481 & 6052 & 8290 & 5118 \\
\hline \multicolumn{15}{|l|}{ Females } \\
\hline $1900-02$ & 39891 & 7143 & 4477 & 1162 & 747 & 1058 & 1246 & 2625 & 2732 & 3130 & 4485 & 5273 & 4305 & 1508 \\
\hline $1910-12$ & 36132 & 5854 & 3674 & 981 & 618 & 836 & 910 & 2149 & 2473 & 2909 & 3960 & 5636 & 4588 & 1552 \\
\hline $1920-22$ & 34449 & 5029 & 2602 & 687 & 489 & 711 & 889 & 1947 & 2266 & 2828 & 4157 & 5587 & 5443 & 1814 \\
\hline $1930-32$ & 32377 & 3319 & 1602 & 527 & 339 & 568 & 666 & 1508 & 1812 & 2731 & 4380 & 6630 & 6178 & 2117 \\
\hline $1940-42$ & 33715 & 2852 & 921 & 373 & 283 & 595 & 656 & 1382 & 1672 & 2528 & 4630 & 7674 & 7613 & 2536 \\
\hline $1980-82$ & 32326 & 337 & 49 & 37 & 44 & 74 & 73 & 213 & 493 & 1456 & 3565 & 7781 & 11333 & 6871 \\
\hline 1990 - 92 & 31747 & 190 & 45 & 20 & 29 & 49 & 72 & 218 & 458 & 1093 & 2966 & 6630 & 11079 & 8898 \\
\hline \multirow[t]{2}{*}{$2000-02$} & 30235 & 123 & 24 & 14 & 21 & 50 & 64 & 199 & 493 & 1110 & 2341 & 5326 & 9785 & 10685 \\
\hline & KIWA & KIWB & KIWC & KIWD & KIWE & KIWF & KIWG & $\mathrm{KIWH}$ & KIWI & KIWJ & KIWK & KIWL & KIWM & KIWN \\
\hline 1997 & 31189 & 130 & 23 & 28 & 21 & 43 & 71 & 199 & 496 & 1128 & 2480 & 5985 & 10164 & 10421 \\
\hline 1998 & 31032 & 137 & 26 & 28 & 19 & 55 & 68 & 198 & 485 & 1106 & 2416 & 5955 & 9913 & 10626 \\
\hline 1999 & 31676 & 115 & 26 & 20 & 17 & 65 & 58 & 201 & 467 & 1128 & 2431 & 5837 & 10198 & 11113 \\
\hline 2000 & 30288 & 132 & 20 & 10 & 21 & 46 & 56 & 222 & 510 & 1086 & 2324 & 5512 & 9875 & 10474 \\
\hline 2001 & 30058 & 135 & 20 & 16 & 21 & 47 & 71 & 189 & 480 & 1111 & 2361 & 5235 & 9695 & 10677 \\
\hline 2002 & 30360 & 103 & 32 & 15 & 20 & 58 & 65 & 185 & 489 & 1134 & 2339 & 5232 & 9784 & 10904 \\
\hline 2003 & 30640 & 119 & 24 & 18 & 20 & 57 & 64 & 181 & 489 & 1062 & 2446 & 5194 & 9977 & 10989 \\
\hline 2004 & 29412 & 106 & 19 & 15 & 22 & 52 & 62 & 179 & 492 & 1065 & 2291 & 4924 & 9924 & 10261 \\
\hline 2005 & 29225 & 125 & 27 & 11 & 18 & 55 & 58 & 163 & 506 & 1073 & 2316 & 4841 & 9620 & 10412 \\
\hline 2006 & 28842 & 103 & 26 & 11 & 17 & 40 & 58 & 170 & 497 & 1090 & 2351 & 4722 & 9303 & 10454 \\
\hline 2007 & 29091 & 118 & 26 & 11 & 14 & 50 & 52 & 166 & 490 & 1124 & 2311 & 4732 & 9083 & 10914 \\
\hline 2008 & 29196 & 113 & 17 & 11 & 18 & 55 & 68 & 172 & 497 & 1083 & 2315 & 4560 & 9299 & 10988 \\
\hline
\end{tabular}




\begin{tabular}{|c|c|c|c|c|c|c|c|c|c|c|c|c|c|c|}
\hline & $\begin{array}{c}\text { All } \\
\text { ages }^{2}\end{array}$ & $\begin{array}{l}\text { Under } \\
1 \text { year }\end{array}$ & $1-4$ & $5-9$ & $10-14$ & $15-19$ & $20-24$ & $25-34$ & $35-44$ & $45-54$ & $55-64$ & $65-74$ & $75-84$ & $\begin{array}{r}85 \text { and } \\
\text { over }\end{array}$ \\
\hline \multicolumn{15}{|c|}{ Northern Ireland } \\
\hline \multicolumn{15}{|l|}{ Males } \\
\hline $1900-02$ & 11554 & 1958 & 985 & 280 & 206 & 367 & 446 & 779 & 669 & 832 & 1413 & 1666 & 1368 & 585 \\
\hline $1910-12$ & 10469 & 1697 & 841 & 222 & 152 & 282 & 311 & 569 & 679 & 836 & 1148 & 2011 & 1287 & 434 \\
\hline $1920-22$ & 9622 & 1491 & 596 & 191 & 150 & 258 & 334 & 496 & 570 & 869 & 1159 & 1749 & 1324 & 435 \\
\hline $1940-42$ & 9383 & 1258 & 322 & 117 & 102 & 153 & 202 & 379 & 462 & 751 & 1290 & 2305 & 1637 & 405 \\
\hline $1950-52$ & 8197 & 658 & 105 & 55 & 36 & 50 & 77 & 179 & 318 & 671 & 1169 & 2117 & 2178 & 583 \\
\hline $1960-62$ & 8080 & 499 & 67 & 38 & 27 & 49 & 48 & 108 & 216 & 623 & 1419 & 2037 & 2131 & 816 \\
\hline $1970-72$ & 8786 & 396 & 58 & 40 & 32 & 76 & 83 & 115 & 221 & 643 & 1590 & 2493 & 2157 & 881 \\
\hline $1980-82$ & 8420 & 211 & 37 & 20 & 26 & 77 & 92 & 144 & 202 & 539 & 1414 & 2518 & 2346 & 795 \\
\hline $1990-92$ & 7550 & 102 & 21 & 13 & 18 & 55 & 73 & 132 & 178 & 423 & 1044 & 2175 & 2393 & 922 \\
\hline \multirow[t]{2}{*}{$2000-02$} & 7029 & 64 & 13 & 11 & 14 & 50 & 62 & 128 & 195 & 411 & 851 & 1656 & 2347 & 1228 \\
\hline & $\mathrm{KHXA}$ & KHXB & $\mathrm{KHXC}$ & $\mathrm{KHXD}$ & KHXE & KHXF & KHXG & $\mathrm{KHXH}$ & $\mathrm{KHXI}$ & KHXJ & KHXK & $\mathrm{KHXL}$ & KHXM & $\mathrm{KHXN}$ \\
\hline 1997 & 7244 & 68 & 12.0 & 11 & 12 & 50 & 62 & 122 & 172 & 411 & 924 & 1937 & 2367 & 1096 \\
\hline 1999 & 7464 & 82 & 20.0 & 16 & 11 & 45 & 68 & 124 & 194 & 409 & 876 & 1871 & 2456 & 1292 \\
\hline 2000 & 7128 & 61 & 12.0 & 12 & 14 & 55 & 69 & 130 & 191 & 387 & 869 & 1682 & 2426 & 1220 \\
\hline 2001 & 7007 & 79 & 7.0 & 11 & 14 & 48 & 63 & 119 & 189 & 423 & 785 & 1669 & 2370 & 1231 \\
\hline 2002 & 6948 & 52 & 19.0 & 9 & 14 & 48 & 54 & 134 & 206 & 421 & 898 & 1616 & 2245 & 1232 \\
\hline 2003 & 6920 & 74 & 11.0 & 10 & 14 & 39 & 52 & 94 & 197 & 361 & 817 & 1659 & 2310 & 1282 \\
\hline 2004 & 6935 & 64 & 13.0 & 11 & 7 & 37 & 46 & 101 & 205 & 417 & 914 & 1544 & 2366 & 1210 \\
\hline 2005 & 6957 & 81 & 9.0 & 9 & 10 & 60 & 82 & 129 & 210 & 429 & 875 & 1457 & 2289 & 1317 \\
\hline 2006 & 7062 & 70 & 15.0 & 8 & 17 & 52 & 64 & 119 & 232 & 453 & 894 & 1472 & 2267 & 1399 \\
\hline 2007 & 7208 & 70 & 15.0 & 5 & 13 & 55 & 66 & 112 & 251 & 467 & 914 & 1426 & 2368 & 1446 \\
\hline 2008 & 7227 & 63 & 13.0 & 12 & 12 & 70 & 58 & 127 & 232 & 436 & 832 & 1531 & 2278 & 1563 \\
\hline \multicolumn{15}{|l|}{ Females } \\
\hline $1900-02$ & 12735 & 1537 & 1013 & 317 & 277 & 495 & 521 & 1012 & 902 & 1075 & 1612 & 1876 & 1484 & 614 \\
\hline $1910-12$ & 11397 & 1369 & 808 & 249 & 218 & 325 & 365 & 785 & 811 & 961 & 1282 & 2328 & 1402 & 494 \\
\hline $1920-22$ & 10415 & 1149 & 547 & 193 & 188 & 299 & 331 & 687 & 713 & 939 & 1236 & 1976 & 1639 & 518 \\
\hline $1930-32$ & 9044 & 824 & 380 & 125 & 102 & 184 & 226 & 475 & 539 & 774 & 1293 & 2046 & 1541 & 535 \\
\hline $1940-42$ & 9229 & 910 & 305 & 105 & 90 & 159 & 174 & 409 & 490 & 688 & 1207 & 2246 & 1873 & 572 \\
\hline $1980-82$ & 7917 & 160 & 26 & 17 & 17 & 23 & 29 & 65 & 127 & 329 & 881 & 1813 & 2752 & 1678 \\
\hline $1990-92$ & 7620 & 80 & 20 & 12 & 9 & 16 & 17 & 53 & 117 & 267 & 672 & 1538 & 2636 & 2182 \\
\hline \multirow[t]{2}{*}{$2000-02$} & 7638 & 50 & 9 & 7 & 5 & 13 & 17 & 49 & 129 & 266 & 548 & 1263 & 2479 & 2802 \\
\hline & $\mathrm{KIXA}$ & $\mathrm{KIXB}$ & $\mathrm{KIXC}$ & KIXD & KIXE & KIXF & KIXG & $\mathrm{KIXH}$ & KIXI & KIXJ & KIXK & KIXL & KIXM & $\mathrm{KIXN}$ \\
\hline 1997 & 7727 & 69 & 13 & 10 & 9 & 18 & 13 & 43 & 90 & 268 & 570 & 1409 & 2604 & 2611 \\
\hline 1998 & 7672 & 60 & 12 & 8 & 7 & 24 & 20 & 49 & 126 & 258 & 531 & 1351 & 2509 & 2717 \\
\hline 1999 & 8199 & 66 & 12 & 7 & 8 & 23 & 25 & 55 & 119 & 273 & 583 & 1303 & 2765 & 2960 \\
\hline 2000 & 7775 & 48 & 11 & 11 & 3 & 19 & 13 & 51 & 125 & 283 & 574 & 1308 & 2526 & 2803 \\
\hline 2001 & 7506 & 55 & 7 & 4 & 8 & 14 & 14 & 41 & 137 & 260 & 510 & 1265 & 2404 & 2787 \\
\hline 2002 & 7638 & 48 & 8 & 6 & 5 & 7 & 24 & 56 & 124 & 258 & 561 & 1217 & 2507 & 2817 \\
\hline 2003 & 7542 & 41 & 7 & 5 & 8 & 14 & 14 & 52 & 133 & 254 & 527 & 1179 & 2511 & 2797 \\
\hline 2004 & 7419 & 58 & 9 & 5 & 6 & 18 & 16 & 50 & 139 & 259 & 560 & 1153 & 2447 & 2699 \\
\hline 2005 & 7267 & 59 & 7 & 8 & 9 & 16 & 21 & 40 & 121 & 244 & 542 & 1085 & 2409 & 2706 \\
\hline 2006 & 7470 & 51 & 10 & 5 & 15 & 16 & 18 & 52 & 135 & 286 & 556 & 1066 & 2364 & 2896 \\
\hline 2007 & 7441 & 53 & 5 & 5 & 6 & 16 & 17 & 49 & 121 & 291 & 549 & 1050 & 2328 & 2951 \\
\hline 2008 & 7680 & 60 & 9 & 10 & 3 & 24 & 18 & 50 & 138 & 306 & 558 & 1053 & 2333 & 3118 \\
\hline
\end{tabular}

1 See chapter text.

2 In some years the totals include a small number of persons whose age was

Sources: Office for National Statistics: 01329444410 General Register Office for Scotland; not stated. 
Population and vital statistics

$5.20 \begin{aligned} & \text { Infant and maternal mortality } \\ & \text { (i) - By country. (ii) - Infant mortality by country, type of death and sex }\end{aligned}$

\begin{tabular}{|c|c|c|c|c|c|c|c|c|c|c|c|c|c|c|c|c|}
\hline & \multicolumn{12}{|c|}{ Deaths of Infants under 1 year of age per thousand live births } & \multicolumn{4}{|c|}{$\begin{array}{l}\text { Maternal deaths per thousand } \\
\text { live births }{ }^{3}\end{array}$} \\
\hline & \multicolumn{3}{|c|}{ United Kingdom } & \multicolumn{3}{|c|}{ England and Wales ${ }^{2}$} & \multicolumn{3}{|c|}{ Scotland } & \multicolumn{3}{|c|}{ Northern Ireland } & \multirow{2}{*}{$\begin{array}{r}\text { United } \\
\text { Kingdom }\end{array}$} & \multirow{2}{*}{$\begin{array}{r}\text { England } \\
\text { and } \\
\text { Wales }\end{array}$} & \multirow[b]{2}{*}{ Scotland } & \multirow{2}{*}{$\begin{array}{l}\text { Northern } \\
\text { Ireland }\end{array}$} \\
\hline & Total & Males & Females & Total & Males & Females & Total & Males & Females & Total & Males & Females & & & & \\
\hline $1900-02$ & 142 & 156 & 128 & 146 & 160 & 131 & 124 & 136 & 111 & 113 & 123 & 103 & 4.71 & 4.67 & 4.74 & 6.03 \\
\hline $1910-12$ & 110 & 121 & 98 & 110 & 121 & 98 & 109 & 120 & 97 & 101 & 110 & 92 & 3.95 & 3.67 & 5.65 & 5.28 \\
\hline $1920-22$ & 82 & 92 & 71 & 80 & 90 & 69 & 94 & 106 & 82 & 86 & 95 & 77 & 4.37 & 4.03 & 6.36 & 5.62 \\
\hline 1930 - 32 & 67 & 75 & 58 & 64 & 72 & 55 & 84 & 94 & 73 & 75 & 83 & 66 & 4.54 & 4.24 & 6.40 & 5.24 \\
\hline $1940-42$ & 59 & 66 & 51 & 55 & 62 & 48 & 77 & 87 & 66 & 80 & 89 & 70 & 3.29 & 2.74 & 4.50 & 3.79 \\
\hline $1950-52$ & 30 & 34 & 26 & 29 & 33 & 25 & 37 & 42 & 32 & 40 & 45 & 36 & 0.88 & 0.79 & 1.09 & 1.09 \\
\hline $1960-62$ & 22 & 25 & 19 & 22 & 24 & 19 & 26 & 30 & 22 & 27 & 30 & 24 & 0.36 & 0.36 & 0.37 & 0.43 \\
\hline $1970-72$ & 18 & 20 & 16 & 18 & 20 & 15 & 19 & 22 & 17 & 22 & 24 & 20 & 0.17 & 0.17 & 0.17 & 0.12 \\
\hline 1980 - 82 & 12 & 13 & 10 & 11 & 13 & 10 & 12 & 13 & 10 & 13 & 15 & 12 & 0.09 & 0.09 & 0.14 & 0.06 \\
\hline $1990-92$ & 7 & 8 & 6 & 7 & 8 & 6 & 7 & 8 & 6 & 7 & 8 & 6 & 0.07 & 0.07 & 0.10 & \\
\hline $2000-02$ & 5 & 6 & 5 & 5 & 6 & 5 & 5 & 6 & 5 & 5 & 6 & 5 & 0.07 & 0.06 & 0.12 & 0.05 \\
\hline 1997 & 5.8 & 6.4 & 5.3 & 5.9 & 6.5 & 5.3 & 5.3 & 6.1 & 4.5 & 5.6 & 5.5 & 5.8 & 0.06 & 0.06 & 0.07 & - \\
\hline 1998 & 5.7 & 6.3 & 5.0 & 5.7 & 6.4 & 5.0 & 5.6 & 6.2 & 4.9 & 5.6 & 6.1 & 5.1 & 0.07 & 0.07 & 0.09 & 0.04 \\
\hline 1999 & 5.8 & 6.4 & 5.1 & 5.8 & 6.5 & 5.1 & 5.0 & 5.7 & 4.3 & 6.4 & 6.8 & 5.9 & 0.05 & 0.05 & 0.13 & - \\
\hline 2000 & 5.6 & 6.1 & 5.0 & 5.6 & 6.1 & 5.1 & 5.7 & 6.4 & 5.1 & 5.0 & 5.4 & 4.6 & 0.07 & 0.06 & 0.15 & - \\
\hline 2001 & 5.5 & 6.0 & 5.0 & 5.4 & 5.9 & 4.9 & 5.5 & 5.8 & 5.2 & 6.0 & 6.9 & 5.1 & 0.07 & 0.07 & 0.11 & 0.09 \\
\hline 2002 & 5.2 & 5.9 & 4.5 & 5.2 & 5.9 & 4.5 & 5.3 & 6.4 & 4.1 & 4.6 & 4.7 & 4.5 & 0.06 & 0.06 & 0.10 & 0.05 \\
\hline 2003 & 5.3 & 5.7 & 4.9 & 5.3 & 5.7 & 4.9 & 5.1 & 5.4 & 4.7 & 5.2 & 6.5 & 3.9 & 0.10 & 0.07 & 0.10 & 0.14 \\
\hline 2004 & 5.0 & 5.5 & 4.6 & 5.0 & 5.5 & 4.6 & 4.9 & 5.8 & 4.0 & 5.3 & 5.4 & 5.2 & 0.07 & 0.07 & 0.11 & 0.04 \\
\hline 2005 & 5.1 & 5.7 & 4.5 & 5.0 & 5.7 & 4.4 & 5.2 & 5.7 & 4.8 & 6.1 & 7.0 & 5.3 & 0.06 & 0.06 & 0.07 & 0.04 \\
\hline 2006 & 5.0 & 5.4 & 4.5 & 5.0 & 5.4 & 4.6 & 4.5 & 5.1 & 3.8 & 5.1 & 5.7 & 4.4 & 0.07 & 0.06 & 0.13 & 0.13 \\
\hline 2007 & 4.8 & 5.3 & 4.3 & 4.8 & 5.3 & 4.3 & 4.7 & 5.2 & 4.2 & 4.9 & 5.5 & 4.4 & 0.07 & 0.07 & 0.14 & - \\
\hline 2008 & 4.7 & 5.2 & 4.2 & 4.8 & 5.3 & 4.2 & 4.2 & 4.6 & 3.8 & 4.7 & 4.7 & 4.7 & 0.06 & 0.06 & 0.08 & - \\
\hline
\end{tabular}


Population and vital statistics

5.20 Infant and maternal mortality

(i) - By country. (ii) - Infant mortality by country, type of death and sex

continued

1998

1999

2000

2001

2002

2003

Deaths per thousand live births

Total

United Kingdom:

Stillbirths ${ }^{4}$

Perinatal

Neonatal

KHNQ

KHNR

KHNR
KHNS

$\begin{array}{ll}5.4 & 5.3 \\ 8.3 & 8.2\end{array}$

$\begin{array}{ll}3.8 & 3.9 \\ 1.9 & 1.9\end{array}$

5.3
8.1

5.3

$\begin{array}{ll}8.9 & 3.0 \\ 3.7 & 3.6\end{array}$

England and Wales:

Stillbirths ${ }^{4}$

Perinatal $^{4}$

Neonatal

Post neonatal

KHNU

KHNV

KHNW

$\begin{array}{ll}5.3 & 5.3 \\ 8.2 & 8.2\end{array}$

$3.8-3.9$

1.7

3.6
1.8

8.3

3.5
1.7

5.7
8.5
3.6

5.7
8.3

$\begin{array}{llll}5.3 & 5.3 & 5.2 & 5.1 \\ 8.0 & 7.9 & 7.7 & 7.5\end{array}$

Scotland:

Stillbirths ${ }^{4}$

Perinatal $^{4}$

Neonatal

Post neonatal

KHNY

KHNZ

KHOA

1.9

1.9

5.3
8.2
3.9
1.7

$\begin{array}{ll}5.3 & 5.6 \\ 8.0 & 8.3\end{array}$

$3.9 \quad 3.6$

1.9

$\begin{array}{lllll}6.1 & 5.2 & 5.6 & 5.7 & 5.4 \\ 8.7 & 7.6 & 8.4 & 8.5 & 7.6 \\ 3.6 & 3.3 & 4.0 & 3.8 & 3.2\end{array}$

Northern Ireland:

Stillbirths ${ }^{4}$

Perinatal $^{4}$

Neonatal

KHOC

Post neonatal

KHOD

KHOE

KHOF

$\begin{array}{ll}3.0 & 1.7\end{array}$

4.0
1.8

$\begin{array}{rrr}5.1 & 5.7 & 4.3 \\ 8.1 & 10.0 & 7.2 \\ 3.9 & 4.8 & 3.8\end{array}$

3.9
1.7

4.8
1.6

3.8
1.2

$\begin{array}{ll}5.1 & 5.7 \\ 8.4 & 8.7 \\ 4.4 & 3.4 \\ 1.6 & 1.2\end{array}$

Males

United Kingdom:

Perinatal ${ }^{4}$

Neonatal

Infant mortality

KHOG

$\mathrm{KHOH}$

KHOI

$8.8 \quad 8.7$

$\begin{array}{ll}8.7 & 8.7\end{array}$

$8.7 \quad 8.6$

8.6

$1.7 \quad 1.6$

$1.6 \quad 1.5$

$1.5 \quad 1.5$

England and Wales:

Perinatal ${ }^{4}$

KHOK

$\begin{array}{ll}\text { Neonatal } & \text { KHOL } \\ \text { Infant mortality } & \text { KHOM }\end{array}$

6.36.

4.2
6.1

6.0

$\begin{array}{lllll}8.8 & 8.6 & 8.7 & 8.5 & 8.9\end{array}$

$4.3 \quad 4.3$

$6.4 \quad 6.5$

$\begin{array}{ll}8.2 & 8.5 \\ 6.1 & 3.9\end{array}$

6.1

5.9

$\begin{array}{lllll}9.6 & 8.4 & 9.5 & 9.2 & 7.9\end{array}$

cotland:

Neonatal

KHOO

KHOP

$\begin{array}{ll}4.0 & 3.8 \\ 6.2 & 5.7\end{array}$

9.5
4.5

9.2
4.0
5.8

Infant mortality

6.2

5.7

6.4

$8.9 \quad 10.5$

$\begin{array}{lll}7.9 & 9.6 & 9.8\end{array}$

Perinatal $^{4}$

Neonatal

KHOS

KHOT

$\begin{array}{ll}4.4 & 5.5 \\ 6.1 & 6.8\end{array}$

4.2

$\begin{array}{ll}9.6 & 9.8 \\ 5.2 & 3.7\end{array}$

$\begin{array}{ll}5.7 & 5.7 \\ 8.5 & 8.4 \\ 3.6 & 3.5 \\ 1.7 & 1.6\end{array}$

$\begin{array}{llll}5.4 & 5.4 & 5.2 & 5.1\end{array}$

$\begin{array}{llll}8.0 & 8.0 & 7.7 & 7.6 \\ 3.4 & 3.5 & 3.3 & 3.2\end{array}$

$\begin{array}{llll}3.4 & 3.5 & 3.3 & 3.2 \\ 1.6 & 1.5 & 1.5 & 1.5\end{array}$

Infant mortality

KHOU

Females

United Kingdom:

Perinatal ${ }^{4}$

Neonatal

KHOW

KHOX

$\begin{array}{ll}7.7 & 7.8\end{array}$

Infant mortality

KHOY

$\begin{array}{ll}3.3 & 3.4 \\ 5.0 & 5.1\end{array}$

$\begin{array}{ll}7.5 & 7.4 \\ 3.5 & 3.3\end{array}$

$\begin{array}{ll}7.4 & 7.7\end{array}$

$\begin{array}{ll}8.2 \\ 3.1 & 8.2\end{array}$

$\begin{array}{llllll}7.9 & 7.6 & 7.5 & 7.3 & 7.1\end{array}$

England and Wales:

Perinatal $^{4}$

Neonatal

KHPA

$\begin{array}{ll}7.7 & 7.8\end{array}$

$7.6 \quad 7.3$

Infant mortality $\quad$ KHPC

$3.3 \quad 3.5$

7.6
3.5

$\begin{array}{ll}7.3 & 7.7\end{array}$

$\begin{array}{llllll}5.6 & 5.8 & 5.3 & 5.3 & 5.6 & 5.4 \\ 8.0 & 8.1 & 7.7 & 7.4 & 7.8 & 7.4 \\ 3.4 & 3.1 & 3.5 & 3.5 & 3.3 & 2.8 \\ 1.7 & 1.9 & 1.7 & 1.5 & 1.5 & 1.4\end{array}$

1.7

$\begin{array}{ll}1.9 & 1.7\end{array}$

3.5
1.5

$\begin{array}{ll}1.5 & 1.4\end{array}$

Scotland:

Perinatal

Neonatal

Infant mortality

KHPE

KHPF

$\begin{array}{lllll}7.9 & 6.7 & 7.2 & 7.8 & 7.2\end{array}$

5.0
8.0
3.9

$\begin{array}{lllll}5.0 & 4.0 & 3.8 & 4.2 & 4.5 \\ 8.0 & 8.1 & 6.9 & 6.9 & 7.4 \\ 3.6 & 4.9 & 3.8 & 3.2 & 3.6\end{array}$

$\begin{array}{lllll}3.0 & 8.1 & 6.9 & 6.9 & 7.4 \\ 1.7 & 1.3 & 3.8 & 3.2 & 3.6\end{array}$

Northern Ireland:

Perinatal ${ }^{4}$

Neonatal

Infant mortality

KHPG

$\begin{array}{ll}7.9 & 6.7 \\ 3.2 & 2.8\end{array}$

$\begin{array}{ll}7.2 & 7.8 \\ 3.5 & 3.5\end{array}$

$4.9 \quad 4.3$

5.1

3.5
5.2

7.2
2.6
4.1

$7.7 \quad 7.3$

$\begin{array}{lllll}7.3 & 7.9 & 7.1 & 7.3 & 6.7\end{array}$

See chapter text.

$\begin{array}{lllllll}\text { KHPI } & 7.3 & 9.5 & 6.4 & 6.9 & 7.6 & 7.8\end{array}$

KHPJ

KHPK

$\begin{array}{llllll}3.4 & 4.1 & 3.3 & 3.5 & 3.0 & 3.2 \\ 5.1 & 5.9 & 4.6 & 5.1 & 4.5 & 3.9\end{array}$

2 From 1937 to 1956 death rates are based on the births to which they relate

in the current and preceding years.

3 Deaths in pregnancy and childbirth.

4 Deaths per 1,000 live and stillbirths. See chapter introduction.

Sources: Office for National Statistics

General Register Office for Scotland; General Register Office (Northern Ireland) 
Population and vital statistics

\section{Death rates by sex and age ${ }^{1}$ \\ United Kingdom}

Rates per 1,000 population

\begin{tabular}{|c|c|c|c|c|c|c|c|c|c|c|c|c|c|}
\hline & $\begin{array}{r}\text { All } \\
\text { ages }\end{array}$ & $0-4$ & $5-9$ & $10-14$ & $15-19$ & $20-24$ & $25-34$ & $35-44$ & $45-54$ & $55-64$ & $65-74$ & $75-84$ & $\begin{array}{r}85 \text { and } \\
\text { over }\end{array}$ \\
\hline \multicolumn{14}{|l|}{ Males } \\
\hline $1910-12$ & 14.9 & 40.5 & 3.3 & 2.0 & 3.0 & 3.9 & 5.0 & 8.0 & 14.9 & 29.8 & 62.1 & 133.8 & 261.5 \\
\hline $1920-22$ & 13.5 & 33.4 & 2.9 & 1.8 & 2.9 & 3.9 & 4.5 & 6.9 & 11.9 & 25.3 & 57.8 & 131.8 & 259.1 \\
\hline $1930-32$ & 12.9 & 22.3 & 2.3 & 1.5 & 2.6 & 3.3 & 3.5 & 5.7 & 11.3 & 23.7 & 57.9 & 134.2 & 277.0 \\
\hline $1940-42$ & & & & & & & & & & & & & \\
\hline $1950-52$ & 12.6 & 7.7 & 0.7 & 0.5 & 0.9 & 1.4 & 1.6 & 3.0 & 8.5 & 23.2 & 55.2 & 127.6 & 272.0 \\
\hline $1960-62$ & 12.5 & 6.4 & 0.5 & 0.4 & 0.9 & 1.1 & 1.1 & 2.5 & 7.4 & 22.2 & 54.4 & 123.4 & 251.0 \\
\hline $1970-72$ & 12.4 & 4.6 & 0.4 & 0.4 & 0.9 & 1.0 & 1.0 & 2.4 & 7.3 & 20.9 & 52.9 & 116.3 & 246.1 \\
\hline $1980-82$ & 12.1 & 3.2 & 0.3 & 0.3 & 0.8 & 0.9 & 0.9 & 1.9 & 6.3 & 18.2 & 46.7 & 107.1 & 224.9 \\
\hline $1990-92$ & 11.2 & 2.0 & 0.2 & 0.2 & 0.7 & 0.9 & 1.0 & 1.8 & 4.6 & 14.2 & 38.6 & 93.0 & 201.4 \\
\hline \multirow[t]{2}{*}{$2000-02$} & 10.0 & 1.4 & 0.1 & 0.2 & 0.5 & 0.8 & 1.0 & 1.6 & 4.0 & 10.4 & 28.9 & 75.2 & 187.7 \\
\hline & $\mathrm{KHZA}$ & KHZB & $\mathrm{KHZC}$ & KHZD & KHZE & $\mathrm{KHZF}$ & KHZG & $\mathrm{KHZH}$ & KHZJ & KHZK & $\mathrm{KHZL}$ & KHZM & $\mathrm{KHZN}$ \\
\hline 1997 & 10.6 & 1.5 & 0.2 & 0.2 & 0.6 & 1.0 & 1.0 & 1.7 & 4.1 & 11.8 & 33.9 & 83.2 & 196.7 \\
\hline 1998 & 10.5 & 1.5 & 0.1 & 0.2 & 0.6 & 0.9 & 1.1 & 1.7 & 4.1 & 11.6 & 33.0 & 81.8 & 193.6 \\
\hline 1999 & 10.5 & 1.5 & 0.1 & 0.2 & 0.6 & 0.9 & 1.0 & 1.7 & 4.1 & 11.2 & 32.2 & 80.9 & 195.7 \\
\hline 2001 & 9.9 & 1.3 & 0.1 & 0.2 & 0.6 & 0.8 & 1.0 & 1.6 & 4.0 & 10.4 & 28.6 & 74.8 & 186.9 \\
\hline 2002 & 9.9 & 1.4 & 0.1 & 0.2 & 0.5 & 0.8 & 1.0 & 1.6 & 4.0 & 10.1 & 27.8 & 74.1 & 188.2 \\
\hline 2003 & 9.9 & 1.4 & 0.1 & 0.1 & 0.5 & 0.8 & 1.0 & 1.6 & 3.9 & 9.9 & 27.0 & 73.6 & 191.7 \\
\hline 2004 & 9.5 & 1.4 & 0.1 & 0.1 & 0.5 & 0.7 & 0.9 & 1.6 & 3.8 & 9.3 & 25.5 & 70.6 & 176.3 \\
\hline 2005 & 9.5 & 1.4 & 0.1 & 0.1 & 0.5 & 0.7 & 0.9 & 1.6 & 3.7 & 9.3 & 24.8 & 68.7 & 187.8 \\
\hline 2006 & 9.2 & 1.3 & 0.1 & 0.2 & 0.5 & 0.7 & 0.9 & 1.6 & 3.7 & 9.1 & 23.7 & 65.5 & 164.5 \\
\hline 2007 & 9.2 & 1.3 & 0.1 & 0.1 & 0.5 & 0.7 & 0.9 & 1.6 & 3.6 & 9.0 & 23.1 & 63.9 & 162.5 \\
\hline 2008 & 9.2 & 1.3 & 0.1 & 0.1 & 0.5 & 0.7 & 0.9 & 1.7 & 3.6 & 8.8 & 22.7 & 62.2 & 162.6 \\
\hline \multicolumn{14}{|l|}{ Females } \\
\hline $1900-02$ & 16.3 & 47.9 & 4.3 & 2.6 & 3.5 & 4.3 & 5.8 & 9.0 & 14.4 & 27.9 & 59.3 & 127.0 & 262.6 \\
\hline $1910-12$ & 13.3 & 34.0 & 3.3 & 2.1 & 2.9 & 3.4 & 4.4 & 6.7 & 11.5 & 23.1 & 50.7 & 113.7 & 234.0 \\
\hline $1920-22$ & 11.9 & 26.9 & 2.8 & 1.9 & 2.8 & 3.4 & 4.1 & 5.6 & 9.3 & 19.2 & 45.6 & 111.5 & 232.4 \\
\hline $1930-32$ & 11.5 & 17.7 & 2.1 & 1.5 & 2.4 & 2.9 & 3.3 & 4.6 & 8.3 & 17.6 & 43.7 & 110.1 & 246.3 \\
\hline $1940-42$ & & & & &.. & .. & .. & & .. & & & & \\
\hline $1950-52$ & 11.2 & 6.0 & 0.5 & 0.4 & 0.7 & 1.0 & 1.4 & 2.3 & 5.3 & 12.9 & 35.5 & 98.4 & 228.8 \\
\hline $1960-62$ & 11.2 & 4.9 & 0.3 & 0.3 & 0.4 & 0.5 & 0.8 & 1.8 & 4.5 & 11.0 & 30.8 & 87.3 & 218.5 \\
\hline \multirow[t]{2}{*}{$2000-02$} & 10.5 & 1.1 & 0.1 & 0.1 & 0.2 & 0.3 & 0.4 & 1.0 & 2.6 & 6.4 & 17.9 & 51.1 & 157.3 \\
\hline & $\mathrm{KHZO}$ & KHZP & $K H Z Q$ & KHZR & KHZS & $\mathrm{KHZT}$ & $\mathrm{KHZU}$ & $\mathrm{KHZV}$ & KHZW & $\mathrm{KHZX}$ & KHZY & $\mathrm{KHZZ}$ & $\mathrm{KHZI}$ \\
\hline 1997 & 11.0 & 1.2 & 0.1 & 0.1 & 0.3 & 0.3 & 0.4 & 1.1 & 2.7 & 7.1 & 20.5 & 55.2 & 160.3 \\
\hline 1998 & 11.0 & 1.2 & 0.1 & 0.1 & 0.3 & 0.3 & 0.4 & 1.0 & 2.7 & 7.0 & 20.2 & 54.4 & 159.8 \\
\hline 1999 & 11.0 & 1.2 & 0.1 & 0.1 & 0.3 & 0.3 & 0.4 & 1.0 & 2.7 & 6.9 & 19.6 & 54.2 & 163.7 \\
\hline 2000 & 10.5 & 1.1 & 0.1 & 0.1 & 0.3 & 0.3 & 0.5 & 1.0 & 2.7 & 6.6 & 18.5 & 51.6 & 155.8 \\
\hline 2001 & 10.4 & 1.1 & 0.1 & 0.1 & 0.2 & 0.3 & 0.4 & 1.0 & 2.6 & 6.4 & 17.8 & 50.8 & 155.8 \\
\hline 2002 & 10.5 & 1.0 & 0.1 & 0.1 & 0.2 & 0.3 & 0.4 & 1.0 & 2.6 & 6.2 & 17.4 & 51.0 & 160.3 \\
\hline 2003 & 10.6 & 1.2 & 0.1 & 0.1 & 0.2 & 0.3 & 0.4 & 1.0 & 2.6 & 6.1 & 17.1 & 51.8 & 166.4 \\
\hline 2004 & 10.0 & 1.1 & 0.1 & 0.1 & 0.2 & 0.3 & 0.4 & 1.0 & 2.4 & 5.8 & 16.2 & 49.3 & 155.2 \\
\hline 2005 & 10.0 & 1.1 & 0.1 & 0.1 & 0.2 & 0.3 & 0.4 & 0.9 & 2.5 & 5.9 & 15.8 & 48.2 & 160.1 \\
\hline 2006 & 9.6 & 1.1 & 0.1 & 0.1 & 0.2 & 0.3 & 0.4 & 0.9 & 2.4 & 5.7 & 15.2 & 46.4 & 144.9 \\
\hline 2007 & 9.7 & 1.1 & 0.1 & 0.1 & 0.2 & 0.3 & 0.4 & 0.9 & 2.3 & 5.7 & 14.9 & 45.6 & 144.8 \\
\hline 2008 & 9.7 & 1.1 & 0.1 & 0.1 & 0.2 & 0.3 & 0.4 & 1.0 & 2.3 & 5.7 & 14.6 & 45.0 & 146.6 \\
\hline
\end{tabular}

1 See chapter text.

Sources: Office for National Statistics; General Register Office for Scotland; 


\begin{tabular}{|c|c|c|c|c|c|c|c|c|}
\hline & \multicolumn{4}{|c|}{ United Kingdom } & \multicolumn{4}{|c|}{ England and Wales } \\
\hline & \multicolumn{2}{|c|}{ Males } & \multicolumn{2}{|c|}{ Females } & \multicolumn{2}{|c|}{ Males } & \multicolumn{2}{|c|}{ Females } \\
\hline & $I_{x}$ & $e^{0}{ }_{x}$ & $I_{x}$ & $e^{0} x$ & $I_{x}$ & $\mathrm{e}_{\mathrm{x}}^{0}$ & $I_{x}$ & $e^{0}{ }_{x}$ \\
\hline \multicolumn{9}{|l|}{$\operatorname{Age}(x)$} \\
\hline 0 years & 100000 & 77.4 & 100000 & 81.6 & 100000 & 77.7 & 100000 & 81.8 \\
\hline 5 years & 99373 & 72.9 & 99481 & 77.1 & 99370 & 73.2 & 99479 & 77.3 \\
\hline 10 years & 99314 & 67.9 & 99437 & 72.1 & 99312 & 68.2 & 99435 & 72.3 \\
\hline 15 years & 99246 & 63.0 & 99385 & 67.1 & 99246 & 63.3 & 99383 & 67.3 \\
\hline 20 years & 99011 & 58.1 & 99275 & 62.2 & 99027 & 58.4 & 99278 & 62.4 \\
\hline 25 years & 98663 & 53.3 & 99145 & 57.3 & 98703 & 53.6 & 99153 & 57.5 \\
\hline 30 years & 98262 & 48.5 & 98979 & 52.4 & 98324 & 48.8 & 98991 & 52.6 \\
\hline 35 years & 97740 & 43.8 & 98739 & 47.5 & 97833 & 44.0 & 98759 & 47.7 \\
\hline 40 years & 97069 & 39.1 & 98378 & 42.7 & 97196 & 39.3 & 98412 & 42.8 \\
\hline 45 years & 96157 & 34.4 & 97810 & 37.9 & 96325 & 34.6 & 97856 & 38.1 \\
\hline 55 years & 92677 & 25.5 & 95484 & 28.7 & 92962 & 25.7 & 95596 & 28.8 \\
\hline 60 years & 89489 & 21.3 & 93373 & 24.3 & 89852 & 21.5 & 93537 & 24.4 \\
\hline 65 years & 84583 & 17.4 & 90117 & 20.0 & 85077 & 17.5 & 90362 & 20.2 \\
\hline 70 years & 77232 & 13.8 & 85149 & 16.1 & 77865 & 13.9 & 85532 & 16.2 \\
\hline 75 years & 66692 & 10.5 & 77413 & 12.4 & 66477 & 10.6 & 77951 & 12.5 \\
\hline 80 years & 51826 & 7.8 & 65356 & 9.2 & 52668 & 7.9 & 65040 & 9.2 \\
\hline 85 years & 32514 & 5.7 & 47007 & 6.6 & 34258 & 5.7 & 48733 & 6.6 \\
\hline \multirow[t]{4}{*}{90 years } & 16658 & 4.0 & 27849 & 4.5 & 17169 & 4.0 & 28475 & 4.5 \\
\hline & \multicolumn{4}{|c|}{ Scotland } & \multicolumn{4}{|c|}{ Northern Ireland } \\
\hline & \multicolumn{2}{|c|}{ Males } & \multicolumn{2}{|c|}{ Females } & \multicolumn{2}{|c|}{ Males } & \multicolumn{2}{|c|}{ Females } \\
\hline & $I_{x}$ & $e^{0}{ }_{x}$ & $I_{x}$ & $e^{0}{ }_{x}$ & $I_{x}$ & $e_{x}^{0}$ & $I_{x}$ & $e_{x}^{0}$ \\
\hline \multicolumn{9}{|l|}{$\operatorname{Age}(x)$} \\
\hline 0 years & 100000 & 75.0 & 100000 & 79.9 & 100000 & 76.3 & 100000 & 81.2 \\
\hline 5 years & 99423 & 70.4 & 99519 & 75.3 & 99338 & 71.8 & 99466 & 76.6 \\
\hline 20 years & 98955 & 55.7 & 99273 & 60.4 & 98715 & 57.2 & 99193 & 61.8 \\
\hline 25 years & 98403 & 51.0 & 99101 & 55.5 & 98254 & 52.5 & 99056 & 56.9 \\
\hline 30 years & 97799 & 46.3 & 98893 & 50.6 & 97740 & 47.7 & 98890 & 52.0 \\
\hline 35 years & 96910 & 41.7 & 98572 & 45.8 & 97239 & 43.0 & 98631 & 47.1 \\
\hline 40 years & 95889 & 37.1 & 98081 & 41.0 & 96496 & 38.3 & 98225 & 42.3 \\
\hline 45 years & 94608 & 32.6 & 97385 & 36.3 & 95430 & 33.7 & 97654 & 37.5 \\
\hline 50 years & 92821 & 28.1 & 96256 & 31.7 & 93817 & 29.2 & 96662 & 32.9 \\
\hline 55 years & 90118 & 23.9 & 94513 & 27.2 & 91575 & 24.9 & 95102 & 28.4 \\
\hline 60 years & 86187 & 19.9 & 91933 & 22.9 & 88394 & 20.7 & 92859 & 24.0 \\
\hline 65 years & 80137 & 16.2 & 87839 & 18.8 & 82994 & 16.8 & 89669 & 19.8 \\
\hline 70 years & 71517 & 12.8 & 81603 & 15.1 & 75558 & 13.2 & 84762 & 15.7 \\
\hline 75 years & 59691 & 9.8 & 72542 & 11.6 & 64767 & 10.0 & 76934 & 12.1 \\
\hline 80 years & 43431 & 7.3 & 59215 & 8.6 & 49454 & 7.3 & 64738 & 8.9 \\
\hline 85 years & 27194 & 5.4 & 41475 & 6.2 & 30645 & 5.2 & 47390 & 6.1 \\
\hline 90 years & 12522 & 3.9 & 22504 & 4.4 & 13759 & 3.7 & 25924 & 4.1 \\
\hline
\end{tabular}

Note Column $\mathrm{I}_{\mathrm{x}}$ shows the number who would survive to exact age $(\mathrm{x})$, out Source: Office for National Statistics: 01329444681 of 100,000 born, who were subject throughout their lives to the death rates experienced in the three-year period indicated. Column $e^{0}$ is 'the expectation of life', that is, the average future lifetime which would be lived by a person aged exactly $\mathrm{x}$ if likewise subject to the death rates experienced in the three-year period indicated. See introductory notes. 
5. 23 Adoptions by date of entry in Adopted Children Register: by sex, age and country

Numbers and Percentages

All ages

Under 1

$10-14$

$15-17$

Numbers Percentages Numbers Percentages Numbers Percentages Numbers Percentages Numbers Percentages Numbers Percentages

United Kingdom

Persons

$\begin{array}{rrrr} & \text { VOXI } & \text { VOXJ } & \text { VOXK } \\ 2002 & 6239 & 100 & 313 \\ 2003 & 5426 & 100 & 212 \\ 2004 & 6116 & 100 & 274 \\ 2005 & 6151 & 100 & 242 \\ 2006 & 5539 & 100 & 216 \\ 2007 & 5224 & 100 & 163\end{array}$

$\begin{array}{rr}\text { VOXL } & \text { VOXM } \\ 5 & 2737 \\ 4 & 2481 \\ 5 & 2843 \\ 4 & 3127 \\ 4 & 2788 \\ 3 & 2761\end{array}$

$\begin{array}{rr}\text { VOXN } & \text { VOXO } \\ 44 & 1937 \\ 46 & 1716 \\ 46 & 1856 \\ 51 & 1757 \\ 50 & 1608 \\ 53 & 1400\end{array}$

$\begin{array}{rr}\text { VOXP } & \text { VOXQ } \\ 31 & 999 \\ 32 & 789 \\ 30 & 847 \\ 29 & 798 \\ 29 & 696 \\ & \\ 27 & 672\end{array}$

$\begin{array}{rr}\text { VOXR } & \text { VOXS } \\ 16 & 253 \\ 15 & 228 \\ 14 & 269 \\ 13 & 228 \\ 13 & 226 \\ 13 & 205\end{array}$

VOXT

4

Males

$\begin{array}{ll} & \text { VOXU } \\ 2002 & 3140 \\ 2003 & 2634 \\ 2004 & 3051 \\ 2005 & 3072 \\ 2006 & 2708\end{array}$

$\begin{array}{rr}\text { VOXV } & \text { VOXW } \\ 100 & 176 \\ 100 & 104 \\ 100 & 145 \\ 100 & 121 \\ 100 & 97 \\ & \\ 100 & 83\end{array}$

$\begin{array}{rr}\text { VOXX } & \text { VOXY } \\ 6 & 1425 \\ 4 & 1224 \\ 5 & 1426 \\ 4 & 1566 \\ 4 & 1377 \\ & \\ 3 & 1392\end{array}$

$\begin{array}{rr}\text { VOXZ } & \text { VOYA } \\ 45 & 935 \\ 46 & 844 \\ 47 & 936 \\ 51 & 910 \\ 51 & 806 \\ & \\ 53 & 714\end{array}$

$\begin{array}{rr}\text { VOYB } & \text { VOYC } \\ 30 & 488 \\ 32 & 351 \\ 31 & 418 \\ 30 & 370 \\ 30 & 314 \\ & \\ 27 & 314\end{array}$

$\begin{array}{rr}\text { VOYD } & \text { VOYE } \\ 16 & 116 \\ 13 & 111 \\ 14 & 126 \\ 12 & 105 \\ 12 & 113 \\ 12 & 103\end{array}$

VOYF

$2007 \quad 2614$

83

$\begin{array}{rr}\text { VOYH } & \text { VOYI } \\ 100 & 137 \\ 100 & 108 \\ 100 & 129 \\ 100 & 121 \\ 100 & 119 \\ 100 & 80\end{array}$

$\begin{array}{rr}\text { VOYJ } & \text { VOYK } \\ 4 & 1312 \\ 4 & 1257 \\ 4 & 1417 \\ 4 & 1561 \\ 4 & 1411 \\ 3 & 1369\end{array}$

$\begin{array}{rr}\text { VOYL } & \text { VOYM } \\ 42 & 1002 \\ 45 & 872 \\ 46 & 920 \\ 51 & 847 \\ 50 & 802 \\ 52 & 686\end{array}$

$\begin{array}{rr}\text { VOYN } & \text { VOYO } \\ 32 & 511 \\ 31 & 438 \\ 30 & 456 \\ 28 & 428 \\ 28 & 382 \\ & \\ 26 & 358\end{array}$

$\begin{array}{rr}\text { VOYP } & \text { VOYQ } \\ 16 & 137 \\ 16 & 117 \\ 15 & 143 \\ 14 & 122 \\ 13 & 113 \\ 14 & 102\end{array}$

VOYR

4

England and Wales ${ }^{1}$

Persons

$\begin{array}{rrrr} & \text { GQTP } & \text { GQTQ } & \text { GQTR } \\ 2002 & 5680 & 100 & 287 \\ 2003 & 4818 & 100 & 183 \\ 2004 & 5562 & 100 & 253 \\ 2005 & 5565 & 100 & 22 \\ 2006 & 4980 & 100 & 197 \\ 2007 & 4637 & 100 & 15\end{array}$

$\begin{array}{rr}\text { GQTS } & \text { GQTT } \\ 5 & 2532 \\ 4 & 2260 \\ 5 & 2627 \\ 4 & 2906 \\ 4 & 2592 \\ 3 & 2510\end{array}$

$\begin{array}{rr}\text { GQTU } & \text { GQTV } \\ 45 & 1748 \\ 47 & 1503 \\ 47 & 1651 \\ 52 & 1555 \\ 52 & 1406 \\ & \\ 54 & 1206\end{array}$

$\begin{array}{rr}\text { GQTW } & \text { GQTX } \\ 31 & 900 \\ 31 & 683 \\ 30 & 786 \\ 28 & 683 \\ 28 & 585 \\ & \\ 26 & 576\end{array}$

$\begin{array}{rr}\text { GQTY } & \text { GQTZ } \\ 16 & 213 \\ 14 & 189 \\ 14 & 245 \\ 12 & 199 \\ 12 & 195 \\ 12 & 171\end{array}$

GQUA

4
4
4
4
4
4

Males

$\begin{array}{lr} & \text { GQUB } \\ 2002 & 2871 \\ 2003 & 2339 \\ 2004 & 2777 \\ 2005 & 2791 \\ 2006 & 2446 \\ 2007 & 2315\end{array}$

$\begin{array}{rr}\text { GQUC } & \text { GQUD } \\ 100 & 160 \\ 100 & 91 \\ 100 & 132 \\ 100 & 112 \\ 100 & 95 \\ 100 & 77\end{array}$

$\begin{array}{rr}\text { GQUE } & \text { GQUF } \\ 6 & 1324 \\ 4 & 1115 \\ 5 & 1327 \\ 4 & 1461 \\ 4 & 1282 \\ 3 & 1253\end{array}$

$\begin{array}{rr}\text { GQUG } & \text { GQUH } \\ 46 & 846 \\ 48 & 737 \\ 48 & 831 \\ 52 & 808 \\ 52 & 707 \\ & \\ 54 & 618\end{array}$

$\begin{array}{rr}\text { GQUI } & \text { GQUJ } \\ 29 & 443 \\ 32 & 301 \\ 30 & 373 \\ 29 & 320 \\ 29 & 267 \\ 27 & 270\end{array}$

$\begin{array}{rr}\text { GQUK } & \text { GQUL } \\ 15 & 98 \\ 13 & 95 \\ 13 & 114 \\ 11 & 90 \\ 11 & 94 \\ 12 & 89\end{array}$

GQUM

3
4
4
3
4
4

Females

\begin{tabular}{|c|c|c|c|c|c|c|c|c|c|c|c|c|}
\hline & GQUN & GQUO & GQUP & GQUQ & GQUR & GQUS & GQUT & GQUU & GQUV & GQUW & GQUX & GQUY \\
\hline 2002 & 2809 & 100 & 127 & 5 & 1208 & 43 & 902 & 32 & 457 & 16 & 115 & 4 \\
\hline 2003 & 2479 & 100 & 92 & 4 & 1145 & 46 & 766 & 31 & 382 & 15 & 94 & 4 \\
\hline 2004 & 2785 & 100 & 121 & 4 & 1300 & 47 & 820 & 29 & 413 & 15 & 131 & 5 \\
\hline 2005 & 2774 & 100 & 110 & 4 & 1445 & 52 & 747 & 27 & 363 & 13 & 109 & 4 \\
\hline 2006 & 2534 & 100 & 102 & 4 & 1310 & 52 & 699 & 28 & 318 & 13 & 101 & 4 \\
\hline 2007 & 2322 & 100 & 74 & 3 & 1257 & 54 & 588 & 25 & 306 & 13 & 82 & 4 \\
\hline
\end{tabular}


Population and vital statistics

Numbers Percentages Numbers Percentages Numbers Percentages Numbers Percentages Numbers Percentages Numbers Percentages

Scotland ${ }^{1}$

Persons

$\begin{array}{rrrr} & \text { GQUZ } & \text { GQVA } & \text { GQVB } \\ 2002 & 385 & 100 & 13 \\ 2003 & 468 & 100 & 25 \\ 2004 & 393 & 100 & 21 \\ 2005 & 439 & 100 & 18 \\ 2006 & 418 & 100 & 16 \\ & & & \end{array}$

$\begin{array}{rr}\text { GQVC } & \text { GQVD } \\ 3 & 143 \\ 5 & 153 \\ 5 & 144 \\ 4 & 162 \\ 4 & 153\end{array}$

$\begin{array}{rr}\text { GQVE } & \text { GQVF } \\ 37 & 130 \\ 33 & 170 \\ 37 & 143 \\ 37 & 155 \\ 37 & 150\end{array}$

$\begin{array}{rr}\text { GQVG } & \text { GQVH } \\ 34 & 73 \\ 36 & 88 \\ 36 & 67 \\ 35 & 81 \\ 36 & 73\end{array}$

$\begin{array}{rr}\text { GQVI } & \text { GQVJ } \\ 19 & 26 \\ 19 & 32 \\ 17 & 18 \\ 18 & 23 \\ 17 & 26\end{array}$

GQVK

32

65

$15 \quad 27$

Males

$\begin{array}{rrrr} & \text { GQVL } & \text { GQVM } & \text { GQVN } \\ 2002 & 193 & 100 & 8 \\ 2003 & 228 & 100 & 11 \\ 2004 & 200 & 100 & 13 \\ 2005 & 217 & 100 & 9 \\ 2006 & 194 & 100 & 2\end{array}$

\begin{tabular}{rr} 
GQVO & GQVP \\
4 & 75 \\
5 & 78 \\
7 & 67 \\
4 & 80 \\
1 & 72 \\
& \\
\hline
\end{tabular}

$\begin{array}{rr}\text { GQVQ } & \text { GQVR } \\ 39 & 60 \\ 34 & 85 \\ 34 & 77 \\ 37 & 79 \\ 37 & 78 \\ & \\ 50 & 70\end{array}$

$\begin{array}{rr}\text { GQVS } & \text { GQVT } \\ 31 & 37 \\ 37 & 43 \\ 39 & 34 \\ 36 & 36 \\ 40 & 25\end{array}$

13
11
9
13
17

GQVW

2007

229

100

Females

$\begin{array}{rrrr} & \text { GQVX } & \text { GQVY } & \text { GQV } \\ 2002 & 192 & 100 & \\ 2003 & 240 & 100 & 1 \\ 2004 & 193 & 100 & \\ 2005 & 222 & 100 & \\ 2006 & 224 & 100 & 14 \\ 2007 & 211 & 100 & \end{array}$

$\begin{array}{rr}\text { GQWA } & \text { GRFK } \\ 3 & 68 \\ 6 & 75 \\ 4 & 77 \\ 4 & 82 \\ 6 & 81\end{array}$

$\begin{array}{rr}\text { GRFL } & \text { GRFM } \\ 35 & 70 \\ 31 & 85 \\ 40 & 66 \\ 37 & 76 \\ 36 & 72 \\ 39 & 71\end{array}$

$\begin{array}{rr}\text { GRFN } & \text { GRFO } \\ 36 & 36 \\ 35 & 45 \\ 34 & 33 \\ 34 & 45 \\ 32 & 48 \\ 34 & 35\end{array}$

$\begin{array}{rr}\text { GRFP } & \text { GRFQ } \\ 19 & 13 \\ 19 & 21 \\ 17 & 9 \\ 20 & 10 \\ 22 & 9 \\ 17 & 17\end{array}$

GRFR

$\begin{array}{rr} & \text { GRFR } \\ 13 & 7 \\ 21 & 9 \\ 9 & 5 \\ 10 & 5 \\ 9 & 4\end{array}$

Northern Ireland ${ }^{1}$ Persons

$\begin{array}{rrr} & \text { VOYS } & \text { VOY } \\ 2003 & 140 & 100 \\ 2004 & 161 & 100 \\ 2005 & 140 & 100 \\ 2006 & 141 & 100 \\ 2007 & 147 & 100 \\ 2008 & 97 & 100\end{array}$

$\begin{array}{rrr}\text { VOYU } & \text { VOYV } & \text { VOYW } \\ 4 & 3 & 68 \\ 4 & 2 & 70 \\ 6 & 4 & 53 \\ 3 & 2 & 44 \\ 3 & 2 & 53\end{array}$

$\begin{array}{rrrr}\text { VOYX } & \text { VOYY } & \text { VOYZ } & \text { VOZA } \\ 49 & 43 & 31 & 18 \\ 43 & 61 & 38 & 20 \\ 38 & 45 & 32 & 30 \\ 31 & 51 & 36 & 38 \\ 36 & 53 & 36 & 31 \\ 49 & 30 & 31 & 13\end{array}$

$\begin{array}{rrr}\text { VOZB } & \text { VOZC } & \text { VOZD } \\ 13 & 7 & 5 \\ 12 & 6 & 4 \\ 21 & 6 & 4 \\ 27 & 5 & 4 \\ 21 & 7 & 5 \\ 13 & 6 & 6\end{array}$

Males

$\begin{array}{rrr} & \text { VOZE } & \text { VOZ } \\ 2003 & 67 & 100 \\ 2004 & 74 & 100 \\ 2005 & 61 & 100 \\ 2006 & 68 & 100 \\ 2007 & 70 & 100 \\ 2008 & 48 & 100\end{array}$

$\begin{array}{rrr}\text { VOZG } & \text { VOZH } & \text { VOZI } \\ 2 & 3 & 3 \\ 1 & 1 & 32 \\ 2 & 3 & 23 \\ - & - & 24 \\ 2 & 3 & 24\end{array}$

$\begin{array}{rr}\text { VOZJ } & \text { VOZK } \\ 46 & 22 \\ 43 & 28 \\ 38 & 21 \\ 35 & 20 \\ 34 & 26\end{array}$

$\begin{array}{rr}\text { VOZL } & \text { VOZM } \\ 33 & 7 \\ 38 & 10 \\ 34 & 12 \\ 29 & 22 \\ 37 & 14\end{array}$

$\begin{array}{rr}\text { VOZN } & \text { VOZO } \\ 10 & 5 \\ 14 & 3 \\ 20 & 3 \\ 32 & 2 \\ 20 & 4 \\ & \\ 13 & 5\end{array}$

VOZP

Females

\begin{tabular}{|c|c|c|c|c|c|c|c|c|c|c|c|c|}
\hline & VOZQ & VOZR & VOZS & VOZT & VOZU & VOZV & VOZW & VOZX & VOZY & VOZZ & VPAA & VPVD \\
\hline 2003 & 73 & 100 & 2 & 3 & 37 & 51 & 21 & 29 & 11 & 15 & 2 & 3 \\
\hline 2004 & 87 & 100 & 3 & 3 & 38 & 44 & 33 & 38 & 10 & 11 & 3 & 3 \\
\hline 2005 & 79 & 100 & 4 & 5 & 30 & 38 & 24 & 30 & 18 & 23 & 3 & 4 \\
\hline 2006 & 73 & 100 & 3 & 4 & 20 & 27 & 31 & 42 & 16 & 22 & 3 & 4 \\
\hline 2007 & 77 & 100 & 1 & 1 & 29 & 38 & 27 & 35 & 17 & 22 & 3 & 4 \\
\hline 2008 & 49 & 100 & - & - & 28 & 57 & 13 & 27 & 7 & 14 & 1 & 2 \\
\hline
\end{tabular}

\begin{tabular}{|c|c|c|c|c|c|c|}
\hline \multicolumn{3}{|c|}{1 England \& Wales: number of persons aged over 17 (counted into 'All ages') } & \multicolumn{4}{|c|}{ Northern Ireland: number of persons aged over 17 (counted into 15-17 } \\
\hline Year & Persons & Male Female & age & up) & & \\
\hline 2006 & 5 & 1 & Year & Persons & Male & Female \\
\hline 2007 & 23 & 15 & 2007 & 1 & 1 & \\
\hline \multicolumn{7}{|c|}{ Scotland: number of persons aged over 17 (counted into $15-17$ age group) } \\
\hline 2003 & 4 & 3 & & & \multirow{3}{*}{\multicolumn{2}{|c|}{$\begin{array}{r}\text { Sources: Office for National Statistics: } 01329444410 ; \\
\text { General Register Office for Scotland; } \\
\text { Northern Ireland Statistics and Research Agency }\end{array}$}} \\
\hline 2004 & 3 & 3 & & & & \\
\hline 2006 & 5 & 5 & & & & \\
\hline
\end{tabular}

